SHEP-12-18

November 5, 2018

\title{
Exploring Drell-Yan signals from the 4D Composite Higgs Model at the LHC
}

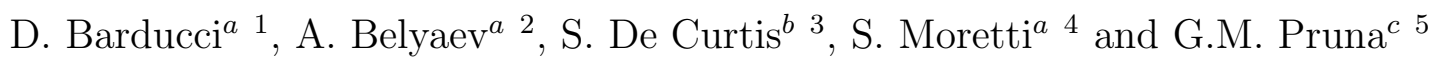 \\ a School of Physics and Astronomy, University of Southampton, \\ Southampton SO17 1BJ, U.K. \\ ${ }^{b}$ INFN, Sezione di Firenze, \\ Via G. Sansone 1, 50019 Sesto Fiorentino, Italy \\ ${ }^{c}$ TU Dresden, Institut ur Kern- und Teilchenphysik, \\ Zellescher Weg 19, D-01069 Dresden, Germany
}

\begin{abstract}
We study the phenomenology of Drell-Yan processes at the Large Hadron Collider for the case of both the neutral and charged current channels within a recently proposed 4-Dimensional formulation of the Minimal Composite Higgs Model. We estimate the integrated and differential event rates at the CERN machine, assuming $14 \mathrm{TeV}$ and data samples of $\mathcal{O}\left(100 \mathrm{fb}^{-1}\right)$, as at lower energy and/or luminosity event rates are prohibitively small. We pay particular attention to the presence of multiple resonances in either channel, by showing that in certain region of parameter space some of these can be distinguishable and experimentally accessible in the invariant and/or transverse mass distribution, sampled in either the cross section, the forward-backward asymmetry or both. At the same time, we assess the indirect impact onto the line-shape of the emerging gauge boson resonances, both neutral and charged, of additional heavy fermionic states present in the spectrum of the model. Finally, we show how to exploit in the kinematic selection the fact that the extra neutral and charged gauge boson resonances in composite Higgs models are correlated in mass. Such results rely on a parton level study including a statistical error analysis.
\end{abstract}

\footnotetext{
${ }^{1}$ E-mail: d.barducci@soton.ac.uk

${ }^{2}$ E-mail: a.belyaev@soton.ac.uk

${ }^{3}$ E-mail: decurtis@fi.infn.it

${ }^{4}$ E-mail: s.moretti@soton.ac.uk

${ }^{5}$ E-mail: giovanni_marco.pruna@tu-dresden.de
} 


\section{Introduction}

The Drell-Yan (DY) mechanism is one of the most important probes in the search for new vector boson resonances associated to possible physics Beyond the Standard Model (BSM). It consists of di-lepton production from hadron-hadron scattering via Neutral Current (NC) or Charged Current (CC) processes. From the theoretical point of view, such a mechanism is well under control as higher order effects from both Electro-Weak (EW) interactions and Quantum Chromo-Dynamics (QCD) are known up to one- and two-loop contributions, respectively (see, e.g., Ref. [1] for a review). From the experimental point of view, the directions and energies of the particles of such final state are well reconstructed in a generic detector at an hadron machine, especially when consisting of electrons or muons ( $e$ or $\mu$ ) and/or even their related neutrinos. For all such reasons, it is clear that this class of processes is ideal also for identifying the mass of the intermediate bosons being produced and studying their properties.

Nowadays, the Large Hadron Collider (LHC) offers an unprecedented chance to test DY phenomenology in high energy proton-proton scattering. Therefore, we currently have the opportunity to test many choices of models with additional vector bosons (generically denoted as $Z^{\prime}$ and $W^{\prime}$ ) in the particle spectrum, being their phenomenology of primary interest provided that the new gauge sector is considerably coupled to the SM fermions. Concerning this kind of models, the community is showing a growing interest to bottomup approaches to extra $Z^{\prime}$ and $W^{\prime}$ models (see [2]) as well as to top-down schemes (e.g., exploiting Supersymmetry [3, 4], little Higgs inspired [5], based on extra-dimensions [6, 7, 8], etc.).

In this paper, we adopt the latter approach and focus on the recently proposed 4Dimensional Composite Higgs Model (4DCHM) of Ref. 9]. Amongst the many alternative (to the SM) EW Symmetry Breaking (EWSB) scenarios proposed over the years, the one with a Higgs as a pseudo Nambu Goldstone Boson (PNGB) associated to the spontaneous breaking of a symmetry $G$ to $H$ can perhaps give one of the most natural solutions to the hierarchy problem of the SM. The idea goes back to the '80s [10]. However, one modern ingredient is the mechanism of 'partial compositness', wherein (some of) the SM gauge fields and fermions mix with new force and matter states emerging such an alternative EWSB. The simplest example, based on the symmetry pattern $S O(5) / S O(4)$, was considered in [7] in the context of Randall-Sundrum scenarios. The $4 \mathrm{D}$ effective description of this scenario, proposed in [9], is a highly deconstructed version of the 5D theory and presents several exceptional features. In all generality, an extreme deconstruction of 5D composite Higgs models with partial compositness leads to just two 4D sectors: the elementary one, that contains the SM structure $S U(2)_{L} \otimes U(1)_{Y}$, and the composite one, which include the extra (bosonic and fermionic) resonances. They are mixed in order to realise the partial compositness mechanism and EWSB is induced by means of a composite 4D Higgs state which is a PNGB stemming from the latter. From the phenomenological point of view, the only degrees of freedom which might be accessible at the LHC are represented by the lowest lying resonances described by the extreme deconstruction in the $4 \mathrm{DCHM}$ of Ref. 9]. Here a minimal choice for the fermionic sector is assumed, as one includes two $S O(5)$ multiplets of resonances per family in the composite sector. This is indeed the minimal matter content that allows for a finite Higgs potential calculable via the Coleman-Weinberg technique. From the explicit expression of the 
Higgs potential one can extract the Higgs Vacuum Expectation Value (VEV) and mass in terms of the model parameters. The peculiarity of this model is that, for a natural choice of the free parameters, both in the gauge and fermion sectors, the Higgs mass value that one obtains is compatibile with the most recent LHC results by the ATLAS [11] and CMS [12] experiments.

As intimated, in the composite sector of the 4DCHM, besides the Higgs state and extra heavy fermions (with both standard and exotic quantum numbers), one also has extra composite spin-1 resonances associated with the $S O(5) \otimes U(1)_{X}$ local symmetry: five $Z^{\prime}$ states and three $W^{\prime}$ states. These objects are weakly yet sizably coupled to the first and second generations of fermionic matter and this makes the 4DCHM an excellent candidate for a phenomenological analysis of DY processes at the LHC.

For the sake of completeness, we also remark that analogous EWSB scenarios have been already studied with respect to DY processes (see, e.g., Refs. [13] and [14], for the $\mathrm{NC}$ and $\mathrm{CC}$ case, respectively). However, the purpose of this paper is to surpass previous literature along four main directions:

1. we study the effects of the opening of fermionic decay channels onto the line shape of the DY resonances, then we prove that this allows us to extrapolate information on the mass and coupling spectrum of the fermionic sector of the model;

2. we consider the case of nearly degenerate gauge boson resonances and show that two of these can be resolved (albeit limited to the $\mathrm{NC}$ channel) over a large portion of the 4DCHM parameter space where decays of the additional gauge bosons into pairs of heavy fermions are forbidden;

3. we emphasise the role of the di-lepton invariant mass in investigating the above phenomenology not only in the usual terms of the cross section distribution but also in terms of the Forward-Backward Asymmetry (AFB) one;

4. we exploit correlated searches in the $\mathrm{CC}$ and $\mathrm{NC}$ channels and we show that kinematic information can be used to improve the overall event selection, as the mass of the additional neutral and charged gauge bosons of the 4DCHM are correlated by the underlying gauge symmetry.

The paper is structured as follows: in the next Section we describe the model, in Section 3 we illustrate its implementation in the numerical tools used for the analysis, in Section 4 we study the parameter space of the model and we select some benchmark points, in Section 5 we present our results and in Section 6 we state our conclusions.

\section{The $4 \mathrm{DCHM}$}

Let us recall the main characteristics of the 4DCHM introduced in [9], to which we refer for further details througout this section. The 4DCHM is an effective low-energy Lagrangian approximation of the deconstructed Minimal Composite Higgs Model (MCHM) of [7] based on the coset $S O(5) / S O(4)$. It represents the framework where to study, in a computable way, the effects of the lowest lying resonances, both bosonic and fermionic, beyond the leading order chiral Lagrangian approach (this was also done in [15] with a 
different construction). As intimated already, the 4DHCM can be schematised in two sectors, the elementary and the composite one, arising from the extreme deconstruction of the $5 \mathrm{D}$ theory ${ }^{6}$. The reason for this two-site truncation is that it describes only the new states which might be accessible to the LHC while capturing all the relevant features of the composite Higgs models with the Higgs state as a PNGB. The symmetry structure is $S O(5) / S O(4)$ with four Goldstone Bosons (GBs) in the vector representation of $S O(4)$, containing the Higgs boson. The breaking is parametrised by the Vacuum Expectation Value (VEV) of a vector of $S O(5)$.

\subsection{Gauge sector}

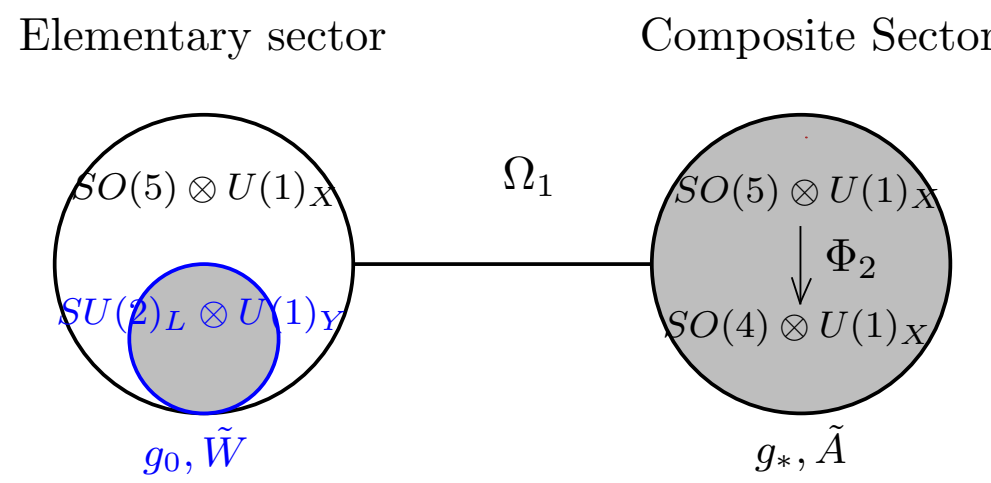

Figure 1: Gauge sector of the 4DCHM. The first site represents the elementary sector, the second the composite sector with $S O(5) \otimes U(1)_{X}$ gauge fields. The grey circles correspond to gauge symmetries.

The elementary sector of the $4 \mathrm{DCHM}$ is associated with the SM gauge symmetry $S U(2)_{L} \otimes U(1)_{Y}$ while the composite sector with the gauge symmetry $S O(5) \otimes U(1)_{X}$ spontaneously broken to $S O(4) \otimes U(1)_{X}$, as shown in Fig. 1. Indicated in a generalised way with $\left(g_{0}, \tilde{W}\right)$ and $\left(g_{*}, \tilde{A}\right)$, respectively, are the couplings and fields of the elementary and composite site7. The gauge Lagrangian for the 4DCHM turns out to be:

$$
\mathcal{L}_{\text {gauge }}=\frac{f_{1}^{2}}{4} \operatorname{Tr}\left|D_{\mu} \Omega_{1}\right|^{2}+\frac{f_{2}^{2}}{2}\left(D_{\mu} \Phi_{2}\right)\left(D_{\mu} \Phi_{2}\right)^{T}-\frac{1}{4} \rho_{\mu \nu}^{A} \rho^{A \mu \nu}-\frac{1}{4} F_{\mu \nu}^{A} F^{A \mu \nu}
$$

where

- the covariant derivatives are defined by:

$$
\begin{aligned}
D^{\mu} \Omega_{1} & =\partial^{\mu} \Omega_{1}-i g_{0} \tilde{W} \Omega_{1}+i g_{*} \Omega_{1} \tilde{A} \\
D_{\mu} \Phi_{2} & =\partial_{\mu} \Phi_{2}-i g_{*} \tilde{A} \Phi_{2}
\end{aligned}
$$

\footnotetext{
${ }^{6}$ This follows the lines of [16] where, however, the full gauge/Goldstone boson structure of the theory is not incorporated.

${ }^{7}$ With $g_{0}$ and $\tilde{W}$ we indicate the couplings and fields of $S U(2)_{L}$ and $U(1)_{Y}$, so that $g_{0}=\left\{g_{0}, g_{0 Y}\right\}$ and $\tilde{W}=\left\{\tilde{W}_{i}, \tilde{Y}\right\}, i=1,2,3$. In the same way with $\tilde{A}$ we indicate all the gauge fields of $S O(5) \otimes U(1)_{X}$.
} 
- the fields $\Omega_{n}, n=1,2$, are link fields responsible for the symmetry breaking and in the unitary gauge they are given by:

$$
\Omega_{n}=\mathbf{1}+i \frac{s_{n}}{h} \Pi+\frac{c_{n}-1}{h^{2}} \Pi^{2}, \quad s_{n}=\sin \left(f h / f_{n}^{2}\right), \quad c_{n}=\cos \left(f h / f_{n}^{2}\right), \quad h=\sqrt{h^{\hat{a}} h^{\hat{a}}}
$$

where $\Pi=\sqrt{2} h^{\hat{a}} T^{\hat{a}}$ is the GB matrix with $T^{\hat{a}}$ the broken generators $(\hat{a}=1,2,3,4)$,

$$
\Pi=\sqrt{2} h^{\hat{a}} T^{\hat{a}}=-i\left(\begin{array}{cc}
0_{4} & \mathbf{h} \\
-\mathbf{h}^{T} & 0
\end{array}\right), \quad \mathbf{h}^{T}=\left(h_{1}, h_{2}, h_{3}, h_{4}\right),
$$

wherein $\mathbf{h}$ is related to the usual Higgs doublet, $H$, via the relation

$$
H=\frac{1}{\sqrt{2}}\left(\begin{array}{c}
-i h_{1}-h_{2} \\
-i h_{3}+h_{4}
\end{array}\right)
$$

where the $f_{i}$ 's are the link coupling constants and the strong sector scale $f$ is given by

$$
\sum_{n=1}^{2} \frac{1}{f_{n}^{2}}=\frac{1}{f^{2}}
$$

- the field $\Phi_{2}$ is a vector of $S O(5)$ that describes the spontaneous symmetry breaking of $S O(5) \otimes U(1)_{X} \rightarrow S O(4) \otimes U(1)_{X}$ and is defined as

$$
\Phi_{2}=\phi_{0} \Omega_{2}^{T} \quad \text { where } \quad \phi_{0}^{i}=\delta^{i 5}
$$

- the last two terms are the kinetic terms of the composite and elementary gauge fields, respectively.

\section{$2.2 \quad$ Fermionic sector}

In the $4 \mathrm{DCHM}$ the SM fermions couple to fermionic operators in the $\mathbf{5}$ of $S O(5)$. This choice of representation is a realistic scenario compatible with precision EW measurements and represents a discretisation to two sites of the model in [17] (see Fig. 2). The new heavy fermions are embedded in the fundamental representation of $S O(5) \otimes U(1)_{X}$ : the spectrum contains four 5 representations indicated with $\Psi_{T, \tilde{T} / B, \tilde{B}}$ in the composite top/bottom sector, respectively. The SM third generation quarks, both for the lefthanded doublet, $q_{L}^{e l}$, and the two right-handed singlets, $b_{R}^{e l}$ and $t_{R}^{e l}$, are embedded in an incomplete representation of $S O(5) \otimes U(1)_{X}$ in such a way that their correct quantum numbers under $S U(2)_{L} \otimes U(1)_{X}$ are reproduced via the relation $Y=T^{3 R}+X$. Here, for illustration purposes, we are considering only the third generation quarks, which are relevant for the computation of the effective potential and for the upcoming phenomenological analysis. The fermionic Lagrangian of the 4DCHM considered in [9] is (for simplicity we take $m_{T}=m_{\tilde{T}}=m_{B}=m_{\tilde{B}}=m_{*}$ ):

$$
\begin{aligned}
\mathcal{L}_{\text {fermions }} & =L_{\text {fermions }}^{\text {el }}+\left(\Delta_{t_{L}} \bar{q}_{L}^{e l} \Omega_{1} \Psi_{T}+\Delta_{t_{R}} \bar{t}_{R}^{e l} \Omega_{1} \Psi_{\tilde{T}}+\text { h.c. }\right) \\
& +\bar{\Psi}_{T}\left(i \hat{D}^{\tilde{A}}-m_{*}\right) \Psi_{T}+\bar{\Psi}_{\tilde{T}}\left(i \hat{D}^{\tilde{A}}-m_{*}\right) \Psi_{\tilde{T}} \\
& -\left(Y_{T} \bar{\Psi}_{T, L} \Phi_{2}^{T} \Phi_{2} \Psi_{\tilde{T}, R}+m_{Y_{T}} \bar{\Psi}_{T, L} \Psi_{\tilde{T}, R}+\text { h.c. }\right) \\
& +(T \rightarrow B)
\end{aligned}
$$


where with $D^{\tilde{A}}$ we indicate the covariant derivative related to the composite gauge fields $\tilde{A}, \Delta_{t_{L}, t_{R}, b_{L}, b_{R}}$ are the mixing parameters relating the elementary and the composite sector whilst $Y_{T, B}$ and $m_{Y_{T, B}}$ are the Yukawas of the composite sector.

For the analytical expressions of the masses of the gauge bosons, their couplings to the quarks and leptons and also the masses of top, bottom and heavy fermionic resonances, we refer to Appendix A.

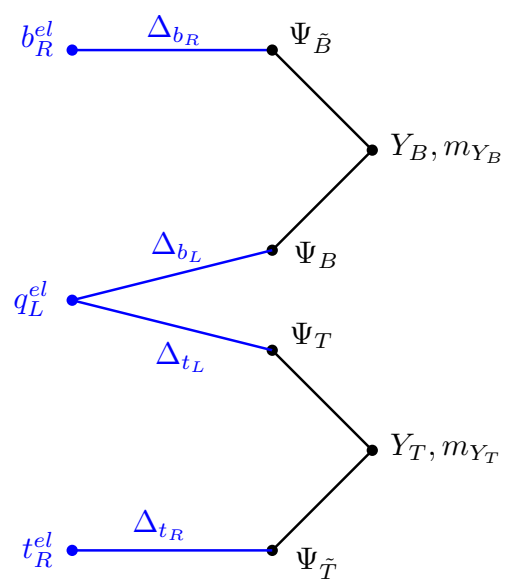

Figure 2: Fermionic sector of the 4DCHM. The elementary sector is on the left, the composite sector with the fermions embedded in fundamental representations of $S O(5) \otimes$ $U(1)_{X}$ is on the right. Symbolically showed are the mixing and Yukawa terms.

\subsection{Higgs sector}

In the 4DCHM the Higgs boson arises as a PNGB through the breaking of $S O(5) \otimes U(1)_{X}$ to $S O(4) \otimes U(1)_{X}$. The gauging of the EW interaction breaks, weakly but explicitly, the strong sector symmetry, as a consequence the Higgs is only approximately a GB and a potential is generated starting at one-loop. With our choice of the fermionic sector, namely, with complete multiplets of the new fermions, the potential turns out to be finite [9]. From the location of the minimum of the potential one extracts the expression for the mass of the Higgs boson, $m_{H}$, and its VEV, $\langle h\rangle$, in terms of the parameters of the model. Also, by defining:

$$
\Omega_{n}=\mathbf{1}+\delta \Omega_{n},
$$

and using eqs. (1) and (9), we can extract the Higgs interactions with the gauge and fermion fields. They are described by the following Lagrangians:

$$
\begin{aligned}
\mathcal{L}_{\text {gauge }, H} & =-\frac{f_{1}^{2}}{2} g_{0} g_{*} T r\left[\tilde{W} \delta \Omega_{1} \tilde{A}+\tilde{W} \tilde{A} \delta \Omega_{1}^{T}+\tilde{W} \delta \Omega_{1} \tilde{A} \delta \Omega_{1}^{T}\right] \\
& +\frac{f_{2}^{2}}{2} g_{*}^{2} \phi_{0}^{T} \delta \Omega_{2}^{T} \tilde{A} \tilde{A} \phi_{0}+\frac{f_{2}^{2}}{2} g_{*}^{2} \phi_{0}^{T} \tilde{A} \tilde{A} \delta \Omega_{2} \phi_{0} \\
& +\frac{f_{2}^{2}}{2} g_{*}^{2} \phi_{0}^{T} \delta \Omega_{2}^{T} \tilde{A} \tilde{A} \delta \Omega_{2} \phi_{0},
\end{aligned}
$$




$$
\begin{aligned}
\mathcal{L}_{\text {ferm }, H} & =\Delta_{t_{L}} \bar{q}_{L}^{e l} \delta \Omega_{1} \Psi_{T}+\Delta_{t_{R}} \bar{t}_{R}^{e l} \delta \Omega_{1} \Psi_{\tilde{T}} \\
& -Y_{T} \bar{\Psi}_{T, L}\left(\phi_{0}^{T} \phi_{0} \delta \Omega_{2}^{T}+\delta \Omega_{2} \phi_{0} \phi_{0}^{T}+\delta \Omega_{2} \phi_{0}^{T} \phi_{0} \delta \Omega_{2}^{T}\right) \Psi_{\tilde{T}, R} \\
& +(T \rightarrow B)+\text { h.c. }
\end{aligned}
$$

The theoretical setup of the 4DCHM allows one to compute a finite Higgs potential with the minimal number of degrees of freedom (in this sense it is the most economical one in the class of composite Higgs model), the latter being dictated by the symmetries of the theory. From the expression of the potential it is possible to extract both the VEV and the mass of the Higgs boson as shown in [9] and [18. For this reason, we will adopt the effective description of the 4DCHM for our phenomenological analysis of DY processes at the LHC.

\section{Model implementation}

The 4DCHM presents a large number of new particles of both kinds, bosons and fermions. In the following ${ }^{8}$ we summarise the particle content.

- Standard Model particles

- SM leptons: $l_{1,2,3}$ and $\nu_{1,2,3}$ (the labels 1, 2, 3 correspond to the flavours $e, \mu$, $\tau$, respectively).

- SM light quarks: $u_{1,2}$ and $d_{1,2}$ (the labels 1,2 correspond to the flavours $u, c$ and $d, s$, respectively).

- SM heavy quarks: $t$ and $b$.

- SM gauge bosons: $\gamma, Z^{0}$ and $W$.

- New particles?

- Neutral gauge bosons: $Z_{i}$ (with $\left.i=1, \ldots 5\right)$.

- Charged gauge bosons: $W_{i}$ (with $\left.i=1, \ldots 3\right)$.

- Charged $+2 / 3$ fermions: $T_{i}$ (with $i=1, \ldots 8$ ).

- Charged $-1 / 3$ fermions: $B_{i}$ (with $i=1, \ldots 8$ ).

- Charged $+5 / 3$ fermions: $\tilde{T}_{i}$ (with $i=1,2$ ).

- Charged $-4 / 3$ fermions: $\tilde{B}_{i}$ (with $i=1,2$ ).

- The Higgs boson: $H$.

We have used the LanHEP package [19] to automatically derive Feynman rules for the 4DCHM in CalcHEP format [20, 21]. Because of the elaborate off-diagonal structure of the mass-matrices of the gauge fields, we have made use of the SLHAplus library [22]. The correspondence between 4DCHM particle names in this paper and their implementation 


\begin{tabular}{|l|l|}
\hline Model & CalcHEP \\
\hline$l_{1}, l_{2}, l_{3}$ & $\mathrm{e} 1, \mathrm{e} 2, \mathrm{e} 3$ \\
$\nu_{1}, \nu_{2}, \nu_{3}$ & $\mathrm{n} 1, \mathrm{n} 2, \mathrm{n} 3$ \\
$u_{1}, u_{2}, d_{1}, d_{2}$ & $\mathrm{u} 1, \mathrm{u} 2, \mathrm{~d} 1, \mathrm{~d} 2$ \\
$t, b$ & $\mathrm{t} 1, \mathrm{~b} 1$ \\
$\gamma, Z^{0}, W^{+}$ & $\mathrm{Z} 1, \mathrm{Z} 2, \mathrm{~W} 1+$ \\
$Z_{1}, \ldots, Z_{5}$ & $\mathrm{Z} 3, \ldots, \mathrm{Z} 7$ \\
$W_{1}^{+}, W_{2}^{+}, W_{3}^{+}$ & $\mathrm{W} 2+, \mathrm{W} 3+, \mathrm{W} 4+$ \\
$T_{1}, \ldots, T_{8}$ & $\mathrm{t} 2, \ldots, \mathrm{t} 9$ \\
$B_{1}, \ldots, B_{8}$ & $\mathrm{~b} 2, \ldots, \mathrm{b} 9$ \\
$\tilde{T}_{1}, \tilde{T}_{2}$ & $\mathrm{n} 1, \mathrm{nn} 2$ \\
$\tilde{B}_{1}, \tilde{B}_{2}$ & $\mathrm{~mm} 1, \mathrm{~mm} 2$ \\
$H$ & $\mathrm{H}$ \\
\hline
\end{tabular}

Table 1: The correspondence between 4DCHM particle names and their implementation in the CalcHEP implementation. In the CalcHEP notation capital letters for fermions corresponds to antiparticles.

in the CalcHEP model is presented in Tab. 1. The parameter space of the 4DCHM, as defined in Sect. 2, is defined in terms of 13 variables. Namely:

$$
f, g_{*}, g_{0}, g_{0 Y}, m_{*}, \Delta_{t_{L}}, \Delta_{t_{R}}, Y_{T}, M_{Y_{T}}, \Delta_{b_{L}}, \Delta_{b_{R}}, Y_{B}, M_{Y_{B}} .
$$

The notation of these parameters in the CalcHEP implementation is given in Tab. 2. The 4DCHM will be publicly available on the High Energy Physics Model Data-Base

\begin{tabular}{|c|l||l|l|}
\hline Model & CalcHEP & Model & CalcHEP \\
\hline$f$ & $\mathrm{f}$ & $m_{*}$ & $\mathrm{~mm}$ \\
$g_{*}$ & $\mathrm{~g}$ & $\Delta_{t_{L}}$ & $\mathrm{DTL}$ \\
$g_{0}$ & $\mathrm{~g} 0$ & $\Delta_{t_{R}}$ & $\mathrm{DTR}$ \\
$g_{0 Y}$ & $\mathrm{~g} 0 \mathrm{y}$ & $Y_{T}$ & $\mathrm{YT}$ \\
$\langle h\rangle$ & $\mathrm{h}$ & $M_{Y_{T}}$ & $\mathrm{MYT}$ \\
& & $\Delta_{b_{L}}$ & $\mathrm{DBL}$ \\
& & $\Delta_{b_{R}}$ & $\mathrm{DBR}$ \\
& & $Y_{B}$ & $\mathrm{YB}$ \\
& & $M_{Y_{B}}$ & $\mathrm{MYB}$ \\
& & $m_{H}$ & $\mathrm{MH}$ \\
\hline
\end{tabular}

Table 2: The notation of the 4DCHM parameters in the CalcHEP implementation.

(HEPMDB) [23] at https://hepmdb.soton.ac.uk/ by November 2012 under the "4DCHM" name.

\footnotetext{
${ }^{8}$ As clear from our previous Section, hereafter we will distinguish between SM first and second quark families and the third one, the latter being formed by quasi-composite particles.

${ }^{9} \mathrm{An}$ increasing number labelling the $4 \mathrm{DCHM}$ bosonic and fermionic states corresponds to their increasing masses.
} 
We will use as input the following physical quantities: $e, M_{Z}, G_{F}, m_{t}, m_{b}, m_{H}$. In order to analyse the parameter space of the 4DCHM in presence of such constraints, we have written a standalone Mathematica program [24] that is able to sort allowed points by varying the model parameters in a fixed range. Specifically, we have considered $f$ (the strong coupling scale) and $g_{*}$ (the gauge coupling constant of the extra gauge group) as free parameter while we have scanned over $m_{*}, \Delta_{t_{L}}, \Delta_{t_{R}}, Y_{T}, M_{Y_{T}}, \Delta_{b_{L}}, \Delta_{b_{R}}, Y_{B}$ and $M_{Y_{B}}$.

We have accounted for the above constraints in the following way: from the gauge sector, by diagonalising the charged and neutral mass matrix, we have constrained the value of $g_{0}, g_{0 Y}$ and $\langle h\rangle$ with respect to the electric charge, the mass of the $Z$ boson and the value of the Fermi constant leaving, as intimated, $f$ and $g_{*}$ as free parameters. In the fermionic sector, due to the large number of parameters, we have performed a random scan, with the value of $f$ and $g_{*}$ chosen from the gauge sector, over $m_{*}, \Delta_{t_{L}}, \Delta_{t_{R}}, Y_{T}, M_{Y_{T}}, \Delta_{b_{L}}, \Delta_{b_{R}}, Y_{B}$ and $M_{Y_{B}}$, imposing to reconstruct the mass of the top and bottom quarks and of the Higgs boson. Namely, by taking into account the $t$-quark data from LEP, SLC, Tevatron and LHC we have required $165 \mathrm{GeV} \leq m_{t} \leq 175$ $\mathrm{GeV}$ on the top quark running mass, $2 \mathrm{GeV} \leq m_{b} \leq 6 \mathrm{GeV}$ on the bottom quark running mass and we have assumed a conservative interval $124 \mathrm{GeV} \leq m_{H} \leq 126 \mathrm{GeV}$ for the Higgs mass, which is compliant with the observation of a SM-like Higgs boson as recently claimed by the ATLAS [11] and CMS [12] experiments ${ }^{10}$.

The gauge resonance masses, couplings and widths for the ensuing DY analysis have been computed using sets of parameters generated in the same way. The calculation of the masses, couplings and widths has of course been double-checked between CalcHEP and the Mathematica program.

As for event generation, the codes exploited for our study of the LHC signatures is based on helicity amplitudes, defined through the HELAS subroutines [26] and assembled by means of MadGraph [27], which has been validated against CalcHEP. The Matrix Elements (MEs) generated account for all off-shellness effects of the particles involved. Two different phase space implemetations were used, an 'ad-hoc one' (based on Metropolis [28]) and a 'blind one' based on RAMBO [29], checked one against the other. The latter was adopted eventually, as it proved the most unbiased one in sampling the multiple resonances existing in each (neutral and charged current) DY channel. Further, VEGAS [30] was finally used for the multi-dimensional numerical integrations. All these additional subroutines were also validated against CalcHEP outputs.

The MEs have been computed at Leading Order (LO). Clearly, in the LHC environment, QCD corrections are not negligible and associated scale uncertainties may impact on the dynamics of $Z^{\prime}$ and $W^{\prime}$ production and decay (see Ref. [31] for the case of the SM $Z$ and $W$ channels). In fact, EW corrections may also be relevant [32, 33. However, the treatment we are adopting here of the two DY channels is such that real radiation of gluons/photons would be treated inclusively (i.e., no selection is enforced here that relies on the gluon/photon dynamics), so that we do not expect such QCD effects to impact on the distributions that we will be considering, neither those of the cross sections nor those of the asymmetries, apart from an overall rescaling. The latter, in particular, when implemented at large invariant/transverse mass, is affected by a residual uncertainty of $5 \%$ at the most [32, 33].

\footnotetext{
${ }^{10}$ Tevatron $[25$ has also reported results that are consistent with the LHC observations in this respect.
} 
The Parton Distribution Functions (PDFs) used were CTEQ5L [34], with factorisation/renormalisation scale set to $Q=\mu=\sqrt{\hat{s}}$. (We have verified that later PDF sets do not generate any significant difference in the results we are going to present ${ }^{11}$.) Initial state quarks have been taken as massless, just like the final state leptons and neutrinos.

Furthermore we have checked that our 4DCHM parameter choices, for any sorted point, are compatible with LHC direct searches for heavy gauge bosons [36, 37, 38, 39] and fermions [40, 41, 42, 43].

Let us now comment about the bounds from the EW Precision Tests (EWPTs) on the 4DCHM [44]. As it is well known, extra gauge bosons give a positive contribution to the Peskin-Takeuchi $S$ parameter and the requirement of consistency with the EWPTs generally gives a bound on the mass of these resonances around few $\mathrm{TeV}$. In contrast, the fermionic sector is quite irrelevant for $S$ since the extra fermions are weakly coupled to the SM gauge bosons. Either way, as noticed in [17], when dealing with effective theories, one can only parametrise $S$ rather than calculating it. In other words, since we are dealing with a truncated theory describing only the lowest-lying resonances that may exist, we need to invoke an Ultra-Violet (UV) completion for the physics effects we are not including in our description. These effects could well compensate for $S$, albeit with some tuning. One example is given in [17] by considering the contribution of higher-order operators in the chiral expantion. Another scenario leading to a reduced $S$ parameter is illustrated in [9], by including non-minimal interactions in the 4DCHM.

Nevertheless, in the following phenomenological analysis, we will chose values for the gauge resonance masses around $2 \mathrm{TeV}$ or larger in order to avoid too big contributions to the $S$ parameter. Recalling that, in the $4 \mathrm{DCHM}$, the mass scale of the lightest gauge boson resonance is given by

$$
M_{\text {lightest }}=f g_{*},
$$

we feel justified in choosing for $f$, the compositeness scale of the model, values around 1 $\mathrm{TeV}$ and for $g_{*}$, the coupling constant of the composite gauge sector, values around 2 .

Concerning the fermionic sector, our setup is constructed in such a way as to avoid leading-order corrections to the $Z b \bar{b}$ coupling. As a consequence, ones does not experience substantial bounds on the fermion parameters, so that we will vary them in order to easily reconstruct the top and bottom quark masses (as previously detailed).

\section{Parameter space and benchmarks}

\section{1 $\quad Z^{\prime}$ and $W^{\prime}$ decays}

Other than the masses of the new gauge bosons, since the latter appear in DY processes at the LHC as resonances, we are interested here also in their decay widths. Due to the high number of parameters in the fermionic sector, we can have different regimes in analysing the widths of the new gauge resonances. We can have a configuration, (i), in which the mass of the fermionic resonances is too heavy to have a decay of a gauge resonance in pairs of heavy fermions (so that, other than the SM-like decay channels

\footnotetext{
${ }^{11}$ Furthermore, we have estimated the theoretical uncertainty (at NLO) due to the PDFs by adopting NNPDF sets [35, which yielded a $10 \%$ effect at the most, rather independent of the $Z^{\prime}$ and $W^{\prime}$ masses involved and with negligible impact onto the shape of the differential distributions presented.
} 
also the mixed ones, involving an elementary and a composite fermion, are open) or the opposite configuration, (ii), in which the mass of the fermionic resonances (typically that of the lightest one) is small enough so that this is possible. In the former case the typical decay widths can be well below $100 \mathrm{GeV}$ whereas in the latter case they can grow significantly, to become even comparable to the masses themselves. So, typically, we can analyse the Branching Ratios (BRs) of the gauge resonances in three well-defined and distinct situations.

1. The threshold for the gauge boson decays in pairs of heavy fermions has not been reached, therefore case (i) above is realised: hereafter, 'small width' regime.

2. The threshold for the gauge boson decays in pairs of heavy fermions has just been reached, therefore case (ii) above is realised, where however phase space effects are not yet predominant: hereafter, 'medium width' regime.

3. The threshold for the gauge boson decays in pairs of heavy fermions has been abundantly surpassed, therefore case (ii) above is realised, where indeed phase space effects are becoming important: hereafter, 'large width' regime.

Despite such configurations emerge for whichever combination of the aforementioned $f$ and $g_{*}$ parameters of the new gauge sector that we studied and we will use two setups to illustrate this $\left(f=0.8 \mathrm{TeV}\right.$ and $g_{*}=2.5$ versus $f=1.2 \mathrm{TeV}$ and $\left.g_{*}=1.8\right)$, we will study the emerging decay dynamics in detail only for one benchmark combination of these two parameters $\left(f=1.2 \mathrm{TeV}\right.$ and $\left.g_{*}=1.8\right)$. However, we will eventually present results not only for both these benchmarks but also for various other pairings of $f$ and $g_{*}$ when we will study possible LHC observables.

It is in order at this point to attempt quantifying the naturalness of the various $f$ and $g_{*}$ combinations studied, within the 4DCHM. In order to do so, we have run 4DCHM parameter scans with $f$ and $g_{*}$ fixed to the following combinations: (a) $f=0.75 \mathrm{TeV}$ and $g^{*}=2$; (b) $f=0.8 \mathrm{TeV}$ and $g^{*}=2.5$; (c) $f=1 \mathrm{TeV}$ and $g^{*}=2$; (d) $f=1$ $\mathrm{TeV}$ and $g^{*}=2.5$; (e) $f=1.1 \mathrm{TeV}$ and $g^{*}=1.8$; (f) $f=1.2 \mathrm{TeV}$ and $g^{*}=1.8$. All other parameters (with reference to their definitions in section 2) were varied over the following intervals: $m_{*}, \Delta_{t L}, \Delta_{t R}, Y_{T}, M_{Y_{T}}, Y_{B}$ and $M_{Y_{B}}$ between 0.5 and $5 \mathrm{TeV}$ while $\Delta_{b L}$ and $\Delta_{b R}$ between 0.05 and $0.5 \mathrm{TeV}$ (in the spirit of 'partial compositness' [9]). All aforementioned theoretical and experimental constraints were implemented in each case (though we notice that those on the $b$ - and $t$-quark masses and couplings have minimal impact). The total number of random points generated for each $f$ and $g_{*}$ combination was of order 15 millions. As the scans were not optimised, i.e., all points were equi-probable, a simple yet good measure of naturalness is just the percentage of points surviving in each scans. The typical survival rate is of $\mathcal{O}\left(2 \div 3 \times 10^{-7}\right)$, with variations amongst the various $f$ and $g_{*}$ combinations of no more than $30 \%$. We therefore notice, on the one hand, that the 4DCHM is highly constrained already by current theoretical and experimental bounds (hence amenable to stringent phenomenological investigation) and, on the other hand, that none of our choices of $f$ and $g_{*}$ is overwhelmningly more unnatural than others (in fact, we also reassuringly reconfirm that with increasing $f$ we have a decreasing number of points found [9]). 


\subsubsection{Spectrum with $f=1.2 \mathrm{TeV}$ and $g_{*}=1.8$}

With these values of the gauge parameters, the lightest gauge resonances are slightly heavier than $2 \mathrm{TeV}$ while the heaviest ones are very close to $3 \mathrm{TeV}$, for both the neutral and the charged sector, so as to not run afoul of recent Tevatron and LHC constraints in searching for generic $W^{\prime}$ [36, 37] and $Z^{\prime}$ [38, 39] states.

As already noticed, the masses of the neutral and charged gauge resonances depend only on $f$ and $g_{*}$ so these are the same in the various width regimes. Recalling that two of the $Z^{\prime}$ states and one of the $W^{\prime}$ states are coupled neither to light quarks nor to leptons, i.e., $Z_{1}$ and $Z_{4}$ for the neutral sector plus $W_{1}$ for the charged sector ${ }^{12}$, we are only interested here in the cases $Z_{i}$ with $i=2,3,5$ and $W_{i}$ with $i=2,3$, whose masses are reported in Tab. 3. In Figs. 3 and 4 we show instead the widths of the gauge bosons $Z_{2}, Z_{3}, Z_{5}$ and $W_{2}, W_{3}$, respectively, as a function of the lightest fermionic mass resonance of charge $2 / 3$, that is $T_{1}$. By definition this is the lightest non-exotic heavy fermion of the $4 \mathrm{DCHM}$ of charge $+2 / 3$, essentially degenerate in mass with its charge $-1 / 3$ counterpart (i.e., $B_{1}$ ) and they are generally lighter than their exotic 'cousins': that is, $\tilde{T}_{1}$ (charge $\left.+5 / 3\right)$ and $\tilde{B}_{1}$ (charge $\left.-4 / 3\right)$. The two different coloured regions in the plots correspond to the aforementioned situations (i) and (ii): in green squares we have the points where the decay of the heavy gauge bosons in two heavy fermions $F \bar{F}$ is forbidden while in black circles we have the points where these decays are permitted. What is remarkable to notice here (and this comment can be extended to the other $f$ and $g_{*}$ combinations studied) is that these two cases correspond to two almost distinct populations in the plots, separated roughly at the point $M_{\text {lightest }} \approx 2 m_{T_{1}} \approx 2 m_{B_{1}}$, with a minimum of 'leakage' of one into the other. Therefore, from a phenomenological point of view, to be able to measure a $Z^{\prime}$ and/or $W^{\prime}$ width below, say, $100 \mathrm{GeV}$, would signify the existence of some heavy fermions with a mass scale not below $\mathcal{O}\left(f g_{*} / 2\right)$ and up to $\mathcal{O}\left(f g_{*}\right)$ (or indeed above) (case (i), regime 1.). Conversely, to measure widths in excess of this value would point towards the existence of such additional fermionic states at rather low mass. In fact, one may further argue, albeit limited to the case of $Z_{2}$ or $Z_{3}$ (see top two plots in Fig. 3) and $W_{2}$ (see left plot in Fig. 4), that two sub-populations exist for case (ii), one corresponding to widths below $600 \mathrm{GeV}$ or so, and one above it (regimes 2 . and 3. above, respectively).

\begin{tabular}{|l|l||l|l|}
\hline & Mass $(\mathrm{GeV})$ & & Mass $(\mathrm{GeV})$ \\
\hline$Z_{2}$ & 2249 & $W_{2}$ & 2312 \\
$Z_{3}$ & 2312 & $W_{3}$ & 3056 \\
$Z_{5}$ & 3056 & & \\
\hline
\end{tabular}

Table 3: Masses of the additional gauge boson resonances in the 4DCHM for the parameter point $f=1.2 \mathrm{TeV}$ and $g_{*}=1.8$.

While such very distinctive spectrum configurations of the 4DCHM open up interesting phenomenological possibilities, it remains to be seen whether these are accessible at the LHC via DY processes. We will address this point later on. In fact, in order to do so, we ought first to define some benchmarks in parameter space, representative of the

\footnotetext{
${ }^{12}$ So that they are essentially 'inert' for the purpose of studying DY processes.
} 

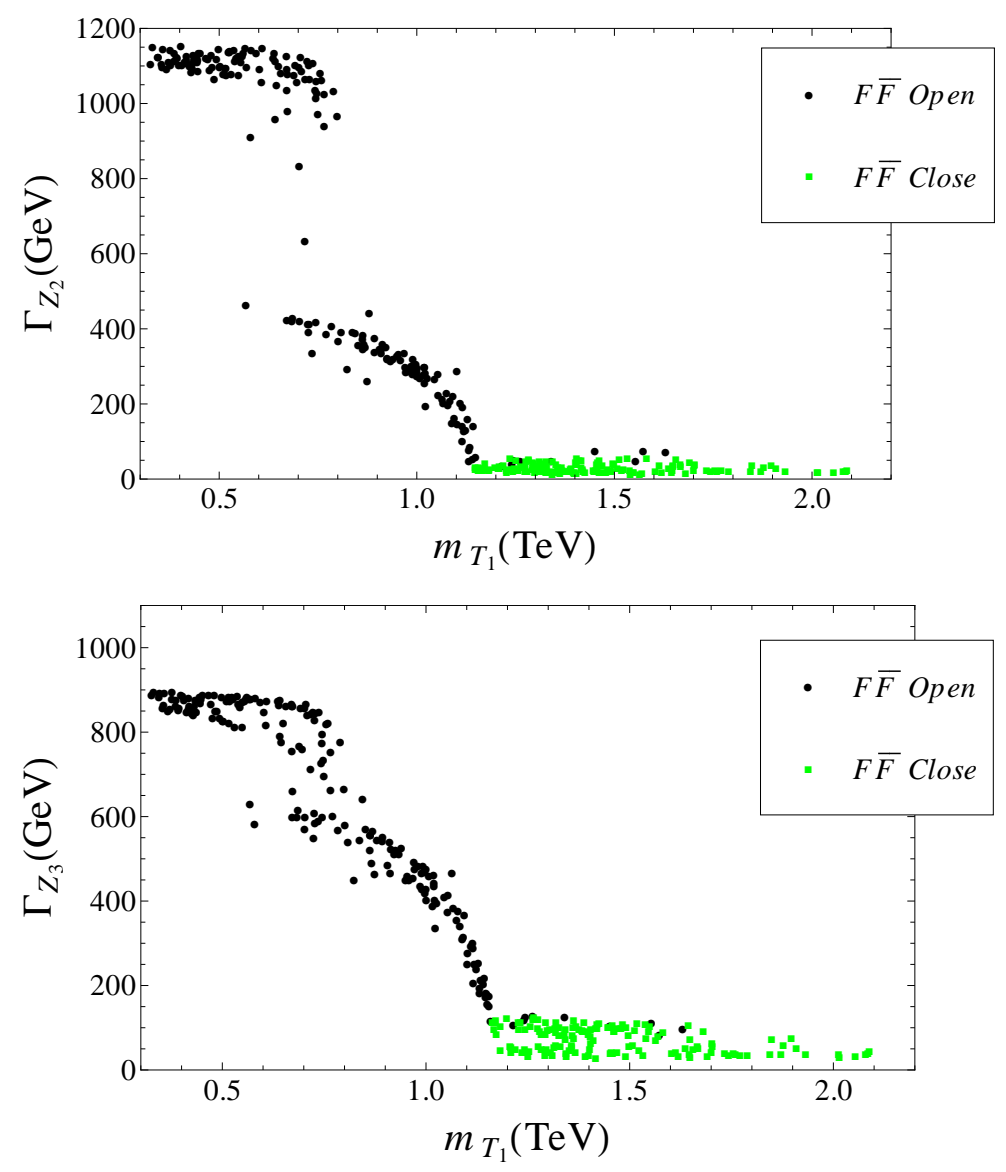

(a)

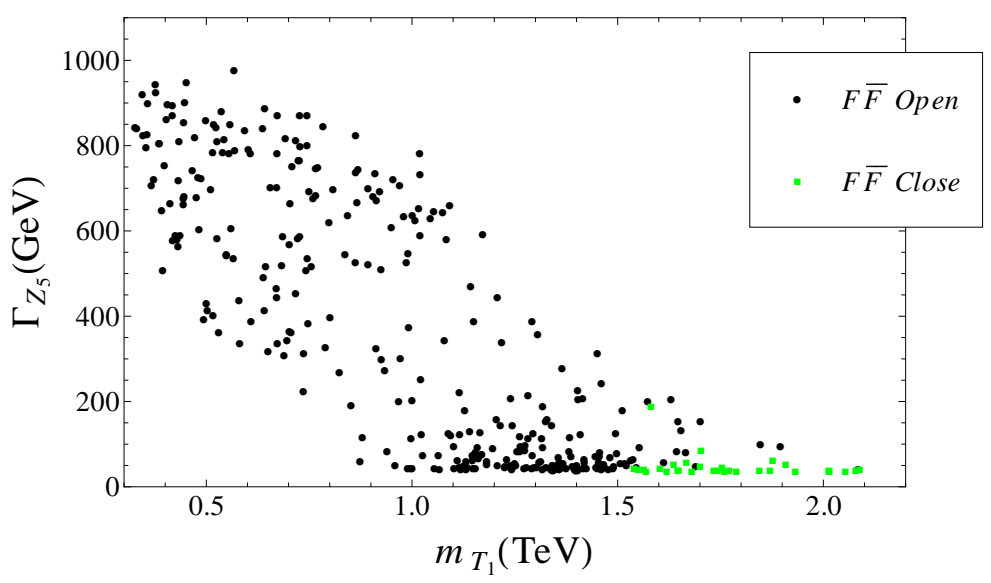

(b)

(c)

Figure 3: Width of the additional neutral gauge bosons of the 4DCHM, for the choice $f=1.2 \mathrm{TeV}$ and $g_{*}=1.8$, (a) for $Z_{2}$ (b) for $Z_{3}$ and (c) for $Z_{5}$, as a function of the mass of the lightest fermionic resonance of charge $+2 / 3$. The circle points in black are the ones where the decay in a pair of heavy fermions is permitted while the square points in green are the ones where this process is forbidden. The fermionic parameters are varied between 0.5 and $5 \mathrm{TeV}$, except $\Delta_{b L}$ and $\Delta_{b R}$ that are varied between 0.05 and $0.5 \mathrm{TeV}$.

three width regimes already described. We shall do so next. Further, we will at the same time list the main decay channels of the gauge boson resonances in these various regimes of their widths. 

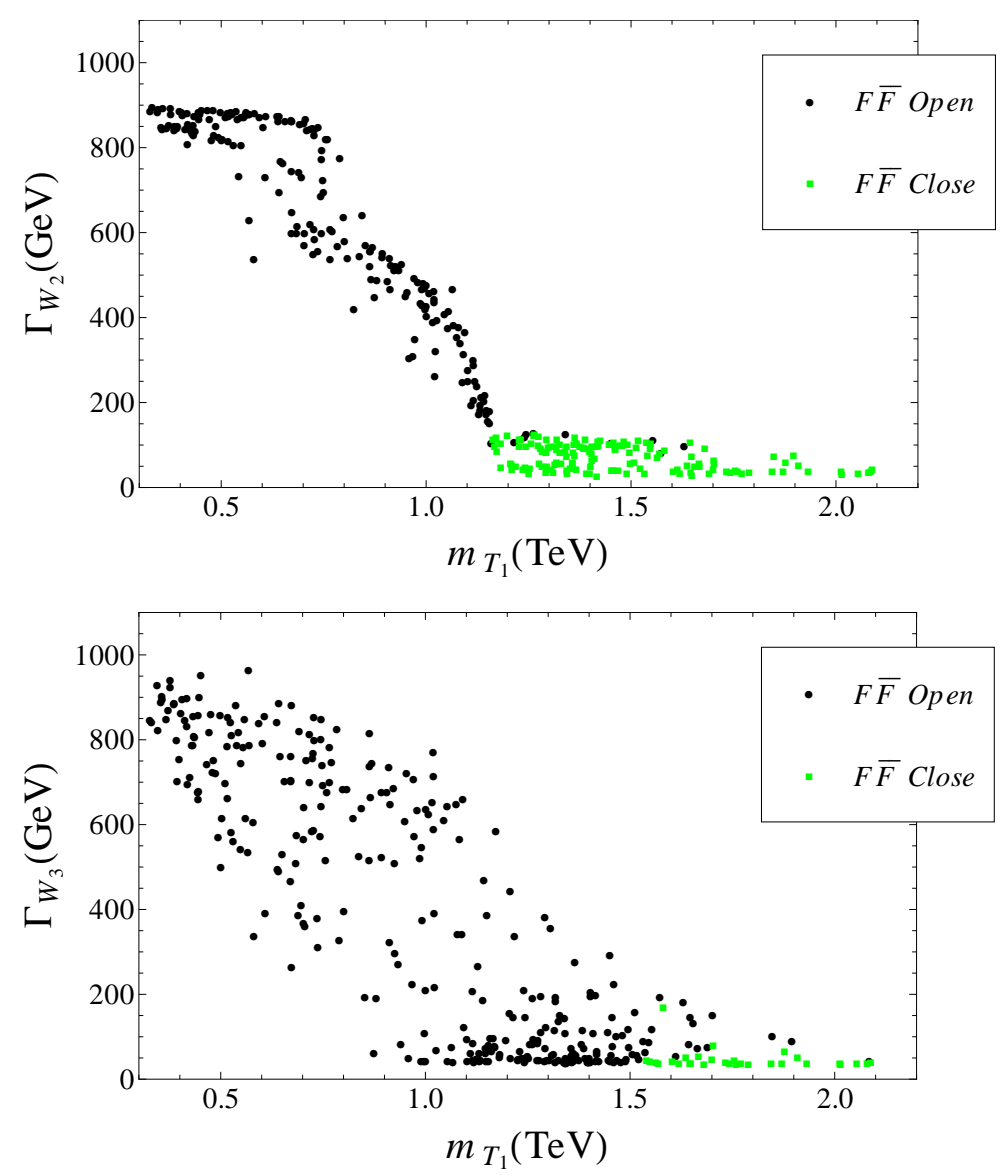

(a)

(b)

Figure 4: Width of the additional charged gauge bosons of the 4DCHM, for the choice $f=1.2 \mathrm{TeV}$ and $g_{*}=1.8$, (a) for $W_{2}$ and (b) for $W_{3}$, as a function of the mass of the lightest fermionic resonance of charge $+2 / 3$. The circle points in black are the ones where the decay in a pair of heavy fermions is permitted while the square points in green are the ones where this process is forbidden. The fermionic parameters are varied between 0.5 and $5 \mathrm{TeV}$, except $\Delta_{b L}$ and $\Delta_{b R}$ that are varied between 0.05 and $0.5 \mathrm{TeV}$.

\subsection{1.a Small width regime}

In the small width regime the masses of the heavy fermions, the width for the (accessible) $Z^{\prime}$ and $W^{\prime}$ states and their BRs are reported in Tabs. 5, 6, 7 and 8 and are generated with the benchmark in Tab. 4. The characteristics of the benchmark point illustrated therein are representative of a typical pattern emerging from this populations of points.

In particular, in this regime we see that the main decay channels of the lightest $Z^{\prime} \mathrm{s}$ (i.e., $Z_{2}$ and $Z_{3}$ ) and $W^{\prime}$ (i.e., $W_{2}$ ) are in SM fermions and gauge bosons, yet we can also have decay channels in one SM fermion and one heavy fermionic resonance, albeit at the percent level at the most. In the case of the heavy $Z^{\prime}$ (i.e., $Z_{5}$ ) and $W^{\prime}$ (i.e., $W_{3}$ ) the corresponding rates grow significantly, up to the ten percent level or more. Interestingly, both $Z_{5}$ and $W_{3}$ can also decay in lighter gauge (and Higgs) boson states and, whenever they do, the corresponding rates are quite dominant. Further, $Z_{2}$ and $Z_{3}$ decays into $Z^{0} H$ plus $W_{2}$ decays into $W H$ are also active, at the $10 \%$ level, possibly leading to new 


\begin{tabular}{|l||l||l|}
\hline$Z_{2}$ & $Z_{3}$ & $Z_{5}$ \\
\hline $68 \%$ in $t \bar{t}$ & $33 \%$ in $b \bar{b}$ & $11 \%$ in $W^{+} W_{1,2}^{-}$and c.c. \\
$9 \%$ in $W^{+} W^{-}$and $10 \%$ in $Z^{0} H$ & $30 \%$ in $t \bar{t}$ & $11 \%$ in $Z_{3} H$ \\
$5 \%$ in $b \bar{b}$ & $11 \%$ in $W^{+} W^{-}$and $10 \%$ in $Z^{0} H$ & $6.5 \%$ in $t \bar{T}_{4}$ and c.c. \\
$1 \%$ in $u_{1,2} \bar{u}_{1,2}$ & $2.5 \%$ in $d_{1,2} \bar{d}_{1,2}$ and $u_{1,2} \bar{u}_{1,2}$ & $6 \%$ in $Z_{2} H$ and $Z_{1} H$ \\
$1 \%$ in $l_{1, \ldots 3} \bar{l}_{1, \ldots 3}$ & $1 \%$ in $l_{1, \ldots 3} \bar{l}_{1, \ldots 3}$ & $5 \%$ in $t \bar{T}_{3}$ and c.c. \\
$0.5 \%$ in $d_{1,2} \bar{d}_{1,2}$ & $0.3 \%$ in $t \bar{T}_{2,3}$ and c.c. & $3 \%$ in $t \bar{T}_{5}$ and c.c. \\
$0.2 \%$ in $b \bar{B}_{3}$ and c.c. & & $1.5 \%$ in $t \bar{t}$ \\
$0.1 \%$ in $t \bar{T}_{2}$ and c.c. & & \\
\hline
\end{tabular}

Table 7: BRs of the new neutral gauge bosons of the 4DCHM in the small width regime.

\begin{tabular}{|l||l|}
\hline$W_{2}^{+}$ & $W_{3}^{+}$ \\
\hline $63 \%$ in $t b$ & $13 \%$ in $t \bar{B}_{4}$ \\
$10 \%$ in $W^{+} Z^{0}$ and $10 \%$ in $W^{+} H$ & $12 \%$ in $W_{1}^{+} H$ \\
$5 \%$ in $u_{1,2} \bar{d}_{1,2}$ & $11 \%$ in $W^{+} Z_{3}$ \\
$1.5 \%$ in $\nu_{1, \ldots .} \bar{l}_{1, \ldots 3}$ & $11 \%$ in $W_{1,2}^{+} Z^{0}$ \\
$0.6 \%$ in $T_{3} \bar{b}$ & $11 \%$ in $W_{2}^{+} H$ \\
$0.4 \%$ in $\tilde{T}_{1} \bar{t}$ & $6.5 \%$ in $T_{3} \bar{b}$ \\
& $6 \%$ in $W^{+} Z_{1,2}$ \\
& $5.5 \%$ in $\tilde{T}_{2} \bar{t}$ \\
& $4 \%$ in $T_{4} \bar{b}$ \\
\hline
\end{tabular}

Table 8: BRs of the new charged gauge bosons of the 4DCHM in the small width regime.

\begin{tabular}{|c|l|l|l|}
\hline $\mathrm{f}(\mathrm{GeV})$ & 1200 & $m_{*}(\mathrm{GeV})$ & 2216 \\
$g_{*}$ & 1.8 & $\Delta_{t_{L}}(\mathrm{GeV})$ & 2434 \\
$g_{0}$ & 0.70 & $\Delta_{t_{R}}(\mathrm{GeV})$ & 2362 \\
$g_{0 y}$ & 0.37 & $Y_{T}(\mathrm{GeV})$ & 2771 \\
$\langle h\rangle(\mathrm{GeV})$ & 248 & $M_{Y_{T}}(\mathrm{GeV})$ & -1031 \\
& & $\Delta_{b_{L}}(\mathrm{GeV})$ & 327 \\
& $\Delta_{b_{R}}(\mathrm{GeV})$ & 299 \\
& $Y_{B}(\mathrm{GeV})$ & 2815 \\
& $M_{Y_{B}}(\mathrm{GeV})$ & -4093 \\
& $m_{H}(\mathrm{GeV})$ & 124 \\
\hline
\end{tabular}

Table 9: Medium width benchmark

channels of the heavy gauge bosons, both neutral and charged. However, as clear from comparing the heavy gauge boson and heavy fermion masses relatively, these decays occur just above threshold, so that the $Z^{\prime}$ and $W^{\prime}$ widths, although increasing significantly, remain well below the corresponding mass values, so that these objects would still potentially appear as clear resonances over the SM background. 


\begin{tabular}{|ll||ll|||ll|}
\hline \multicolumn{2}{|c||}{ Mass $(\mathrm{GeV})$} & & Mass $(\mathrm{GeV})$ & & Mass $(\mathrm{GeV})$ \\
\hline$T_{1}$ & 986 & $B_{1}$ & 971 & $\tilde{T}_{1,2}$ & 1759,2790 \\
$T_{2}$ & 1753 & $B_{2}$ & 985 & $\tilde{B}_{1,2}$ & 970,5062 \\
$T_{3}$ & 2113 & $B_{3}$ & 1683 & & \\
$T_{4}$ & 2408 & $B_{4}$ & 2255 & & \\
$T_{5}$ & 2800 & $B_{5}$ & 2950 & & \\
$T_{6}$ & 3408 & $B_{6}$ & 3432 & & \\
$T_{7}$ & 3653 & $B_{7}$ & 5062 & & \\
$T_{8}$ & 5065 & $B_{8}$ & 5065 & & \\
\hline
\end{tabular}

Table 10: Masses of the new fermionic resonances of the 4DCHM in the medium width regime.

\begin{tabular}{|l|l||l|l|}
\hline & Width $(\mathrm{GeV})$ & & Width $(\mathrm{GeV})$ \\
\hline$Z_{2}$ & 301 & $W_{2}$ & 434 \\
$Z_{3}$ & 434 & $W_{3}$ & 522 \\
$Z_{5}$ & 526 & & \\
\hline
\end{tabular}

Table 11: Widths of the new gauge resonances of the 4DCHM in the medium width regime.

\begin{tabular}{|l||l||l|}
\hline$Z_{2}$ & $Z_{3}$ & $Z_{5}$ \\
\hline $46 \%$ in $B_{1} \bar{B}_{1}$ & $24 \%$ in $\tilde{B}_{1} \tilde{B}_{1}$ & $24 \%$ in $B_{1} \bar{B}_{3}$ and c.c. \\
$40 \%$ in $\tilde{B}_{1} \tilde{B}_{1}$ & $22 \%$ in $T_{1} \bar{T}_{1}$ & $20 \%$ in $B_{2} \bar{B}_{3}$ and c.c \\
$8 \%$ in $t \bar{t}$ & $20 \%$ in $B_{2,3} \bar{B}_{2,3}$ & $1 \%$ in $W^{+} W_{1,2}^{-}$and c.c. \\
$2 \%$ in $B_{2} \bar{B}_{2}$ & $4 \%$ in $t \bar{t}$ and $b \bar{b}$ & $1 \%$ in $t \bar{T}_{4}$ and c.c. \\
$1 \%$ in $T_{1} \bar{T}_{1}$ & $1 \%$ in $W^{+} W^{-}$and $Z^{0} H$ & $1 \%$ in $Z_{3} H$ \\
$1 \%$ in $W^{+} W^{-}$and $Z^{0} H$ & $0.5 \%$ in $u_{1,2} \bar{u}_{1,2}$ and $d_{1,2} \bar{d}_{1,2}$ & $0.5 \%$ in $Z_{1,2} H$ \\
$0.6 \%$ in $b \bar{b}$ and c.c. & \\
$0.1 \%$ in $u_{1,2} \bar{u}_{1,2}$ and $l_{1, \ldots 3} \bar{l}_{1, \ldots 3}$ & & \\
\hline
\end{tabular}

Table 12: BRs of the new neutral gauge bosons of the 4DCHM in the medium width regime.

\subsection{1.c Large width regime}

In the large width regime the masses of the heavy fermions, the width for the (accessible) $Z^{\prime}$ and $W^{\prime}$ states and their BRs are reported in Tabs. 15, 16, 17 and 18 and are generated with the benchmark in Tab. 14. The dynamics of the sub-population of which this point is representative is typical, as seen here.

In this regime we see that once again the main decay channels of the $Z^{\prime}$ and $W^{\prime}$ are in heavy fermions (including exotic ones) and that we have the appearance of decays in pairs of heavy fermions different from the lightest ones, unlike the previous case. Regarding this decay phenomenology in general, it is clear that both the new channels opening up 


\begin{tabular}{|l||l|}
\hline$W_{2}^{+}$ & $W_{3}^{+}$ \\
\hline $44 \%$ in $B_{1} \tilde{B}_{1}$ & $45 \%$ in $B_{3} \tilde{B}_{1}$ \\
$42 \%$ in $T_{1} \bar{B}_{2}$ & $43 \%$ in $T_{1} \bar{B}_{3}$ \\
$9 \%$ in $t \bar{b}$ & $1.6 \%$ in $t \bar{B}_{4}$ \\
$1 \%$ in $W^{+} H$ and $W^{+} Z^{0}$ & $1 \%$ in $W_{1,2}^{+} H, W_{1,2} Z^{0}$ and $W^{+} Z_{3}$ \\
$0.5 \%$ in $u_{1,2} \bar{d}_{1,2}$ & $1 \%$ in $t \bar{B}_{3}$ \\
\hline
\end{tabular}

Table 13: BRs of the new charged gauge bosons of the 4DCHM in the medium width regime.

and the phase space effects fully onsetting in the others contribute to render the $Z^{\prime}$ and $W^{\prime}$ widths here very substantial, to the extent that it remains to be seen whether they would actually appear as resonant objects in the detectors.

\begin{tabular}{|c|l|l|l|}
\hline $\mathrm{f}(\mathrm{GeV})$ & 1200 & $m_{*}(\mathrm{GeV})$ & 1293 \\
$g_{*}$ & 1.8 & $\Delta_{t_{L}}(\mathrm{GeV})$ & 4714 \\
$g_{0}$ & 0.70 & $\Delta_{t_{R}}(\mathrm{GeV})$ & 3402 \\
$g_{0 y}$ & 0.37 & $Y_{T}(\mathrm{GeV})$ & 4165 \\
$\langle h\rangle(\mathrm{GeV})$ & 248 & $M_{Y_{T}}(\mathrm{GeV})$ & -1503 \\
& & $\Delta_{b_{L}}(\mathrm{GeV})$ & 224 \\
& $\Delta_{b_{R}}(\mathrm{GeV})$ & 480 \\
& $Y_{B}(\mathrm{GeV})$ & 4260 \\
& & $M_{Y_{B}}(\mathrm{GeV})$ & -2835 \\
& $m_{H}(\mathrm{GeV})$ & 125 \\
\hline
\end{tabular}

Table 14: Large width benchmark

\begin{tabular}{|ll||ll|||ll|}
\hline \multicolumn{2}{|c||}{ Mass $(\mathrm{GeV})$} & & Mass $(\mathrm{GeV})$ & & Mass $(\mathrm{GeV})$ \\
\hline$T_{1}$ & 502 & $B_{1}$ & 502 & $\tilde{T}_{1,2}$ & 744,2247 \\
$T_{2}$ & 740 & $B_{2}$ & 507 & $\tilde{B}_{1,2}$ & 501,3336 \\
$T_{3}$ & 1910 & $B_{3}$ & 865 & & \\
$T_{4}$ & 2232 & $B_{4}$ & 1936 & & \\
$T_{5}$ & 2663 & $B_{5}$ & 2202 & & \\
$T_{6}$ & 3336 & $B_{6}$ & 3336 & & \\
$T_{7}$ & 3878 & $B_{7}$ & 3336 & & \\
$T_{8}$ & 4914 & $B_{8}$ & 4913 & & \\
\hline
\end{tabular}

Table 15: Masses of the new fermionic resonances of the 4DCHM in the large width regime. 


\begin{tabular}{|l||ll||l|}
\hline & Width $(\mathrm{GeV})$ & & Width $(\mathrm{GeV})$ \\
\hline$Z_{2}$ & 1099 & $W_{2}$ & 820 \\
$Z_{3}$ & 827 & $W_{3}$ & 614 \\
$Z_{5}$ & 413 & & \\
\hline
\end{tabular}

Table 16: Widths of the new gauge resonances of the 4DCHM in the large width regime.

\begin{tabular}{|l||l||l|}
\hline$Z_{2}$ & $Z_{3}$ & $Z_{5}$ \\
\hline $31 \%$ in $T_{2} \bar{T}_{2}$ & $17 \%$ in $\tilde{B}_{1} \tilde{B}_{1}$ & $24 \%$ in $B_{1} \bar{B}_{3}$ and c.c. \\
$29 \%$ in $\tilde{B}_{1} \tilde{\tilde{B}}_{1}$ & $16 \%$ in $T_{1} \bar{T}_{1}$ & $6 \%$ in $t \bar{T}_{2}$ and c.c. \\
$16 \%$ in $\tilde{T}_{1} \tilde{T}_{1}$ & $17 \%$ in $\tilde{T}_{1} \overline{\tilde{T}}_{1}$ & $6 \%$ in $B_{1} \bar{B}_{5}$ and c.c. \\
$5 \%$ in $t \bar{t}$ & $12 \%$ in $T_{2} \bar{T}_{2}$ & $5 \%$ in $t \bar{T}_{4}$ and c.c. \\
$4 \%$ in $B_{1} \bar{B}_{2}$ and c.c. & $11 \%$ in $B_{1} \bar{B}_{2}$ and c.c. & $2 \%$ in $b \bar{B}_{1}$ and c.c. \\
$4 \%$ in $B_{1} \bar{B}_{1}$ & $7 \%$ in $t \bar{t}$ and $b \bar{b}$ & $1.5 \%$ in $W^{+} W_{1,2}^{-}$and c.c. \\
$2 \%$ in $B_{2,3} \bar{B}_{2,3}$ & $0.7 \%$ in $W^{+} W^{-}$and $Z^{0} H$ & $1.5 \%$ in $Z_{3} H$ \\
\hline
\end{tabular}

Table 17: BRs of the new neutral gauge bosons of the 4DCHM in the large width regime.

\begin{tabular}{|l||l|}
\hline$W_{2}^{+}$ & $W_{3}^{+}$ \\
\hline $29 \%$ in $\tilde{T}_{1} \bar{T}_{1}$ & $22 \%$ in $B_{3} \tilde{B}_{1}$ \\
$17 \%$ in $T_{1} \bar{B}_{2}$ & $22 \%$ in $T_{1} \bar{B}_{3}$ \\
$15 \%$ in $t \bar{b}$ & $15 \%$ in $B_{5} \overline{\tilde{B}}_{1}$ \\
$13 \%$ in $\overline{\tilde{B}}_{1} B_{1,2}$ & $15 \%$ in $T_{1} \bar{B}_{5}$ \\
$10 \%$ in $T_{1} \bar{B}_{1}$ & $9 \%$ in $\tilde{T}_{1} \bar{t}$ \\
$0.7 \%$ in $W^{+} Z^{0}$ and $W^{+} H$ & $6 \%$ in $\tilde{T}_{2} \bar{t}$ \\
& $2 \%$ in $t \bar{B}_{4}$ \\
& $1 \%$ in $b \overline{\tilde{B}}_{1}$ and $T_{1} \bar{b}$ \\
& $1 \%$ in $W_{1,2}^{+} Z^{0}$ and $W^{+} Z_{3}$ \\
& $1 \%$ in $W_{1}^{+} H$ \\
\hline
\end{tabular}

Table 18: BRs of the new charged gauge bosons of the 4DCHM in the large width regime.

\subsection{1.d The role of the Higgs mass}

Before investigating the phenomenology of $Z^{\prime}$ and $W^{\prime}$ production and decay in DY processes, we would like to show explicitly the impact that the recent LHC (and Tevatron) data on a possible Higgs boson have on the 4DCHM, specifically on its fermionic sector. This is mandatory, if one recalls that Ref. [9] predates such data.

We therefore present in Fig. 5 the allowed masses of the lightest additional fermion of charge $+2 / 3$ in the window $115 \mathrm{GeV} \leq m_{H} \leq 135 \mathrm{GeV}$, for the choice $f=1.2 \mathrm{TeV}$ and $g_{*}=1.8$. (The pattern for the counterpart with charge $-1 / 3$ is rather similar.) As already realised in Ref. [9], such a small Higgs mass is consistent with light fermionic partners, down to $400 \mathrm{GeV}$ or so (hence well within the scope of the LHC). In fact, the 


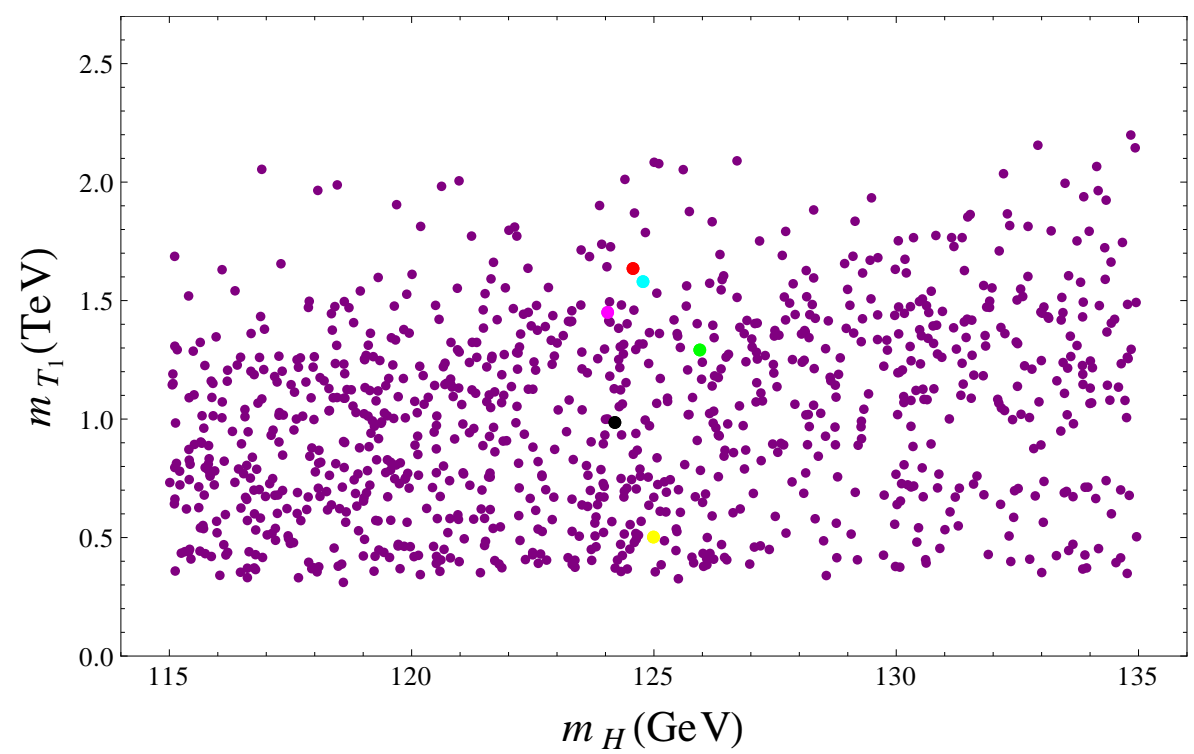

Figure 5: Mass of ligthest additional fermion of charge $+2 / 3$ versus the mass of the Higgs boson of the 4DCHM, for the choice $f=1.2 \mathrm{TeV}$ and $g_{*}=1.8$. The fermionic parameters are varied between 0.5 and $5 \mathrm{TeV}$, except $\Delta_{b L}$ and $\Delta_{b R}$ that are varied between 0.05 and $0.5 \mathrm{TeV}$. Amongst the majority of purple points, we singled out six differently coloured ones, in (1) red, (2) green, (3) cyan, (4) magenta, (5) black and (6) yellow, which correspond to benchmarks that will be used in the forthcoming collider study.

plot confirms the well established result within the 4DCHM that a light Higgs boson prefers light fermionic partners [45, 18], thereby further corroborating our claim of a strong interplay that should be expected in DY processes between the gauge and fermionic sectors of the 4DCHM.

We also use this plot to highlight some benchmark configurations. Amongst the generic points we have highlighted six in particular, differently coloured, that will define a convention that will be exploited in the upcoming phenomenological analysis. Their use will be as follows.

- The points in red, green, cyan, magenta, black and yellow correspond to the benchmarks studied in Figs. 16 17, in which we will maintain the same colour scheme. The exact numerical values of the parameters defining these are found in Tab. 19.

- Amongst these, the points in red, black and yellow correspond to the small, medium and large width benchmarks of the previous subsections.

- In particular, the point in red corresponds to the benchmark investigated in detail in Figs. 1213.

\subsubsection{Spectrum with $f=0.8 \mathrm{TeV}$ and $g_{*}=2.5$ (and the other benchmarks)}

This section is introduced merely to confirm that the presence of the width distributions as seen in the case $f=1.2 \mathrm{TeV}$ and $g_{*}=1.8$ is repeated also for the most extreme benchmark point, namely, $f=0.8 \mathrm{TeV}$ and $g_{*}=2.5$, in the sense that this choice 
(amongst all those adopted, see (a)-(f) above), is the one for which the typical mass difference between gauge bosons (governed by the parameter $g_{*}$ ) and heavy fermions (governed by $f$ ) is maximal. Even here, there exist the two populations (i)-(ii) specified above, though limited to the case of the lightest states $Z_{2}, Z_{3}$ and $W_{2}$ (the only accessible ones at the LHC in fact, see below), i.e., not for the case of the heaviest states $Z_{5}$ and $W_{3}$. Further, population (i) is very sparse while population (ii) does not obviously show the sudden rise in width size seen above, due to the opening of additional $F \bar{F}$ channels and/or the onsetting of phase space effects onto pre-existing ones. All this is clearly exemplified in Figs. 6and 7. Finally, although not shown, we can also confirm that the typical decay patterns of all the $Z^{\prime}$ and $W^{\prime}$ gauge bosons of the 4DCHM seen here is consistent with the results of the previous sub-sections, so we refrain here from presenting the corresponding results.

For all other benchmarks studied, i.e., (a), (c), (d) and (e), the $Z^{\prime}$ and $W^{\prime}$ decay dynamics is somewhere in between the cases (b) and (f), yet displaying the same unmistakable characteristics that we have just discussed. The numerical values of the input and output parameters of the 4DCHM used for the phenomenological analysis are found in Tab. 20, which will refer to Figs. 811 .

\section{Phenomenology of $Z^{\prime}$ and $W^{\prime}$ production and decay}

We consider the two tree-level processes

$$
p p \rightarrow l^{+} l^{-}
$$

and

$$
p p \rightarrow l^{+} \nu_{l}+\text { c.c. }
$$

where $l=e$ or $\mu$. The initial state in both cases includes all possible quark-antiquark subchannels, the latter being dominated (especially at large invariant masses) by contributions involving valence quark: ${ }^{13}$.

Notice that in the forthcoming analysis we will present the rates for either of the lepton flavours, not the sum of the two. This will enable us to discuss mass reconstruction efficiencies (resolutions) separately. The latter in fact change significantly from electron to muons. Considering the $\mathrm{NC}, e^{+} e^{-}$is much better than $\mu^{+} \mu^{-}$, as the mass resolution for the former is about $1 \%$ while for the latter is about $10 \%$, assuming a resonance at $2 \mathrm{TeV}$ or so (the typical lightest mass of our benchmarks). Here, the mass concerned, reconstructing the $Z^{\prime}$ resonances, is the invariant one, $M_{l^{+} l^{-}} \equiv \sqrt{\left(p_{l^{+}}+p_{l^{-}}\right)^{2}}$. In the case of the CC, the mass resolution is typically $20 \%$ for both and is dominated by the uncertainty in reconstructing the missing transverse energy/momentum (due to the neutrino escaping detection). Here, the mass concerned, reconstructing the $W^{\prime}$ resonances, is the transverse one, $M_{T} \equiv \sqrt{\left(E_{l}^{T}+E_{\text {miss }}^{T}\right)^{2}-\left(p_{l}^{x}+p_{\text {miss }}^{x}\right)^{2}-\left(p_{l}^{y}+p_{\text {miss }}^{y}\right)^{2}}$, where $E^{T}$ represents missing energy/momentum (as we consider the electron and muon massless) in the transverse plane and $p_{x, y}$ are the two components therein (assuming that the proton beams are directed along the $z$ axis). Finally, notice that the upcoming plots we will use the benchmarks defined in the previous Section, i.e., the set (a)-(f) of Tab. 20 and the set

\footnotetext{
${ }^{13}$ In particular, the $b \bar{b}$ contribution in the NC case amounts to the percent level.
} 

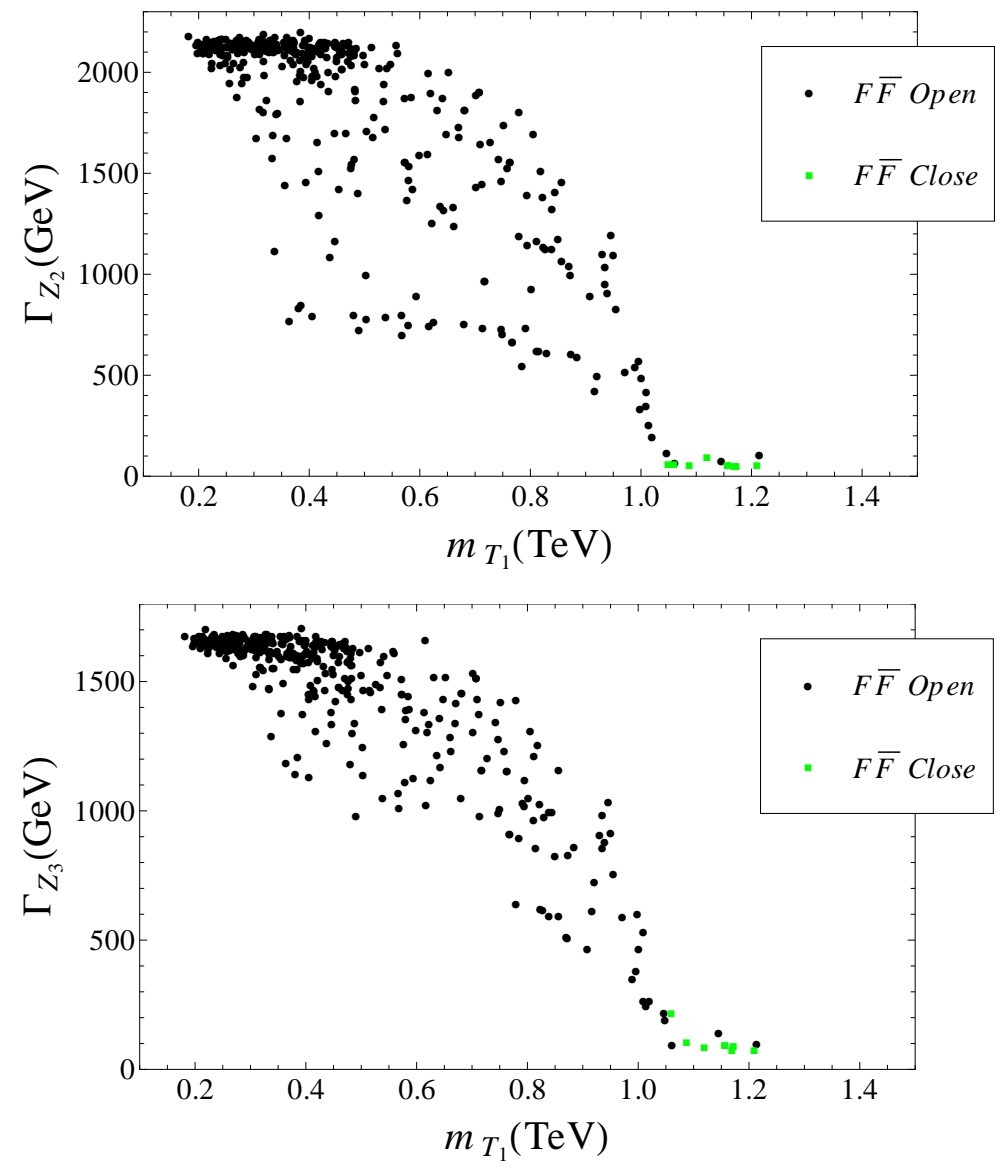

(a)

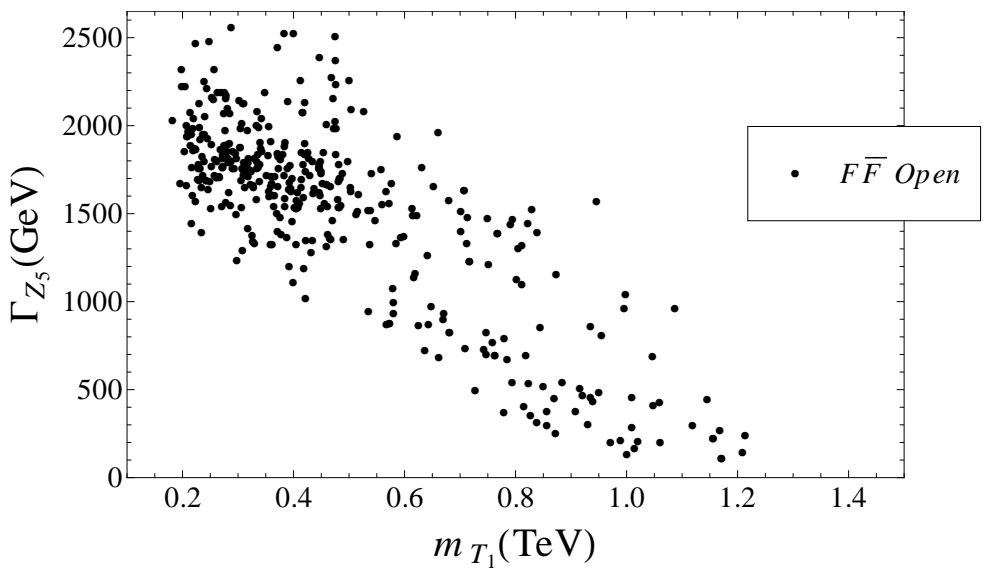

(b)

(c)

Figure 6: Width of the additional neutral gauge bosons of the 4DCHM, for the choice $f=0.8 \mathrm{TeV}$ and $g_{*}=2.5$, (a) for $Z_{2}$ (b) for $Z_{3}$ and (c) for $Z_{5}$, as a function of the mass of the lightest fermionic resonance of charge $+2 / 3$. The circle points in black are the ones where the decay in a pair of heavy fermions is permitted while the square points in green are the ones where this process is forbidden. The fermionic parameters are varied between 0.5 and $5 \mathrm{TeV}$, except $\Delta_{b L}$ and $\Delta_{b R}$ that are varied between 0.05 and $0.5 \mathrm{TeV}$. 

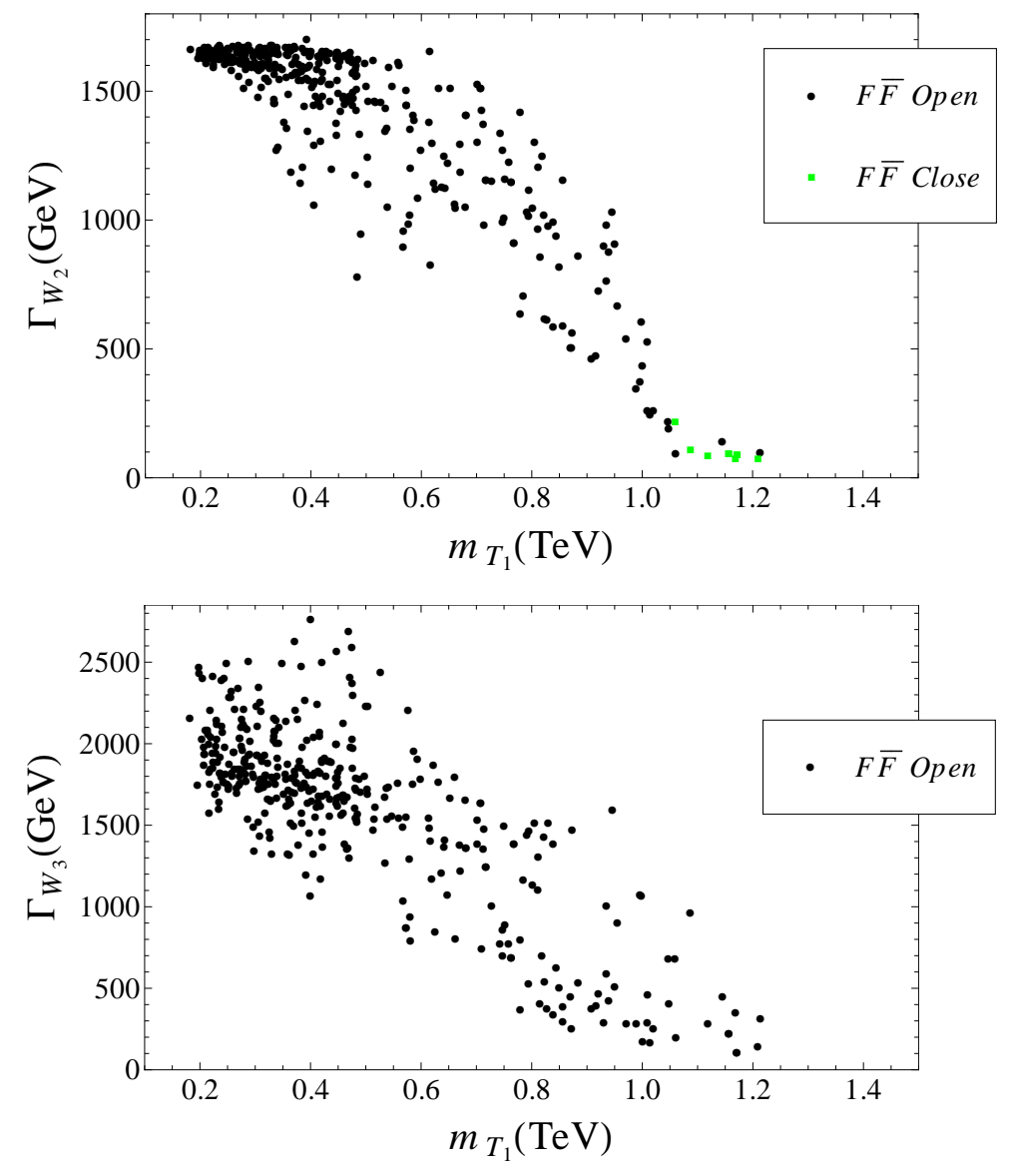

(a)

Figure 7: Width of the additional charged gauge bosons of the 4DCHM, for the choice $f=0.8 \mathrm{TeV}$ and $g_{*}=2.5$, (a) for $W_{2}$ and (b) for $W_{3}$, as a function of the mass of the lightest fermionic resonance of charge $+2 / 3$. The circle points in black are the ones where the decay in a pair of heavy fermions is permitted while the square points in green are the ones where this process is forbidden. The fermionic parameters are varied between 0.5 and $5 \mathrm{TeV}$, except $\Delta_{b L}$ and $\Delta_{b R}$ that are varied between 0.05 and $0.5 \mathrm{TeV}$. 
(1) red

(2) green

\begin{tabular}{|c|l|l|l|c|l|l|l|}
\hline $\mathrm{f}(\mathrm{GeV})$ & 1200 & $m_{*}(\mathrm{GeV})$ & 2219 & $\mathrm{f}(\mathrm{GeV})$ & 1200 & $m_{*}(\mathrm{GeV})$ & 2014 \\
$g_{*}$ & 1.8 & $\Delta_{t_{L}}(\mathrm{GeV})$ & 2366 & $g_{*}$ & 1.8 & $\Delta_{t_{L}}(\mathrm{GeV})$ & 3303 \\
$g_{0}$ & 0.69 & $\Delta_{t_{R}}(\mathrm{GeV})$ & 2245 & $g_{0}$ & 0.69 & $\Delta_{t_{R}}(\mathrm{GeV})$ & 3158 \\
$g_{0 Y}$ & 0.37 & $Y_{T}(\mathrm{GeV})$ & 2824 & $g_{0 Y}$ & 0.37 & $Y_{T}(\mathrm{GeV})$ & 1907 \\
$\langle h\rangle(\mathrm{GeV})$ & 248 & $M_{Y_{T}}(\mathrm{GeV})$ & -1043 & $\langle h\rangle(\mathrm{GeV})$ & 248 & $M_{Y_{T}}(\mathrm{GeV})$ & -647 \\
\cline { 5 - 8 } & $\Delta_{b_{L}}(\mathrm{GeV})$ & 202 & & & $\Delta_{b_{L}}(\mathrm{GeV})$ & 493 \\
& & $\Delta_{b_{R}}(\mathrm{GeV})$ & 284 & & & $\Delta_{b_{R}}(\mathrm{GeV})$ & 366 \\
& & $Y_{B}(\mathrm{GeV})$ & 2543 & & & $Y_{B}(\mathrm{GeV})$ & 1126 \\
& & $M_{Y_{B}}(\mathrm{GeV})$ & -1378 & & & $M_{Y_{B}}(\mathrm{GeV})$ & -1884 \\
& $m_{H}(\mathrm{GeV})$ & 125 & & & $m_{H}(\mathrm{GeV})$ & 126 \\
\hline
\end{tabular}

(3) cyan

(4) magenta

\begin{tabular}{|c|c|c|c|c|c|c|c|}
\hline $\mathrm{f}(\mathrm{GeV})$ & 1200 & $m_{*}(\mathrm{GeV})$ & 1908 & $\mathrm{f}(\mathrm{GeV})$ & 1200 & $m_{*}(\mathrm{GeV})$ & 2031 \\
\hline$g_{*}$ & 1.8 & $\Delta_{t_{L}}(\mathrm{GeV})$ & 3328 & $g_{*}$ & 1.8 & $\Delta_{t_{L}}(\mathrm{GeV})$ & 4423 \\
\hline$g_{0}$ & 0.69 & $\Delta_{t_{R}}(\mathrm{GeV})$ & 4585 & $g_{0}$ & 0.69 & $\Delta_{t_{R}}(\mathrm{GeV})$ & 4419 \\
\hline & 0.37 & $Y_{T}(\mathrm{GeV})$ & 1762 & $g_{0 Y}$ & 0.37 & $Y_{T}(\mathrm{GeV})$ & 1636 \\
\hline$\langle h\rangle(\mathrm{GeV})$ & 248 & $M_{Y_{T}}(\mathrm{GeV})$ & -715 & $\langle h\rangle(\mathrm{GeV})$ & 248 & $M_{Y_{T}}(\mathrm{GeV})$ & -558 \\
\hline & & $\Delta_{b_{L}}(\mathrm{GeV})$ & 340 & & & $\Delta_{b_{L}}(\mathrm{GeV})$ & 127 \\
\hline & & $\Delta_{b_{R}}(\mathrm{GeV})$ & 414 & & & $\Delta_{b_{R}}(\mathrm{GeV})$ & 286 \\
\hline & & $Y_{B}(\mathrm{GeV})$ & 999 & & & $Y_{B}(\mathrm{GeV})$ & 4543 \\
\hline & & $M_{Y_{B}}(\mathrm{GeV})$ & -725 & & & $M_{Y_{B}}(\mathrm{GeV})$ & -1394 \\
\hline & & $m_{H}(\mathrm{GeV})$ & 125 & & & $m_{H}(\mathrm{GeV})$ & 124 \\
\hline
\end{tabular}

(5) black

(6) yellow

\begin{tabular}{|c|l|l|l|c|l|l|l|}
\hline $\mathrm{f}(\mathrm{GeV})$ & 1200 & $m_{*}(\mathrm{GeV})$ & 2216 & $\mathrm{f}(\mathrm{GeV})$ & 1200 & $m_{*}(\mathrm{GeV})$ & 1293 \\
$g_{*}$ & 1.8 & $\Delta_{t_{L}}(\mathrm{GeV})$ & 2434 & $g_{*}$ & 1.8 & $\Delta_{t_{L}}(\mathrm{GeV})$ & 4714 \\
$g_{0}$ & 0.70 & $\Delta_{t_{R}}(\mathrm{GeV})$ & 2362 & $g_{0}$ & 0.70 & $\Delta_{t_{R}}(\mathrm{GeV})$ & 3402 \\
$g_{0 Y}$ & 0.37 & $Y_{T}(\mathrm{GeV})$ & 2771 & $g_{0 Y}$ & 0.37 & $Y_{T}(\mathrm{GeV})$ & 4165 \\
$\langle h\rangle(\mathrm{GeV})$ & 248 & $M_{Y_{T}}(\mathrm{GeV})$ & -1031 & $\langle h\rangle(\mathrm{GeV})$ & 248 & $M_{Y_{T}}(\mathrm{GeV})$ & -1503 \\
\cline { 5 - 8 } & & $\Delta_{b_{L}}(\mathrm{GeV})$ & 327 & & & $\Delta_{b_{L}}(\mathrm{GeV})$ & 224 \\
& $\Delta_{b_{R}}(\mathrm{GeV})$ & 299 & & & $\Delta_{b_{R}}(\mathrm{GeV})$ & 480 \\
& & $Y_{B}(\mathrm{GeV})$ & 2815 & & & $Y_{B}(\mathrm{GeV})$ & 4260 \\
& & $M_{Y_{B}}(\mathrm{GeV})$ & -4093 & & & $M_{Y_{B}}(\mathrm{GeV})$ & -2835 \\
& $m_{H}(\mathrm{GeV})$ & 124 & & & $m_{H}(\mathrm{GeV})$ & 125 \\
\hline
\end{tabular}

Table 19: Benchmark points for the generation of Figs. 16 and 17. All points assume $f=1.2 \mathrm{TeV}$ and $g_{*}=1.8$. Figs. 18 and 19 are generated only with the benchmark (4) magenta. The numbering and colour scheme is as in Fig. 5 .

(1)-(6) of Tab. 19 which correspond to the gauge boson masses and widths of Tabs. 21 and 22 , respectively.

Fig. 8 shows the invariant mass distribution of the $\mathrm{NC}$ cross section for our six choices of the $f$ and $g_{*}$ parameters, as detailed in the caption.

While the two lightest $Z^{\prime}$ resonances, $Z_{2}$ and $Z_{3}$, are clearly accessible, this is obviously 
$\begin{array}{ll}\text { (a) } f=0.75 \mathrm{TeV} \text { and } g_{*}=2 & \text { (b) } f=0.8 \mathrm{TeV} \text { and } g_{*}=2.5\end{array}$

\begin{tabular}{|c|c|c|c|c|c|c|c|}
\hline $\mathrm{f}(\mathrm{GeV})$ & 750 & $m_{*}(\mathrm{GeV})$ & 1673 & $\mathrm{f}(\mathrm{GeV})$ & 800 & $m_{*}(\mathrm{GeV})$ & 1700 \\
\hline$g_{*}$ & 2 & $\Delta_{t_{L}}(\mathrm{GeV})$ & 974 & $g_{*}$ & 2.5 & $\Delta_{t_{L}}(\mathrm{GeV})$ & 1225 \\
\hline$g_{0}$ & 0.68 & $\Delta_{t_{R}}(\mathrm{GeV})$ & 1780 & $g_{0}$ & 0.67 & $\Delta_{t_{R}}(\mathrm{GeV})$ & 1391 \\
\hline$g_{0 Y}$ & 0.37 & $Y_{T}(\mathrm{GeV})$ & 2442 & $g_{0 Y}$ & 0.36 & $Y_{T}(\mathrm{GeV})$ & 2770 \\
\hline$\langle h\rangle(\mathrm{GeV})$ & 251 & $M_{Y_{T}}(\mathrm{GeV})$ & -1231 & $\langle h\rangle(\mathrm{GeV})$ & 250 & $M_{Y_{T}}(\mathrm{GeV})$ & -1339 \\
\hline & & $\Delta_{b_{L}}(\mathrm{GeV})$ & & & & $\Delta_{b_{L}}(\mathrm{GeV})$ & 222 \\
\hline & & $\Delta_{b_{R}}(\mathrm{GeV})$ & 238 & & & $\Delta_{b_{R}}(\mathrm{GeV})$ & 99 \\
\hline & & $Y_{B}(\mathrm{GeV})$ & 2884 & & & $Y_{B}(\mathrm{GeV})$ & 2485 \\
\hline & & $M_{Y_{B}}(\mathrm{GeV})$ & -1878 & & & $M_{Y_{B}}(\mathrm{GeV})$ & -1185 \\
\hline & & $m_{H}(\mathrm{GeV})$ & 126 & & & $m_{H}(\mathrm{GeV})$ & 125 \\
\hline
\end{tabular}

(c) $f=1 \mathrm{TeV}$ and $g_{*}=2$

(d) $f=1 \mathrm{TeV}$ and $g_{*}=2.5$

\begin{tabular}{|c|l|l|l|}
\hline $\mathrm{f}(\mathrm{GeV})$ & 1000 & $m_{*}(\mathrm{GeV})$ & 1915 \\
$g_{*}$ & 2 & $\Delta_{t_{L}}(\mathrm{GeV})$ & 1503 \\
$g_{0}$ & 0.69 & $\Delta_{t_{R}}(\mathrm{GeV})$ & 1972 \\
$g_{0 Y}$ & 0.37 & $Y_{T}(\mathrm{GeV})$ & 2901 \\
$\langle h\rangle(\mathrm{GeV})$ & 249 & $M_{Y_{T}}(\mathrm{GeV})$ & -1303 \\
& $\Delta_{b_{L}}(\mathrm{GeV})$ & 196 \\
& $\Delta_{b_{R}}(\mathrm{GeV})$ & 187 \\
& $Y_{B}(\mathrm{GeV})$ & 2662 \\
& $M_{Y_{B}}(\mathrm{GeV})$ & -984 \\
& $m_{H}(\mathrm{GeV})$ & 126 \\
\hline
\end{tabular}

\begin{tabular}{|c|l|l|l|}
\hline $\mathrm{f}(\mathrm{GeV})$ & 1000 & $m_{*}(\mathrm{GeV})$ & 2027 \\
$g_{*}$ & 2.5 & $\Delta_{t_{L}}(\mathrm{GeV})$ & 1677 \\
$g_{0}$ & 0.67 & $\Delta_{t_{R}}(\mathrm{GeV})$ & 2209 \\
$g_{0 Y}$ & 0.36 & $Y_{T}(\mathrm{GeV})$ & 2578 \\
$\langle h\rangle(\mathrm{GeV})$ & 249 & $M_{Y_{T}}(\mathrm{GeV})$ & -1146 \\
& & $\Delta_{b_{L}}(\mathrm{GeV})$ & 223 \\
& $\Delta_{b_{R}}(\mathrm{GeV})$ & 299 \\
& $Y_{B}(\mathrm{GeV})$ & 2319 \\
& $M_{Y_{B}}(\mathrm{GeV})$ & -1104 \\
& $m_{H}(\mathrm{GeV})$ & 125 \\
\hline
\end{tabular}

(e) $f=1.1 \mathrm{TeV}$ and $g_{*}=1.8$

(f) $f=1.2 \mathrm{TeV}$ and $g_{*}=1.8$

\begin{tabular}{|c|l|l|l|c|l|l|l|}
\hline $\mathrm{f}(\mathrm{GeV})$ & 1100 & $m_{*}(\mathrm{GeV})$ & 2318 & $\mathrm{f}(\mathrm{GeV})$ & 1200 & $m_{*}(\mathrm{GeV})$ & 2219 \\
$g_{*}$ & 1.8 & $\Delta_{t_{L}}(\mathrm{GeV})$ & 2440 & $g_{*}$ & 1.8 & $\Delta_{t_{L}}(\mathrm{GeV})$ & 2366 \\
$g_{0}$ & 0.69 & $\Delta_{t_{R}}(\mathrm{GeV})$ & 1877 & $g_{0}$ & 0.69 & $\Delta_{t_{R}}(\mathrm{GeV})$ & 2245 \\
$g_{0 Y}$ & 0.37 & $Y_{T}(\mathrm{GeV})$ & 2909 & $g_{0 Y}$ & 0.37 & $Y_{T}(\mathrm{GeV})$ & 2824 \\
$\langle h\rangle(\mathrm{GeV})$ & 248 & $M_{Y_{T}}(\mathrm{GeV})$ & -636 & $\langle h\rangle(\mathrm{GeV})$ & 248 & $M_{Y_{T}}(\mathrm{GeV})$ & -1043 \\
\cline { 5 - 8 } & & $\Delta_{b_{L}}(\mathrm{GeV})$ & 272 & & & $\Delta_{b_{L}}(\mathrm{GeV})$ & 202 \\
& $\Delta_{b_{R}}(\mathrm{GeV})$ & 208 & & & $\Delta_{b_{R}}(\mathrm{GeV})$ & 284 \\
& & $Y_{B}(\mathrm{GeV})$ & 2435 & & & $Y_{B}(\mathrm{GeV})$ & 2543 \\
& & $M_{Y_{B}}(\mathrm{GeV})$ & -1429 & & & $M_{Y_{B}}(\mathrm{GeV})$ & -1378 \\
& & $m_{H}(\mathrm{GeV})$ & 124 & & & $m_{H}(\mathrm{GeV})$ & 125 \\
\hline
\end{tabular}

Table 20: Benchmark points for the generation of Figs. 8, 9, 10 and 11. Figs. 12 and 13 are generated only with benchmark (f). The labelling of the benchmarks is as described in the text.

not the case for the third one, $Z_{5}$, which is much suppressed to remain most probably invisible at the $14 \mathrm{TeV}$ LHC, even with a $\mathcal{O}\left(300 \mathrm{fb}^{-1}\right)$ luminosity ${ }^{14}$. In each plot we also show the SM yield over the same mass range. Standard acceptance and selection cuts

\footnotetext{
${ }^{14}$ Its presence could however be ascertained at the so-called Super-LHC, an LHC upgrade involving a tenfold increase in luminosity [46]. So is the case for the $W_{3}$ state (see further on).
} 
(a) $f=0.75 \mathrm{TeV}$ and $g_{*}=2$

(b) $f=0.8 \mathrm{TeV}$ and $g_{*}=2.5$

\begin{tabular}{|c|c|c|c|c|c|}
\hline & $M(\mathrm{GeV})$ & $\Gamma(\mathrm{GeV})$ & & $M(\mathrm{GeV})$ & $\Gamma(\mathrm{GeV})$ \\
\hline$Z_{2}$ & 1549 & 28 & $Z_{2}$ & 2041 & 61 \\
\hline$Z_{3}$ & 1581 & 26 & $Z_{3}$ & 2068 & 98 \\
\hline$Z_{5}$ & 2124 & 34 & $Z_{5}$ & 2830 & 223 \\
\hline$\overline{W_{2}}$ & 1581 & 26 & $W_{2}$ & 2067 & 98 \\
\hline$W_{3}$ & 2123 & 33 & $W_{3}$ & 2830 & 221 \\
\hline & $l^{+} l^{-}$ & $l^{+} \nu+$ c.c. & & $l^{+} l^{-}$ & $l^{+} \nu+$ c.c. \\
\hline$\sigma(\mathrm{fb})$ & $7.44[5.46]$ & $13.22[6.96]$ & $\sigma(\mathrm{fb})$ & $0.90[0.91]$ & $1.19[1.06]$ \\
\hline $\begin{array}{c}p_{l}^{T}(\mathrm{GeV}) \\
\left|\eta_{l}\right| \\
M_{l^{+} l^{-} / T}(\mathrm{TeV})\end{array}$ & $\begin{array}{l}>20 \\
<2.5 \\
>1\end{array}$ & $\begin{array}{l}>20 \\
<2.5 \\
>1\end{array}$ & $\begin{array}{c}p_{l}^{T}(\mathrm{GeV}) \\
\left|\eta_{l}\right| \\
M_{l^{+} l^{-} / T}(\mathrm{TeV})\end{array}$ & $\begin{array}{l}>20 \\
<2.5 \\
>1.5\end{array}$ & $\begin{array}{l}>20 \\
<2.5 \\
>1.5\end{array}$ \\
\hline
\end{tabular}

(c) $f=1 \mathrm{TeV}$ and $g_{*}=2$

(d) $f=1 \mathrm{TeV}$ and $g_{*}=2.5$

\begin{tabular}{|c|c|c|c|c|c|}
\hline & $M(\mathrm{GeV})$ & $\Gamma(\mathrm{GeV})$ & & $M(\mathrm{GeV})$ & $\Gamma(\mathrm{GeV})$ \\
\hline$Z_{2}$ & 2066 & 39 & $Z_{2}$ & 2552 & 81 \\
\hline$Z_{3}$ & 2111 & 52 & $Z_{3}$ & 2586 & 115 \\
\hline$Z_{5}$ & 2830 & 71 & $Z_{5}$ & 3537 & 332 \\
\hline$W_{2}$ & 2111 & 52 & $W_{2}$ & 2586 & 114 \\
\hline$W_{3}$ & 2830 & 50 & $W_{3}$ & 3537 & 328 \\
\hline & " & $\overline{l l^{+} \nu+\text { c.c. }}$ & & 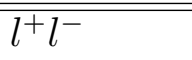 & 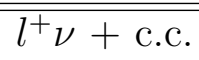 \\
\hline$\sigma(\mathrm{fb})$ & $1.24[0.91]$ & $2.04[1.06]$ & $\sigma(\mathrm{fb})$ & $0.21[0.21]$ & $0.28[0.23]$ \\
\hline $\begin{array}{c}p_{l}^{T}(\mathrm{GeV}) \\
\left|\eta_{l}\right| \\
M_{l^{+} l^{-} / T}(\mathrm{TeV})\end{array}$ & $\begin{array}{l}>20 \\
<2.5 \\
>1.5\end{array}$ & $\begin{array}{l}>20 \\
<2.5 \\
>1.5\end{array}$ & $\begin{array}{c}p_{l}^{T}(\mathrm{GeV}) \\
\left|\eta_{l}\right| \\
M_{l^{+} l^{-}} / T \\
(\mathrm{TeV})\end{array}$ & $\begin{array}{l}>20 \\
<2.5 \\
>2\end{array}$ & $\begin{array}{l}>20 \\
<2.5 \\
>2\end{array}$ \\
\hline
\end{tabular}

(e) $f=1.1 \mathrm{TeV}$ and $g_{*}=1.8$

(f) $f=1.2 \mathrm{TeV}$ and $g_{*}=1.8$

\begin{tabular}{|c|c|c|c|c|c|}
\hline & $M(\mathrm{GeV})$ & $\Gamma(\mathrm{GeV})$ & & $M(\mathrm{GeV})$ & $\Gamma(\mathrm{GeV})$ \\
\hline$Z_{2}$ & 2061 & 24 & $Z_{2}$ & 2249 & 32 \\
\hline$Z_{3}$ & 2119 & 45 & $Z_{3}$ & 2312 & 55 \\
\hline$Z_{5}$ & 2802 & 42 & $Z_{5}$ & 3056 & 54 \\
\hline$W_{2}$ & 2119 & 45 & $W_{2}$ & 2312 & 55 \\
\hline$W_{3}$ & 2802 & 42 & $W_{3}$ & 3056 & 54 \\
\hline & $l^{+} l^{-}$ & $l^{+} \nu+$ c.c. & & $l^{+} l^{-}$ & $l^{+} \nu+$ c.c. \\
\hline$\sigma(\mathrm{fb})$ & $1.37[0.21]$ & $1.33[0.23]$ & $\sigma(\mathrm{fb})$ & $0.78[0.21]$ & $1.11[0.23]$ \\
\hline $\begin{array}{c}p_{l}^{T}(\mathrm{GeV}) \\
\left|\eta_{l}\right| \\
M_{l^{+} l^{-} / T}(\mathrm{TeV})\end{array}$ & $\begin{array}{l}>20 \\
<2.5 \\
>2\end{array}$ & $\begin{array}{l}>20 \\
<2.5 \\
>2\end{array}$ & $\begin{array}{c}p_{l}^{T}(\mathrm{GeV}) \\
\left|\eta_{l}\right| \\
M_{l^{+} l^{-} / T}(\mathrm{TeV})\end{array}$ & $\begin{array}{l}>20 \\
<2.5 \\
>2\end{array}$ & $\begin{array}{l}>20 \\
<2.5 \\
>2\end{array}$ \\
\hline
\end{tabular}

Table 21: Gauge boson masses and width in GeV arising from the benchmarks of Tab. 20. The labelling of the benchmarks is as described in the text. Here, $\sigma$ represents the $4 \mathrm{DCHM}$ cross section after the cuts described herein, with the value in square brakets being the reference SM result. 
(1) red

\begin{tabular}{|l|l|l|}
\hline & $M(\mathrm{GeV})$ & $\Gamma(\mathrm{GeV})$ \\
\hline$Z_{2}$ & 2249 & 32 \\
$Z_{3}$ & 2312 & 55 \\
$Z_{5}$ & 3056 & 54 \\
\hline$W_{2}$ & 2312 & 55 \\
$W_{3}$ & 3056 & 54 \\
\hline \hline & $l^{+} l^{-}$ & $l^{+} \nu+$ c.c. \\
\hline$\sigma(\mathrm{fb})$ & 0.78 & 1.11 \\
\hline
\end{tabular}

(3) cyan
(2) green

\begin{tabular}{|l|l|l|}
\hline & $M(\mathrm{GeV})$ & $\Gamma(\mathrm{GeV})$ \\
\hline$Z_{2}$ & 2249 & 48 \\
$Z_{3}$ & 2312 & 86 \\
$Z_{5}$ & 3056 & 389 \\
\hline$W_{2}$ & 2312 & 86 \\
$W_{3}$ & 3056 & 381 \\
\hline \hline & $l^{+} l^{-}$ & $l^{+} \nu+$ c.c. \\
\hline$\sigma(\mathrm{fb})$ & 0.56 & 0.79 \\
\hline
\end{tabular}

(4) magenta

\begin{tabular}{|l|l|l||l|l|l|}
\hline & $M(\mathrm{GeV})$ & $\Gamma(\mathrm{GeV})$ & & $M(\mathrm{GeV})$ & $\Gamma(\mathrm{GeV})$ \\
\hline$Z_{2}$ & 2249 & 60 & $Z_{2}$ & 2249 & 75 \\
$Z_{3}$ & 2312 & 92 & $Z_{3}$ & 2312 & 104 \\
$Z_{5}$ & 3056 & 192 & $Z_{5}$ & 3056 & 313 \\
\hline$W_{2}$ & 2312 & 91 & $W_{2}$ & 2312 & 104 \\
$W_{3}$ & 3056 & 172 & $W_{3}$ & 3056 & 293 \\
\hline \hline & $l^{+} l^{-}$ & $l^{+} \nu+$ c.c. & & $l^{+} l^{-}$ & $l^{+} \nu+$ c.c. \\
\hline$\sigma(\mathrm{fb})$ & 0.52 & 0.76 & $\sigma(\mathrm{fb})$ & 0.47 & 0.70 \\
\hline
\end{tabular}

(5) black

(6) yellow

\begin{tabular}{|l|l|l||l|l|l|}
\hline & $M(\mathrm{GeV})$ & $\Gamma(\mathrm{GeV})$ & & $M(\mathrm{GeV})$ & $\Gamma(\mathrm{GeV})$ \\
\hline$Z_{2}$ & 2249 & 301 & $Z_{2}$ & 2249 & 1099 \\
$Z_{3}$ & 2312 & 434 & $Z_{3}$ & 2312 & 827 \\
$Z_{5}$ & 3056 & 526 & $Z_{5}$ & 3056 & 413 \\
\hline$W_{2}$ & 2312 & 434 & $W_{2}$ & 2312 & 820 \\
$W_{3}$ & 3056 & 522 & $W_{3}$ & 3056 & 614 \\
\hline \hline & $l^{+} l^{-}$ & $l^{+} \nu+$ c.c. & & $l^{+} l^{-}$ & $l^{+} \nu+$ c.c. \\
\hline$\sigma(\mathrm{fb})$ & 0.26 & 0.36 & $\sigma(\mathrm{fb})$ & 0.23 & 0.30 \\
\hline
\end{tabular}

Table 22: Gauge boson masses and widths in GeV arising from the benchmarks of Tab. 19 , All points assume $f=1.2 \mathrm{TeV}$ and $g_{*}=1.8$. The numbering and colour scheme of the benchmarks is as in Fig. 5. Here, $\sigma$ represents the 4DCHM cross section after the cuts described for benchmark (f) in Tab. 21, where also the SM reference rates can be found.

(again, as detailed in the caption) have been adopted here. In all cases a clear excess is seen around the (very close) $Z_{2}$ and $Z_{3}$ masses. While in some cases the two resonances are not resolvable, in others they can be separated, certainly in $e^{+} e^{-}$final states, but not in $\mu^{+} \mu^{-}$ones. Notice in fact that the bin width in this plots is $2 \mathrm{GeV}$, so that even assembling 10 of these (in the case of electron-positron pairs) does not spoil the ability to establish the two resonances separately, while such a separation is not possible for the case of muon-antimuon pairs (as this imply integrating over 100 bins). Pure SM event rates are very small compared to those emerging in the $4 \mathrm{DCHM}$ (the latter are the complete result, including not only the $Z^{\prime}$ resonances individually, but also their 

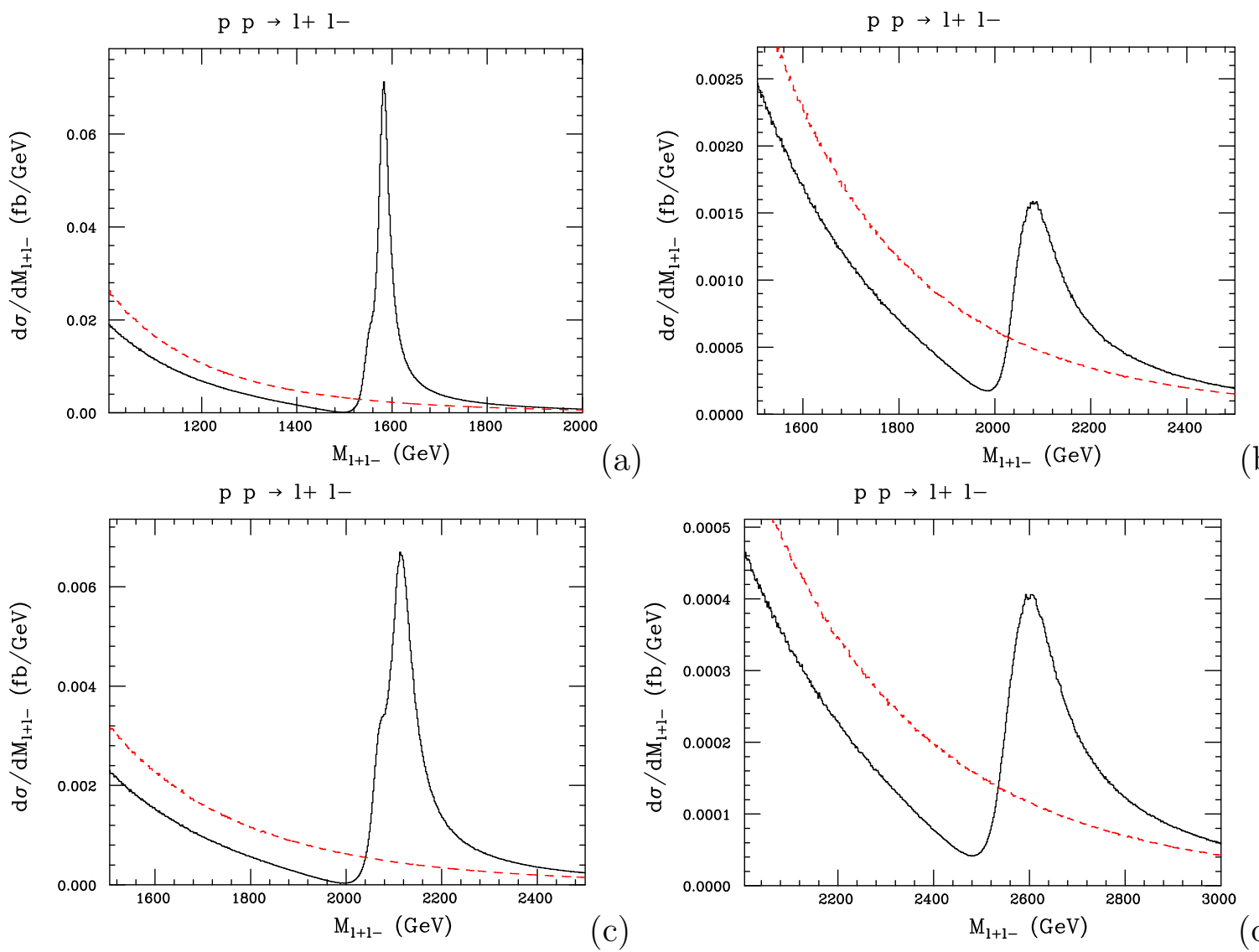

(b)
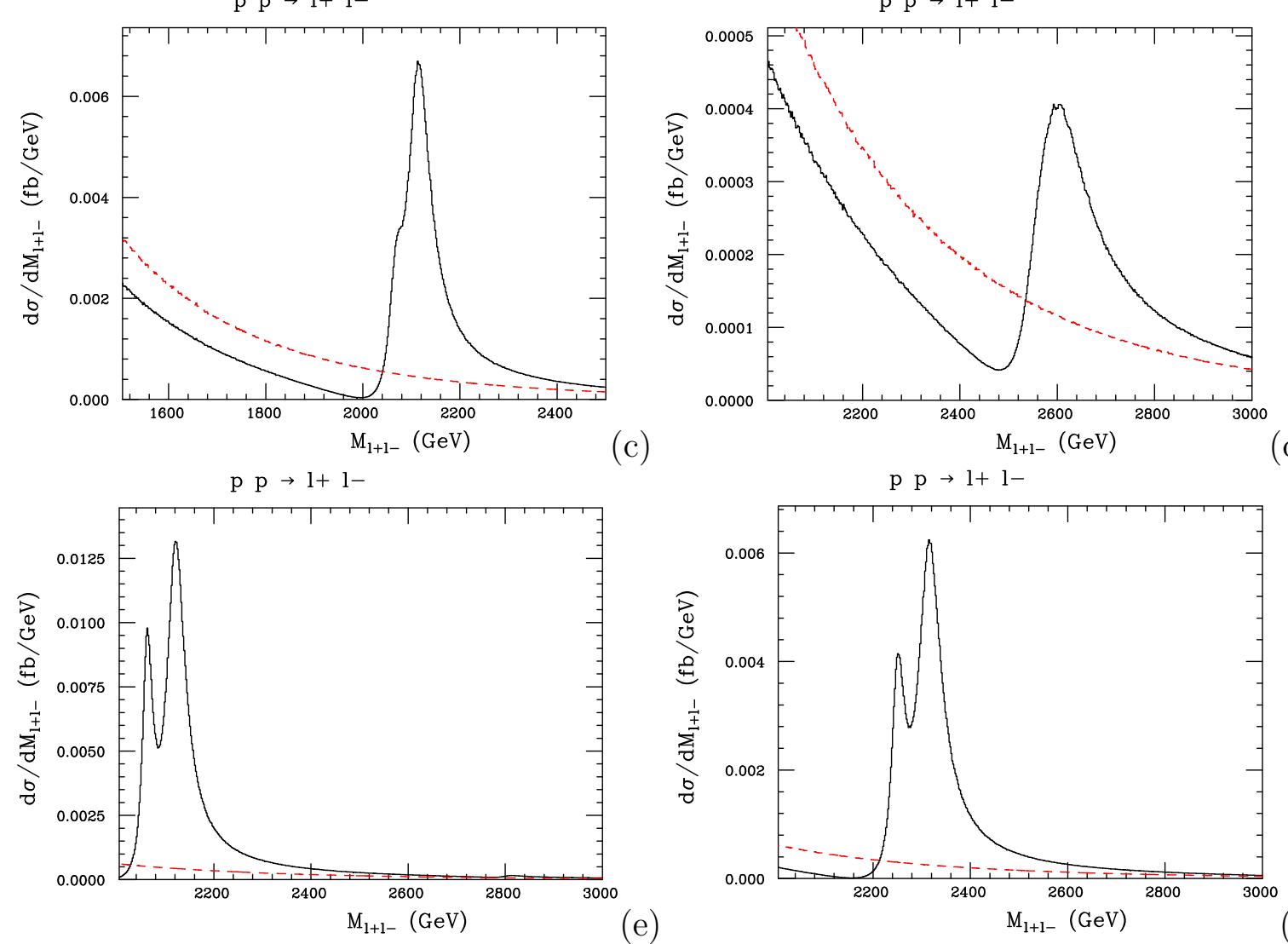

Figure 8: Differential distributions in invariant mass $M_{l^{+} l^{-}}$for the cross section at the 14 TeV LHC for NC DY in the 4DCHM (solid) for (a) $f=0.75 \mathrm{TeV}$ and $g^{*}=2$ (b) $f=0.8$ $\mathrm{TeV}$ and $g^{*}=2.5$ (c) $f=1 \mathrm{TeV}$ and $g^{*}=2$ (d) $f=1 \mathrm{TeV}$ and $g^{*}=2.5$ (e) $f=1.1$ $\mathrm{TeV}$ and $g^{*}=1.8$ (f) $f=1.2 \mathrm{TeV}$ and $g^{*}=1.8$ and in the SM (dashed). The integrated cross sections are (a) 7.44[5.46] fb (b) $0.90[0.91] \mathrm{fb}$ (c) $1.24[0.91] \mathrm{fb}$ (d) $0.21[0.21] \mathrm{fb}(\mathrm{e})$ $1.37[0.21] \mathrm{fb}(\mathrm{f}) 0.78[0.21] \mathrm{fb}$ for the $4 \mathrm{DCHM}[\mathrm{SM}]$ after the cuts $p_{l}^{T}>20 \mathrm{GeV},\left|\eta_{l}\right|<2.5$ and (a) $M_{l^{+} l^{-}}>1.0 \mathrm{TeV}$ (b) $M_{l^{+} l^{-}}>1.5 \mathrm{TeV}$ (c) $M_{l^{+} l^{-}}>1.5 \mathrm{TeV}$ (d) $M_{l^{+} l^{-}}>2.0 \mathrm{TeV}$ (e) $M_{l^{+} l^{-}}>2.0 \mathrm{TeV}$ (f) $M_{l^{+} l^{-}}>2.0 \mathrm{TeV}$. Bin width is here $2 \mathrm{GeV}$.

interferences as well as the SM contribution due to $\gamma$ and $Z^{0}$ exchange, together with the relative interferences with the resonant $Z^{\prime}$ contributions), to the extent that analyses 
should essentially be background free in several cases. Overall normalisations of the cross sections are typically of $\mathcal{O}(1 \mathrm{fb})$ to $\mathcal{O}(10 \mathrm{fb})$, thereby rendering each of these parameter configurations of the $4 \mathrm{DMCH}$ accessible at the LHC (albeit through the two lowest mass resonances only).

The corresponding case for the CC cross section is displayed in Fig. 9 (again, see the caption for parameter choices, cuts and normalisations).

Here, only two resonances are involved, i.e., $W_{2}$ and $W_{3}$, though the heaviest one is again unreachable with the foreseen standard LHC configuration. In this channel it is not possible to reconstruct the mass of the decaying $W^{\prime}$ boson from a resonance, rather one uses the transverse mass, as explained, which is much less correlated to the intervening $M_{W^{\prime}}$ value, so that the visible peaks are much broader in comparison to the NC case and also somewhat displaced towards smaller masses with respect to the true mass values of the $4 \mathrm{DMCH}$ gauge boson. Again though, in all cases, the enhancement is well visible over the SM noise. Further, event rates are somewhat larger than in the NC case.

In both the $\mathrm{NC}$ and $\mathrm{CC}$ channel, it is possible to define the AFB of the cross section, as the direction of the reference incoming quark or antiquark can be inferred from the direction of the boost onto the final state in the laboratory frame. The AFB can be sampled in invariant (NC) or transverse (CC) mass bins [47, by defining

$$
\frac{d \mathrm{AFB}}{d M}=\frac{d \sigma(\cos \theta>0) / d M-d \sigma(\cos \theta<0) / d M}{d \sigma(\cos \theta>0) / d M+d \sigma(\cos \theta<0) / d M},
$$

where $\theta$ is the polar angle of the reference outgoing lepton or antilepton relative to the direction of the reference incoming quark or antiquark ${ }^{15}$ and $M \equiv M_{l^{+} l^{-}}$for process (15) or $M \equiv M_{T}$ for process (16). Notice that in the CC case one can not reconstruct unambiguously the longitudinal component of the neutrino momentum to define the asymmetry, because of the twofold solution from the mass equation. So we take both solutions and plot each of them with half weight, which somewhat dilutes the asymmetry, in order to individuate the direction of the boost. This is done by assuming that the invariant mass of the final state coincide with the $W$ mass in the case of the SM hypothesis and with the $W_{2,3}$ mass in the case of the 4DCHM hypothesis (where an indicative value for the latter can be obtained from the NC, in the spirit of point 4 discussed in Sect. 1 . and elaborated upon later on).

Remarkably, for the NC channel, see Fig. 10, such an observable displays a peculiar dependence in the vicinity of all intermediate $Z^{\prime}$ masses, including the heavy one (i.e., $Z_{5}$ ), for all $f$ and $g_{*}$ combinations. Furthermore, these effects should be resolvable no matter the final state, as the reader should notice that this observable is sampled in the plots over histogram bins which are $50 \mathrm{GeV}$ wide. However, as already mentioned, it remains debatable whether even this observable can be used to single out the heaviest of the $Z$ "s.

The plots for AFB in the case of the CC process are found in Fig. 11. Herein, one notices that the resolving power of the resonances in the AFB is diminished, as the presence of the heaviest $W^{\prime}$ boson is hardly visible (just a little 'kink' and only in some of the cases). In this connection, further recall that the transverse mass resolution cannot afford one with exploring the yield of a smaller bin width.

\footnotetext{
${ }^{15}$ In presence of real QCD radiation, it would become more appropriate to define the polar angle in the Collins-Soper frame [4].
} 

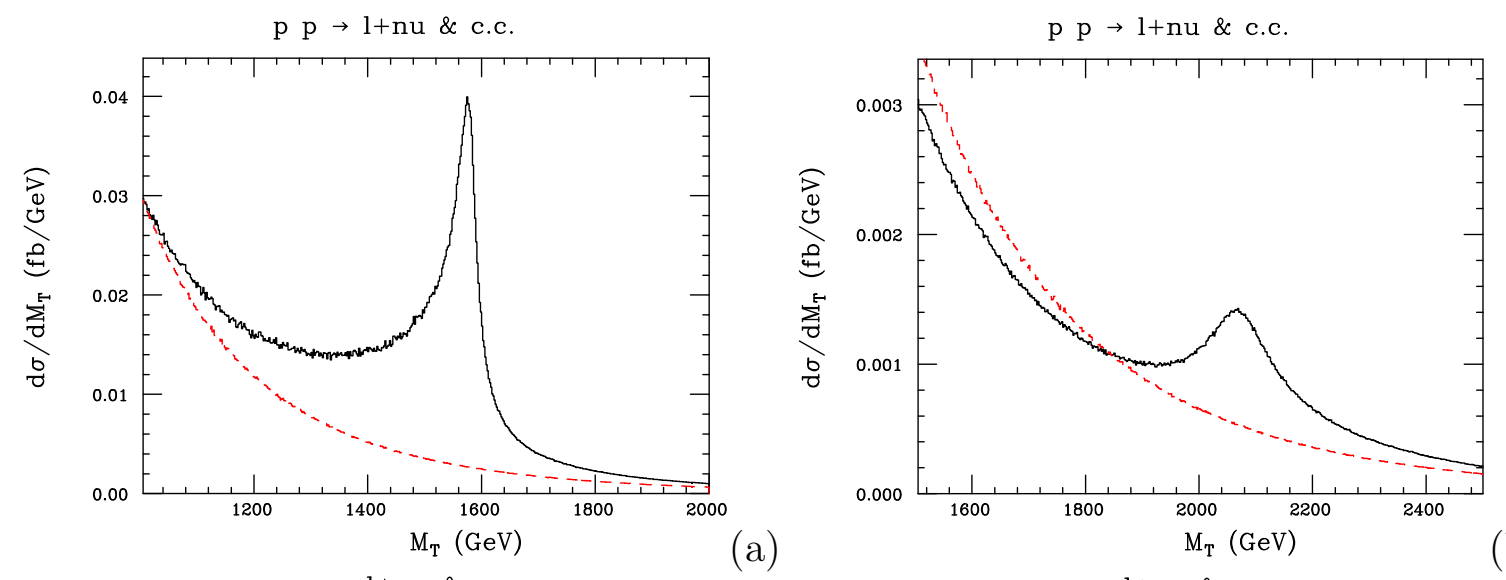

(b)
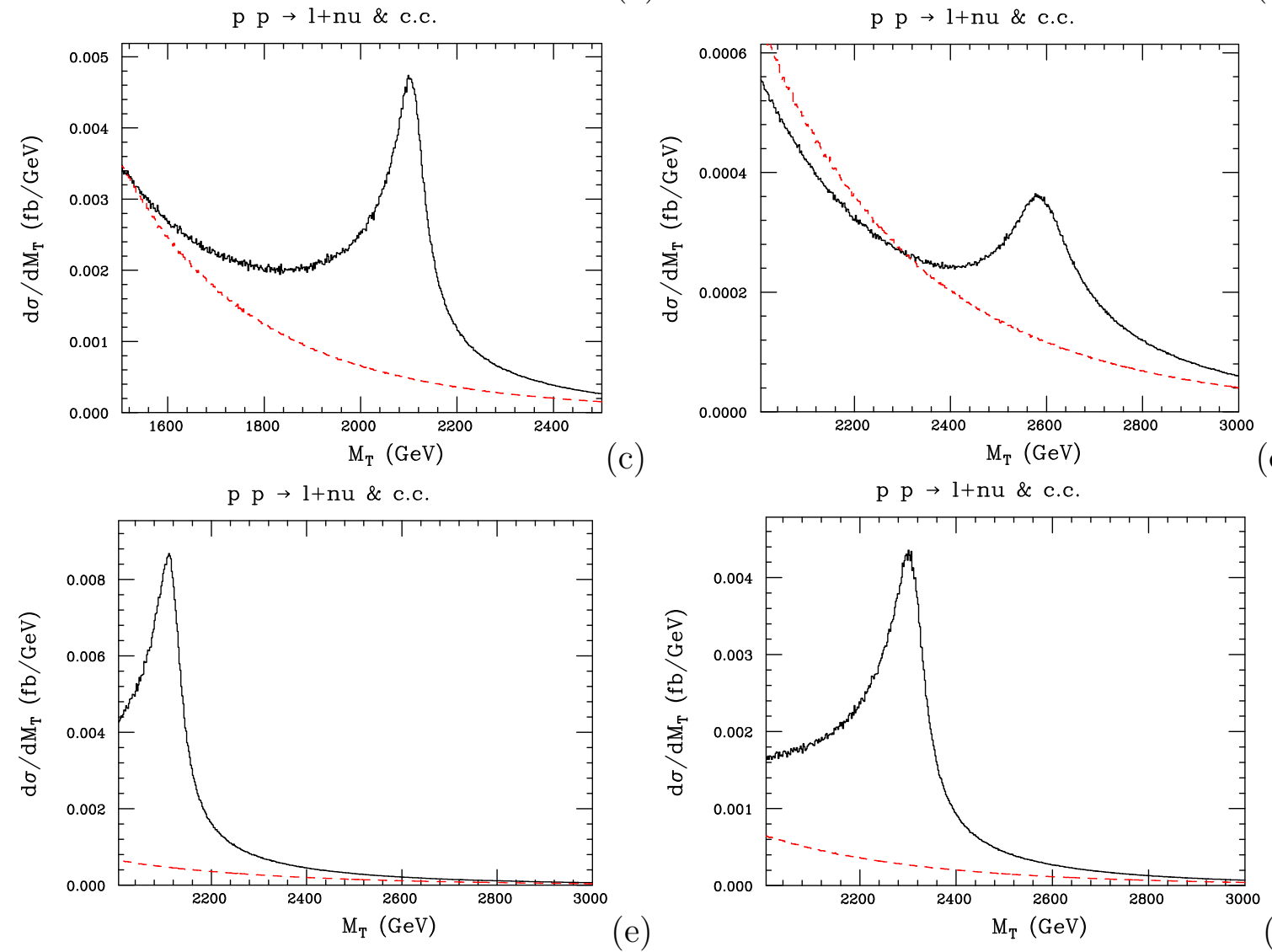

Figure 9: Differential distributions in transverse mass $M_{T}$ for the cross section at the 14 TeV LHC for CC DY in the 4DCHM (solid) for (a) $f=0.75 \mathrm{TeV}$ and $g^{*}=2.0$ (b) $f=0.8$ $\mathrm{TeV}$ and $g^{*}=2.5$ (c) $f=1 \mathrm{TeV}$ and $g^{*}=2$ (d) $f=1 \mathrm{TeV}$ and $g^{*}=2.5$ (e) $f=1.1$ $\mathrm{TeV}$ and $g^{*}=1.8$ (f) $f=1.2 \mathrm{TeV}$ and $g^{*}=1.8$ and in the SM (dashed). The integrated cross sections are (a) 13.22[6.96] fb (b) 1.19[1.06] fb (c) 2.04[1.06] fb (d) 0.28[0.23] fb (e) $1.33[0.23] \mathrm{fb}(\mathrm{f}) 1.11[0.23] \mathrm{fb}$ for the $4 \mathrm{DCHM}[\mathrm{SM}]$ after the cuts $p_{l}^{T}>20 \mathrm{GeV},\left|\eta_{l}\right|<2.5$ and (a) $M_{T}>1.0 \mathrm{TeV}$ (b) $M_{T}>1.5 \mathrm{TeV}$ (c) $M_{T}>1.5 \mathrm{TeV}$ (d) $M_{T}>2.0 \mathrm{TeV}$ (e) $M_{T}>2.0 \mathrm{TeV}$ (f) $M_{T}>2.0 \mathrm{TeV}$. Bin width is here $2 \mathrm{GeV}$.

In order to explore the composition of the 4DCHM signals, both in the $\mathrm{NC}$ and CC current, we present Figs. 12 and 13 , respectively. In both cases, we show the mass 

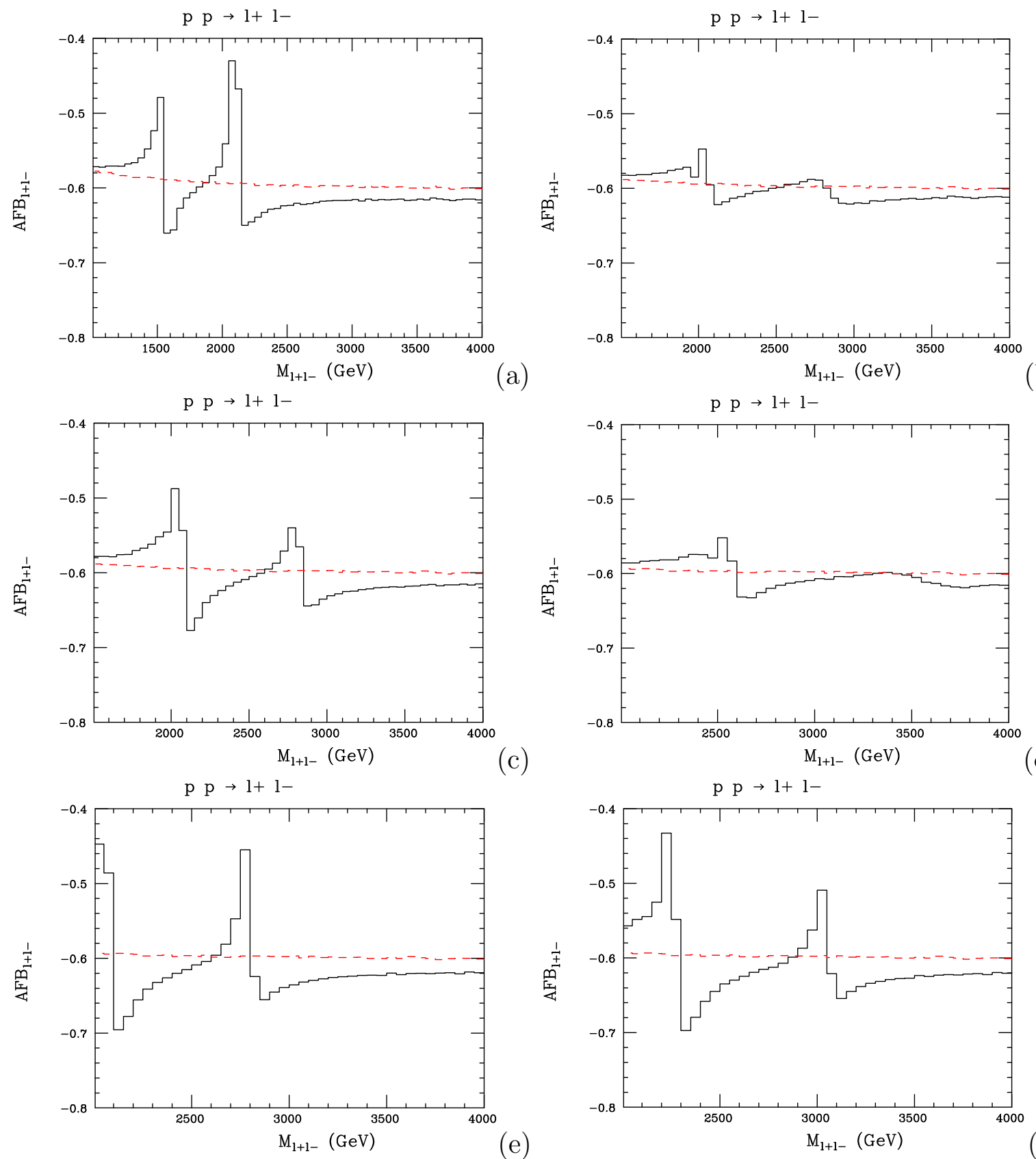

Figure 10: Differential distributions in invariant mass $M_{l^{+} l^{-}}$for the forward-backward asymmetry at the $14 \mathrm{TeV}$ LHC for NC DY in the 4DCHM (solid) for (a) $f=0.75 \mathrm{TeV}$ and $g^{*}=2$ (b) $f=0.8 \mathrm{TeV}$ and $g^{*}=2.5$ (c) $f=1 \mathrm{TeV}$ and $g^{*}=2$ (d) $f=1 \mathrm{TeV}$ and $g^{*}=2.5$ (e) $f=1.1 \mathrm{TeV}$ and $g^{*}=1.8$ (f) $f=1.2 \mathrm{TeV}$ and $g^{*}=1.8$ and in the SM (dashed). Cuts, cross sections and mass/width parameters as in the previous plots. Bin width is here $50 \mathrm{GeV}$.

dependence of the cross section and the AFB. While for both channels it is clear that the shape of the cross section (top frames) is largely due to the contribution of the resonant 4DCHM diagrams, it is interesting to notice that the shape of the AFB in the NC channel is driven by interference effects between the $4 \mathrm{DCHM}$ resonant diagrams $\left(Z^{\prime}\right)$ and the SM 

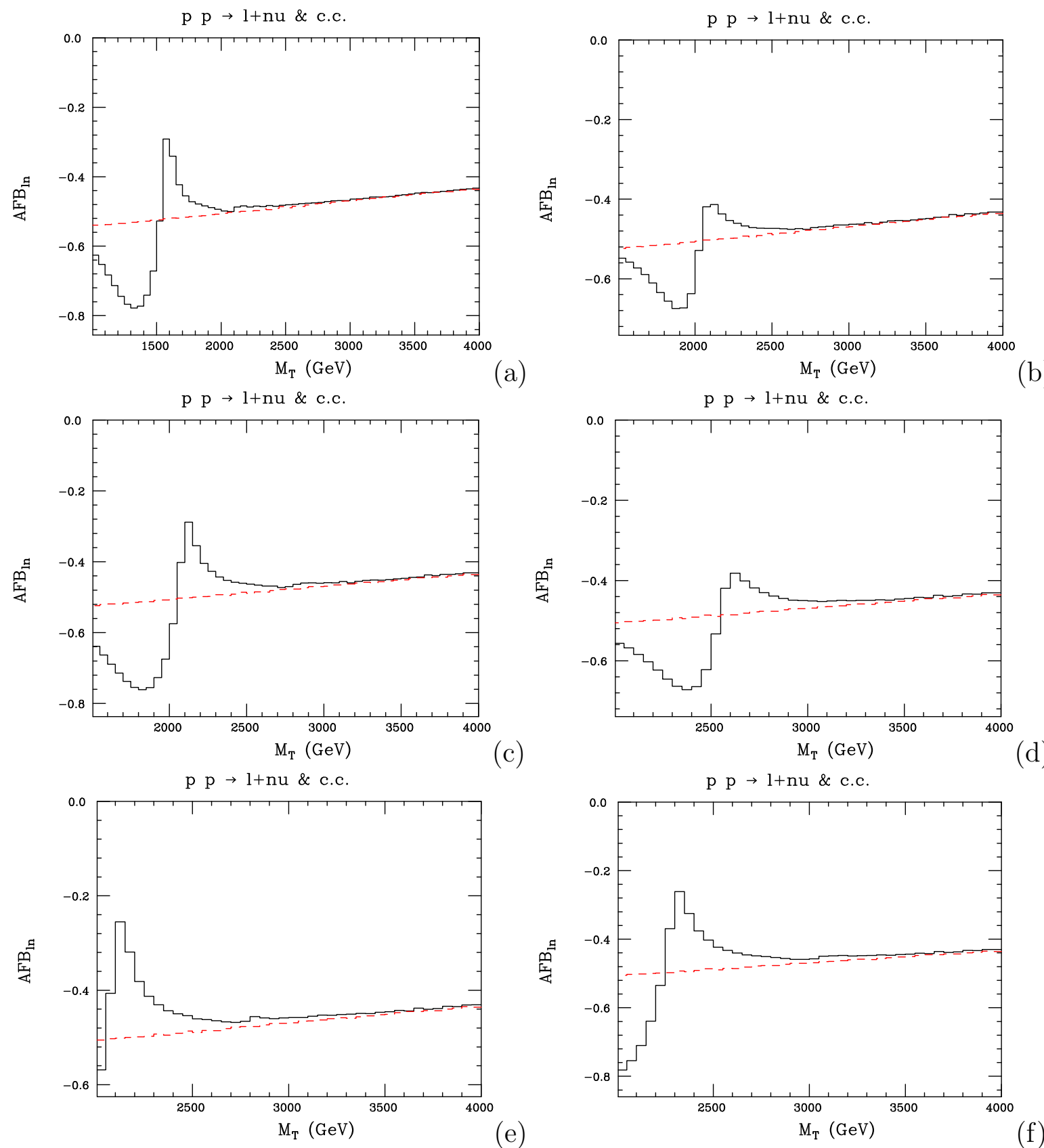

Figure 11: Differential distributions in transverse mass $M_{T}$ for the forward-backward asymmetry at the $14 \mathrm{TeV}$ LHC for CC DY in the 4DCHM (solid) for (a) $f=0.75 \mathrm{TeV}$ and $g^{*}=2$ (b) $f=0.8 \mathrm{TeV}$ and $g^{*}=2.5$ (c) $f=1 \mathrm{TeV}$ and $g^{*}=2$ (d) $f=1 \mathrm{TeV}$ and $g^{*}=2.5$ (e) $f=1.1 \mathrm{TeV}$ and $g^{*}=1.8$ (f) $f=1.2 \mathrm{TeV}$ and $g^{*}=1.8$ and in the SM (dashed). Cuts, cross sections and mass/width parameters as in the previous plots. Bin width is here $50 \mathrm{GeV}$.

contributions, while in the case of the CC process this is also due to $4 \mathrm{DCHM}$ resonances $\left(W^{\prime}\right)$.

To be able to quantitatively address the distinguishability between the 4DCHM and $\mathrm{SM}$, the statistical error of the predictions ought to be calculated. While we can confirm 

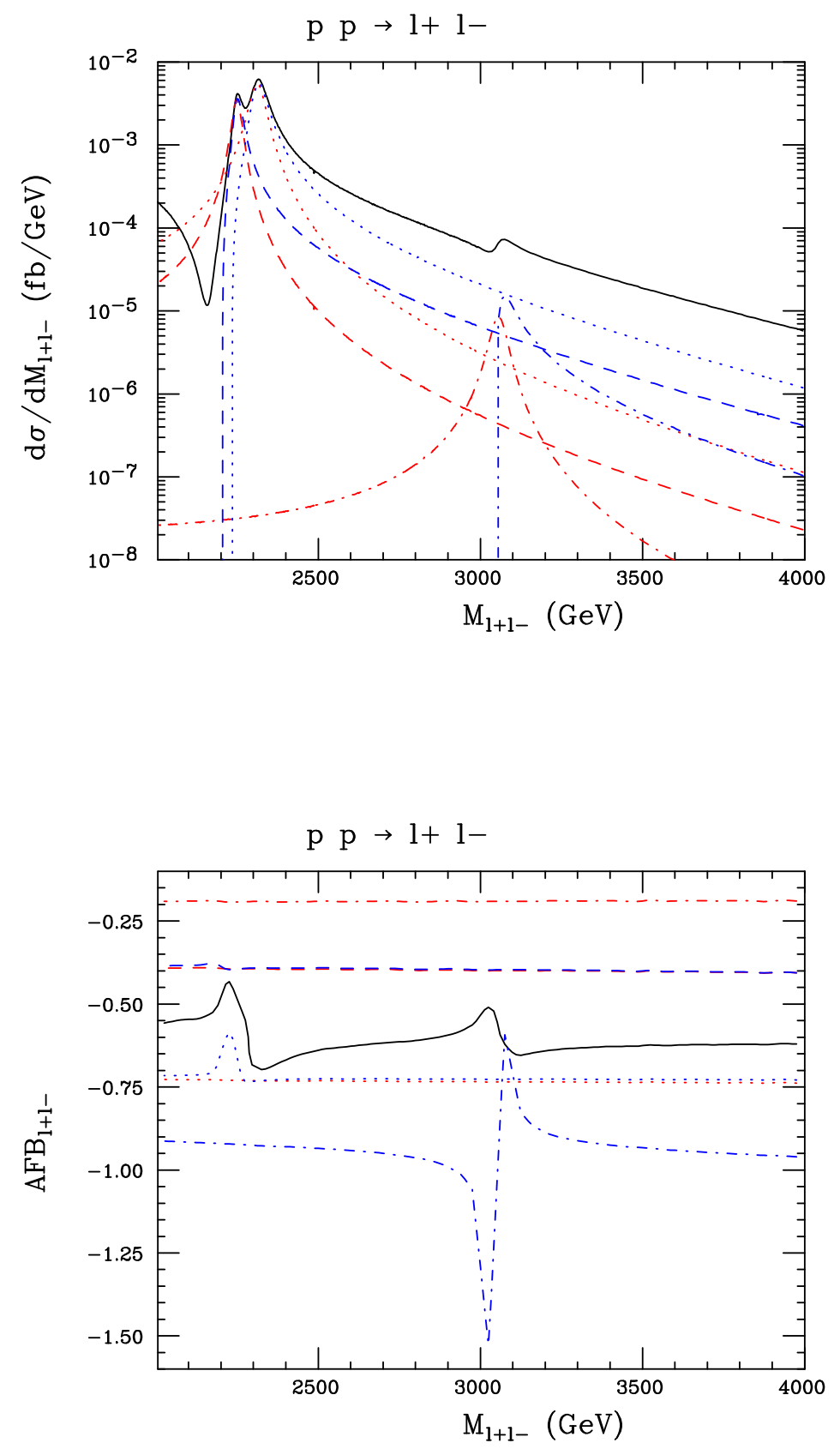

Figure 12: Differential distributions in invariant mass $M_{l^{+} l^{-}}$for (top) the cross section and (bottom) the forward-backward asymmetry at the $14 \mathrm{TeV}$ LHC for NC DY in the $4 \mathrm{DCHM}$ for $f=1.2 \mathrm{TeV}$ and $g^{*}=1.8$ in the case of the complete result (solid), for each resonance squared separately (dashed, dotted and dot-dashed in red) as well for their intereference with the SM plus the SM squared (dashed, dotted and dot-dashed in blue). Cuts, cross sections and mass/width parameters as in the previous plots. 

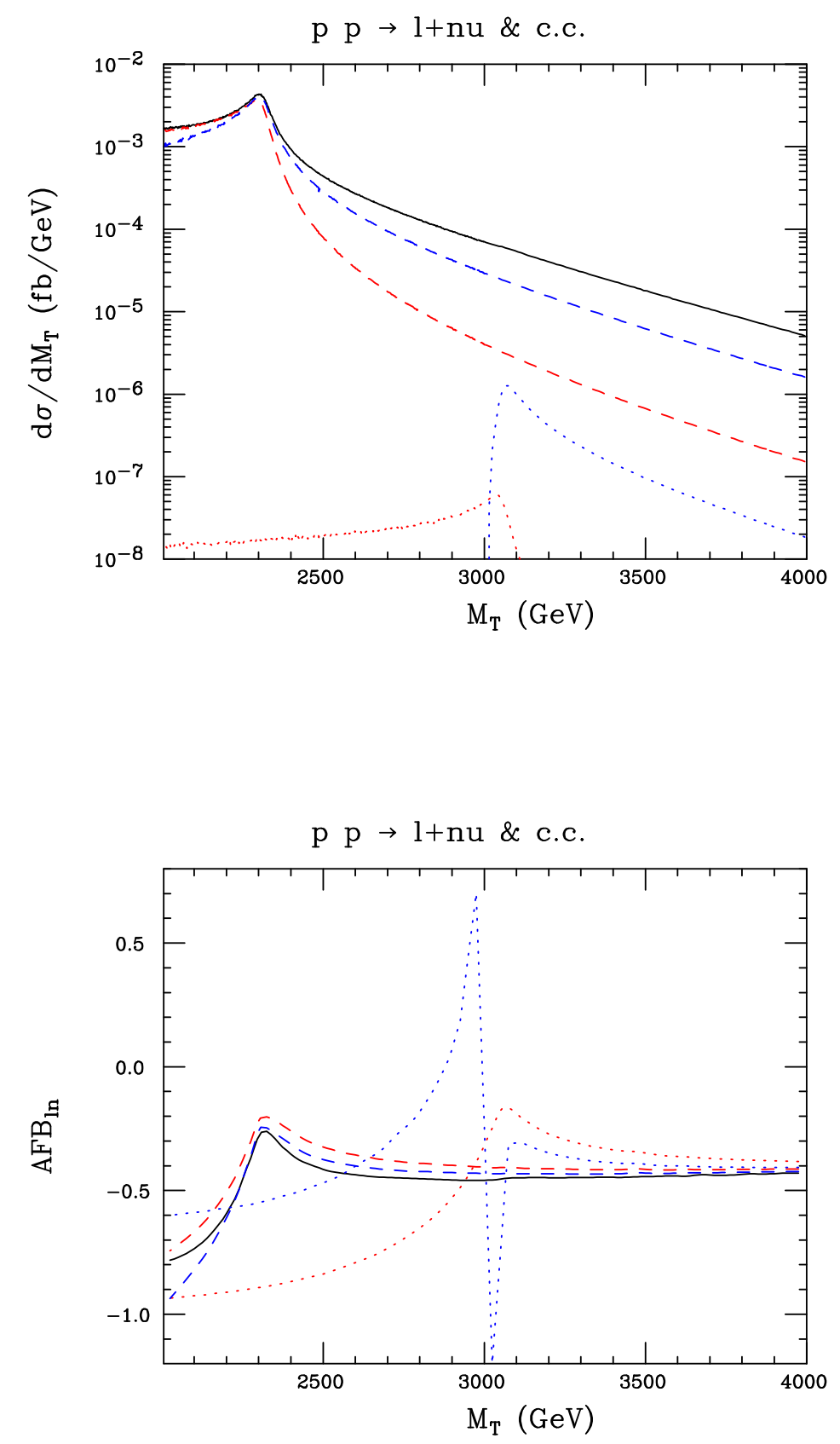

Figure 13: Differential distributions in transverse mass $M_{T}$ for (top) the cross section and (bottom) the forward-backward asymmetry at the 14 TeV LHC for CC DY in the $4 \mathrm{DCHM}$ for $f=1.2 \mathrm{TeV}$ and $g^{*}=1.8$ in the case of the complete result (solid), for each resonance squared separately (dashed and dotted in red) as well for their intereference with the SM plus the SM squared (dashed and dotted in blue). Cuts, cross sections and mass/width parameters as in the previous plots. 
that the above is not an issue in the case of the cross section, particular attention has to be given to the AFB distributions. Given that AFB is defined in terms of the number of events measured in some 'forward' $\left(N_{F}\right)$ and 'backward' $\left(N_{B}\right)$ directions, the statistical error is evaluated by propagating the Poisson error on each measured quantity (i.e., $\left.\delta N_{F(B)}=\sqrt{N_{F(B)}}\right)$. Per given integrated luminosity $\mathcal{L}$, the measured number of events will be $N_{F(B)}=\varepsilon \mathcal{L} \sigma_{F(B)}, \sigma_{F(B)}$ being the integrated or differential 'forward(backward)' cross section, yielding an uncertainty on AFB of

$$
\delta(\mathrm{AFB}) \equiv \delta\left(\frac{N_{F}-N_{B}}{N_{F}+N_{B}}\right)=\sqrt{\frac{4}{\mathcal{L} \varepsilon} \frac{\sigma_{F} \sigma_{B}}{\left(\sigma_{F}+\sigma_{B}\right)^{3}}} .
$$

Here, $\varepsilon$ corresponds to the assumed reconstruction efficiency of the $l^{+} l^{-}$and $l^{+} \nu+$ c.c. systems.

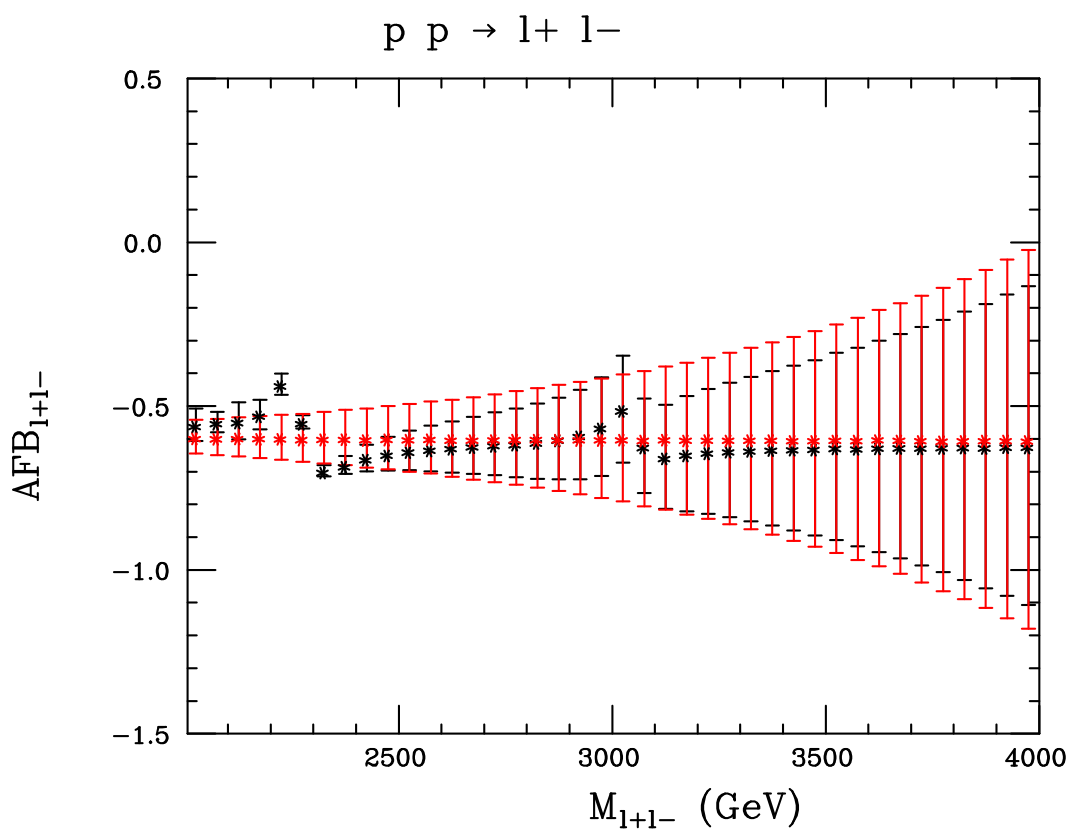

Figure 14: Differential distributions in invariant mass $M_{l^{+} l^{-}}$for the forward-backward asymmetry at the $14 \mathrm{TeV}$ LHC for NC DY in the 4DCHM for $f=1.2 \mathrm{TeV}$ and $g^{*}=1.8$, our benchmark (f), including error estimates, for $\mathcal{L}=1500 \mathrm{fb}^{-1}$ and $\varepsilon=90 \%$. Cuts, cross sections and mass/width parameters as in the previous plots. Bin width is here 50 GeV. Further, the $4 \mathrm{DCHM}(\mathrm{SM})$ is in black(red).

Figs. 1415 show our findings in this connection. Herein, the starred points are the central values for the given asymmetry, with a statistical error quantified by binning the cross sections in $M_{l^{+} l^{-}}\left(\mathrm{NC}\right.$ channel) and $M_{T}$ (CC channel), for an histogram width of $50 \mathrm{GeV}$. Assuming $\mathcal{L}=1500 \mathrm{fb}^{-1}$ and $\varepsilon=90 \%$, it is clear that 4 DCHM resonant effects in $\mathrm{AFB}$ can be discernible with respect to the $\mathrm{SM}$ noise in both the $\mathrm{NC}$ and $\mathrm{CC}$ case, albeit (probably) limitedly to the lowest lying resonances in either case, i.e., $Z_{2,3}$ and $W_{2}$, respectively, so long that very high luminosities can be attained (for example, at the Super-LHC). In the NC case, however, for di-electron final states, also the extraction of the heaviest neutral resonance $\left(Z_{5}\right)$ remains an open possibility. 


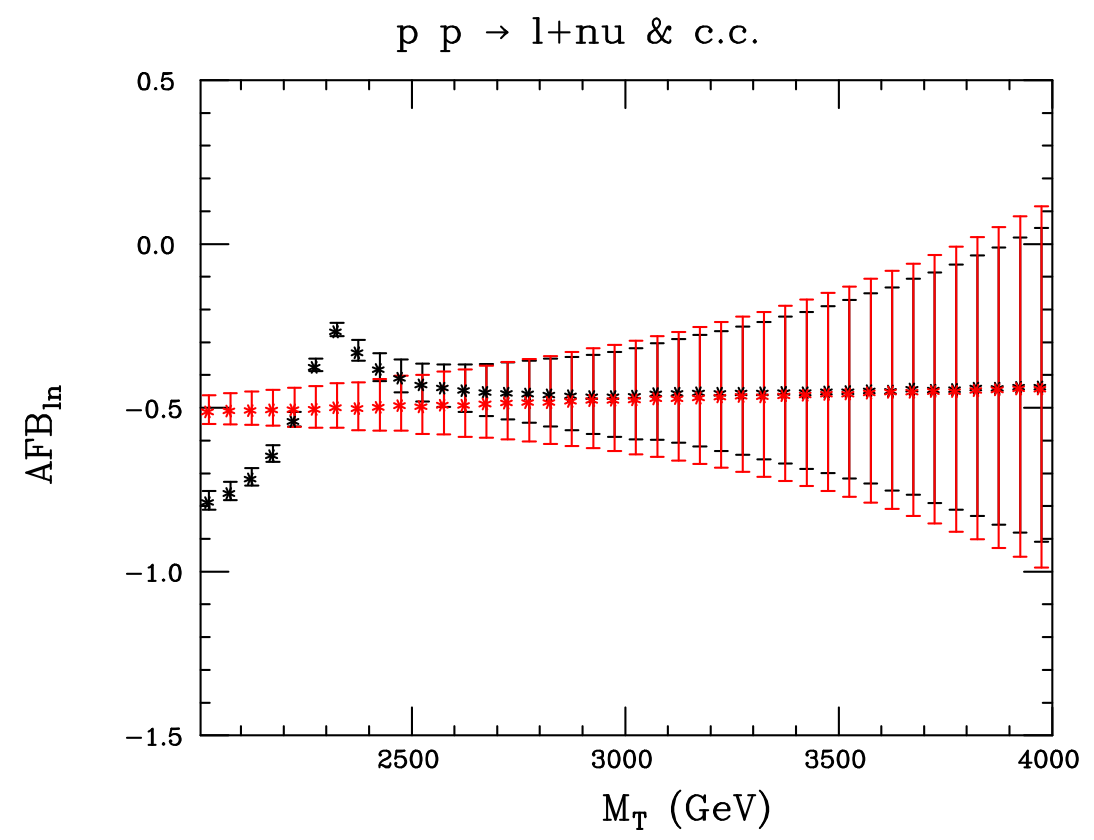

Figure 15: Differential distributions in transverse mass $M_{T}$ for the forward-backward asymmetry at the $14 \mathrm{TeV}$ LHC for CC DY in the 4DCHM for $f=1.2 \mathrm{TeV}$ and $g^{*}=1.8$, our benchmark (f), including error estimates, for $\mathcal{L}=1500 \mathrm{fb}^{-1}$ and $\varepsilon=90 \%$. Cuts, cross sections and mass/width parameters as in the previous plots. Bin width is here 50 GeV. Further, the $4 \mathrm{DCHM}(\mathrm{SM})$ is in black(red).

In view of such a high demand of luminosity to extract fully differential effects in AFB, it makes sense looking at integrated values of the latter, over suitable mass intervals. By inspecting Figs. 10 and 11, it is clear that differences between the 4DCHM and SM are maximised when one integrates to the left and to the right of each resonance. In the case of the NC channel, there are two mass points (in $M_{l^{+} l^{-}}$) where this can be attempted, again assuming a conservative $50 \mathrm{GeV}$ resolution, around the overlapping $Z_{2,3}$ resonances and then around $Z_{5}$. In the case of the $\mathrm{CC}$ channel, there is only one mass point (in $M_{T}$ ) to exploit for such a resolution, that is, around the $W_{2}$ resonance. (In essence, the extremes of each mass interval used in the integration procedure are defined by the crossing points between the 4DCHM and SM histograms in Figs. 10 and 11.)

Tab. 23 shows our findings, now for the complete list of benchmarks (a)-(f), assuming here a more modest luminosity, of $600 \mathrm{fb}^{-1}$, again with a $90 \%$ tagging efficiency. Depending on the benchmark at hand, it is clear that some resolving power between the 4DCHM and the SM exists for the integrated AFB, the more so the lighter the resonant masses involved, in both the $\mathrm{NC}$ and $\mathrm{CC}$ case. In fact, taking the difference between the integrate $\mathrm{AFB}$ values obtained to the left and to the right of each resonance seems the most effective way to disentangle the two models, as such a quantity is very nearly zero for the SM in all cases whereas it is not so (even in a statistical sense) for the 4DCHM.

If the mass difference between the $Z^{\prime}$ and $W^{\prime}$ gauge boson and the masses of the heavy fermion increase, a notable increase occurs in the width of the gauge bosons, as discussed in detail in sections 4.1.1a-4.1.1c. We study the impact of this phenomenon onto the mass distributions of both the NC and CC process in Figs. 16 and 17, where we keep the same gauge boson masses as till now (i.e., for the benchmark $f=1.2 \mathrm{TeV}$ 


\begin{tabular}{|c|c|c|}
\hline & (a) $f=0.75 \mathrm{TeV}$ and $g_{*}=2$ & \\
\hline & $\operatorname{AFB}\left(l^{+} l^{-}\right)$ & $\operatorname{AFB}\left(l^{+} \nu+\right.$ c.c. $)$ \\
\hline 4DCHM [I] & $(-0.554 \pm 0.013)[-0.627 \pm 0.029]-$ & $(-0.698 \pm 0.011)[-0.460 \pm 0.074]$ \\
\hline & $(-0.583 \pm 0.013)[-0.590 \pm 0.029]-$ & $(-0.533 \pm 0.011)[-0.478 \pm 0.074]$ \\
\hline 4DCHM [II] & $(-0.531 \pm 0.045)[-0.620 \pm 0.11]$ & \\
\hline SM & $(-0.593 \pm 0.045)[-0.598 \pm 0.11]$ & \\
\hline & (b) $f=0.8 \mathrm{TeV}$ and $g_{*}=2.5$ & \\
\hline 4DCHM [I] & $(-0.578 \pm 0.025)[-0.607 \pm 0.060]$ & $(-0.607 \pm 0.022)[-0.455 \pm 0.093]$ \\
\hline SM & $(-0.591 \pm 0.025)[-0.596 \pm 0.060]$ & $(-0.515 \pm 0.022)[-0.469 \pm 0.093]$ \\
\hline $4 \mathrm{DCHM}[\mathrm{II}]$ & $(-0.59 \pm 0.16)[-0.61 \pm 0.15]$ & \\
\hline $\mathrm{SM}$ & $(-0.60 \pm 0.16)[-0.60 \pm 0.15]$ & \\
\hline & (c) $f=1 \mathrm{TeV}$ and $g_{*}=2$ & \\
\hline 4DCHM [I] & $(-0.515 \pm 0.085)[-0.625 \pm 0.059]$ & $(-0.57 \pm 0.16)[-0.62 \pm 0.17]$ \\
\hline SM & $(-0.593 \pm 0.085)[-0.596 \pm 0.059]$ & $(-0.60 \pm 0.16)[-0.60 \pm 0.17]$ \\
\hline 4DCHM [II $]$ & $(-0.699 \pm 0.022)[-0.443 \pm 0.093]$ & \\
\hline SM & $(-0.514 \pm 0.022)[-0.469 \pm 0.093]$ & \\
\hline & (d) $f=1 \mathrm{TeV}$ and $g_{*}=2.5$ & \\
\hline 4DCHM [I] & $(-0.578 \pm 0.052)[-0.61 \pm 0.11]$ & $(-0.612 \pm 0.045)[-0.44 \pm 0.12]$ \\
\hline SM & $(-0.595 \pm 0.052)[-0.60 \pm 0.11]$ & $(-0.496 \pm 0.045)[-0.46 \pm 0.12]$ \\
\hline 4DCHM [II $]$ & $(-0.60 \pm 0.48)[-0.61 \pm 0.30]$ & \\
\hline $\mathrm{SM}$ & $(-0.60 \pm 0.48)[-0.60 \pm 0.30]$ & \\
\hline & (e) $f=1.1 \mathrm{TeV}$ and $g_{*}=1.8$ & \\
\hline 4DCHM [I] & $(-0.467 \pm 0.085)[-0.634 \pm 0.059]$ & $(-0.417 \pm 0.044)[-0.45 \pm 0.12]$ \\
\hline SM & $(-0.594 \pm 0.085)[-0.596 \pm 0.059]$ & $(-0.496 \pm 0.044)[-0.46 \pm 0.12]$ \\
\hline 4DCHM [II $]$ & $(-0.55 \pm 0.16)[-0.63 \pm 0.17]$ & \\
\hline SM & $(-0.60 \pm 0.16)[-0.60 \pm 0.17]$ & \\
\hline & (f) $f=1.2 \mathrm{TeV}$ and $g_{*}=1.8$ & \\
\hline 4DCHM [I] & $(-0.526 \pm 0.057)[-0.635 \pm 0.077]$ & $(-0.507 \pm 0.045)[-0.44 \pm 0.12]$ \\
\hline SM & $(-0.594 \pm 0.057)[-0.597 \pm 0.077]$ & $(-0.496 \pm 0.045)[-0.46 \pm 0.12]$ \\
\hline 4DCHM [II $]$ & $(-0.55 \pm 0.24)[-0.63 \pm 0.20]$ & \\
\hline SM & $(-0.60 \pm 0.24)[-0.60 \pm 0.20]$ & \\
\hline
\end{tabular}

Table 23: Value of the integrated AFB and relative error for the benchmarks of Tab. 20, obtained as described in the text, for the first [I] and (limited to the NC case) second resonance [II] in the mass interval to the (left)[right] of it, for both the $\mathrm{NC}\left(M_{l^{+} l^{-}}\right)$and CC $\left(M_{T}\right)$ channel, assuming $\mathcal{L}=600 \mathrm{fb}^{-1}$ and $\varepsilon=90 \%$.

and $g_{*}=1.8$ ), yet we gradually increase the widths of both the $Z^{\prime}$ and $W^{\prime}$ states, by selecting two further points in the low width regime and one each in the medium width regime and large width regime (as detailed in the captions).

As the values of $\Gamma_{Z^{\prime}}$ grow larger, the resolution of the separate $Z^{\prime}$ resonances, $Z_{2}$ and $Z_{3}$, is gradually no longer possible, as they start merging, with the ultimate tendency, for very large widths, to flatten down significantly, to the extent that the extraction of a narrow resonance (i.e., $\Gamma \ll M$ ) is no longer possible, rather the signal will appear as an 


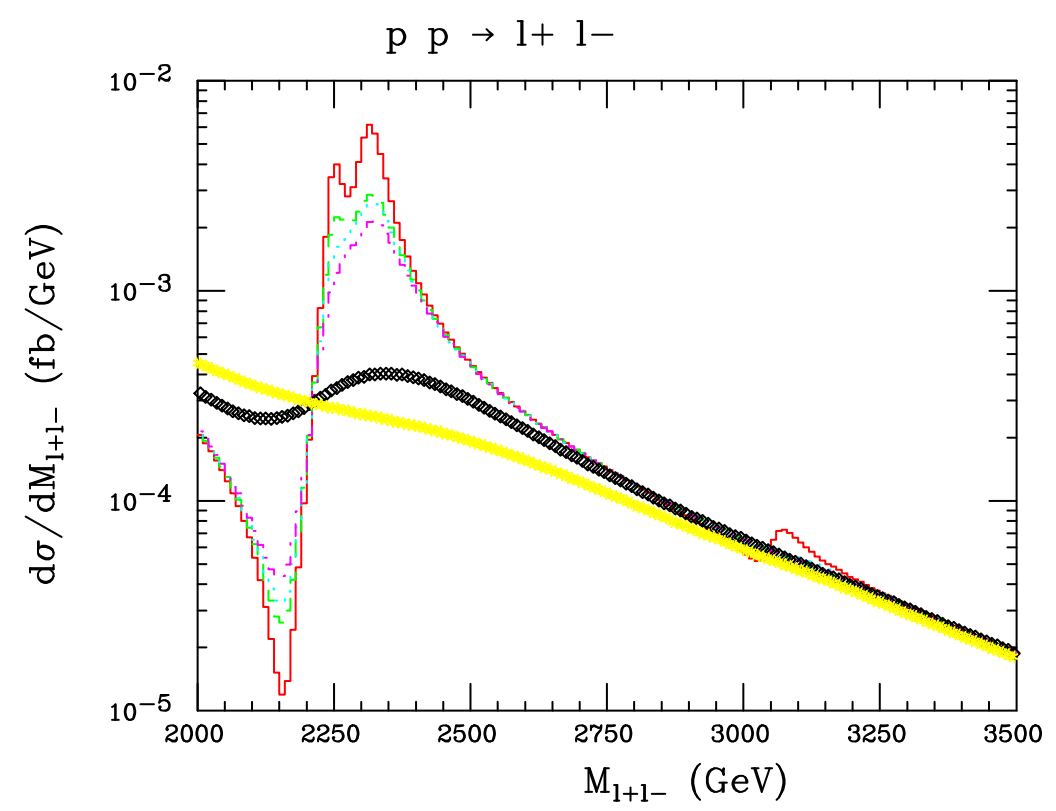

Figure 16: Differential distributions in invariant mass $M_{l^{+} l^{-}}$for the cross section at the $14 \mathrm{TeV}$ LHC for NC DY in the 4DCHM for $f=1.2 \mathrm{TeV}$ and $g^{*}=1.8$ for same mass and different width values of the resonant gauge bosons: the colour scheme is as in Fig. 5 . The integrated cross sections are $0.78,0.56,0.52,0.47,0.26$ and $0.23 \mathrm{fb}$, respectively. Cuts as in the previous plots. Bin width is here $10 \mathrm{GeV}$.

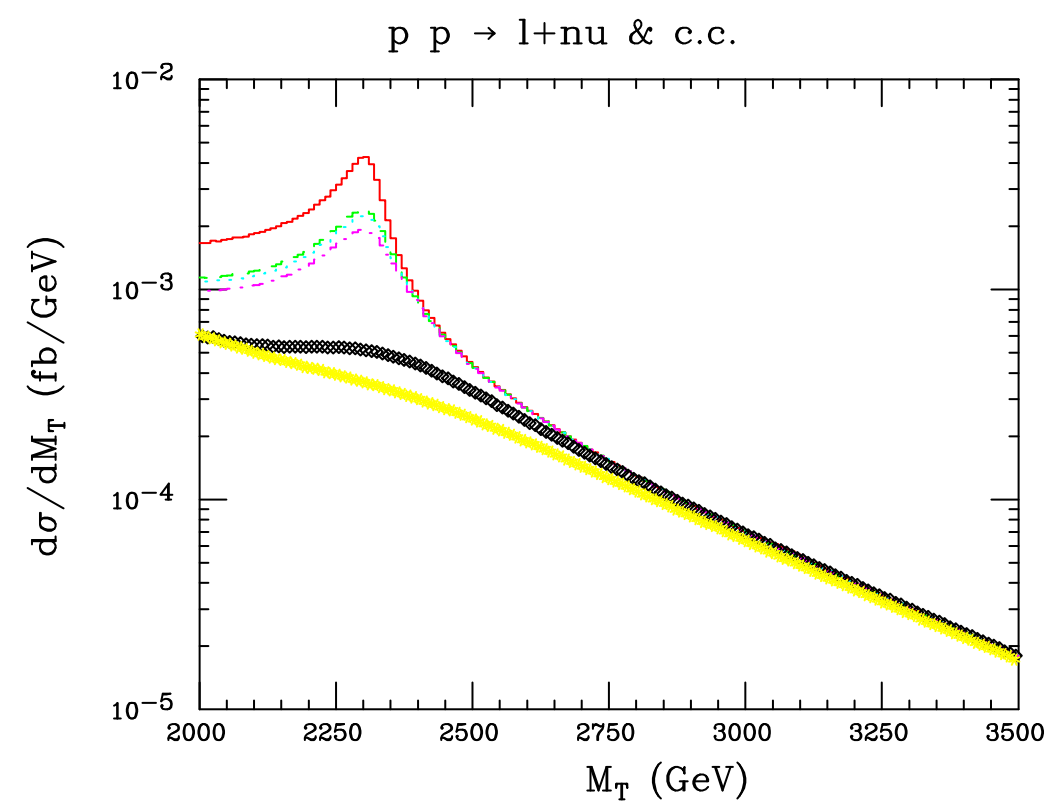

Figure 17: Differential distributions in transverse mass $M_{T}$ for the cross section at the $14 \mathrm{TeV}$ LHC for CC DY in the 4DCHM for $f=1.2 \mathrm{TeV}$ and $g^{*}=1.8$ for same mass and different width values of the resonant gauge bosons: the colour scheme is as in Fig. 5 . The integrated cross sections are 1.11, 0.79, 0.76, 0.70, 0.36 and $0.30 \mathrm{fb}$, respectively. Cuts as in the previous plots. Bin width is here $10 \mathrm{GeV}$. 
excess over a similarly shaped SM background. In the case of the heaviest resonance, the same phenomenology occur, though the non resonant limit is reached earlier on, owning to the fact that the original resonance only marginally emerged from the background. (Recall though that this state is hardly accessible at the LHC with standard design.) In the case of the only visible $W^{\prime}$ resonance $\left(W_{2}\right)$ the pattern is straightforward, the larger the value of $\Gamma_{W^{\prime}}$ the less prominent the mass peak, which essentially no longer stems out of the background as soon as one enters the medium width regime.

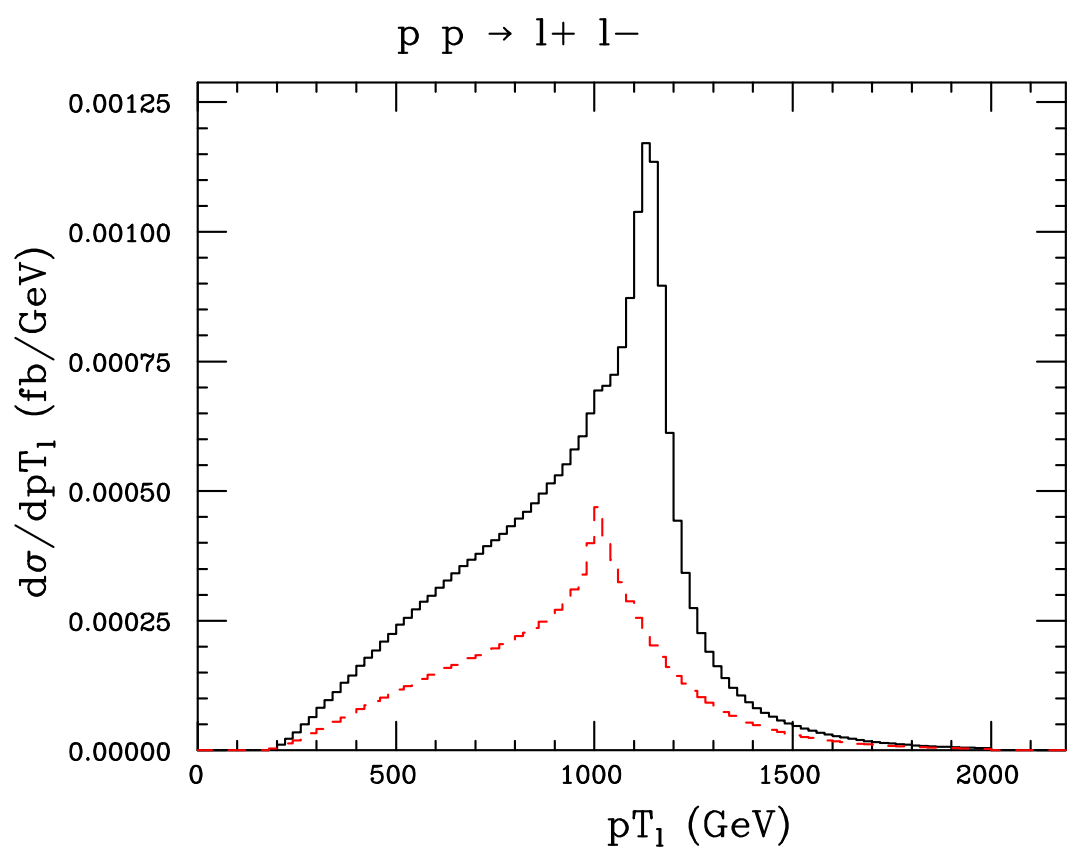

Figure 18: Differential distributions in transverse momentum $p_{l^{ \pm}}^{T}$ of the lepton for the cross section at the $14 \mathrm{TeV}$ LHC for NC DY in the 4DCHM for $f=1.2 \mathrm{TeV}$ and $g^{*}=1.8$ with $M_{Z_{2(3)[5]}}=2249(2312)[3056] \mathrm{GeV}$ and $\Gamma_{Z_{2(3)[5]}}=75(104)[313] \mathrm{GeV}$ (solid) plus the SM (dashed). Cuts as in the previous plots. Bin width is here $20 \mathrm{GeV}$.

One last exercise that we would like to perform before closing is to show how the exploitation of the fact that in the $4 \mathrm{DCHM}$ the mass of the two lightest $Z^{\prime}\left(Z_{2}\right.$ and $\left.Z_{3}\right)$ is strongly correlated to the one of the lightest $W^{\prime}$ state $\left(W_{2}\right)$, as previously explained. In fact, a similar correlation exists in the case of the heaviest states in the two sectors, neutral $\left(Z_{5}\right)$ and charged $\left(W_{3}\right)$, though this has no phenomenological relevance in the present context. As intimated, such a correlation can be exploited to improve searches in either channel. Assume for example that a $W^{\prime}$ resonance has been seen in the transverse mass spectrum and nothing appears instead in the invariant mass one, after the standard cuts in lepton and missing transverse momentum. This could well be justified by the fact that cross sections in the CC case are higher than in the NC one. Knowledge of $M_{W_{2}}$ would then imply also knowledge of $M_{Z_{2}}$ and $M_{Z_{3}}$, so that, to exalt the resonances associated to the latter, one may impose onto the di-lepton sample associated to the NC the cut, e.g., $p_{l}^{T}>M_{W_{2}} / 2$, extracted from analysis of the lepton-missing energy sample associated with the CC. (Recall that one can easily fit the $M_{W_{2}}$ value to the $M_{T}$ distribution using simulated data.). The advantage in doing so in order to enrich the candidate $Z^{\prime}$ sample of signal events is evident from Fig. 18, as most of the SM background would be eliminated 


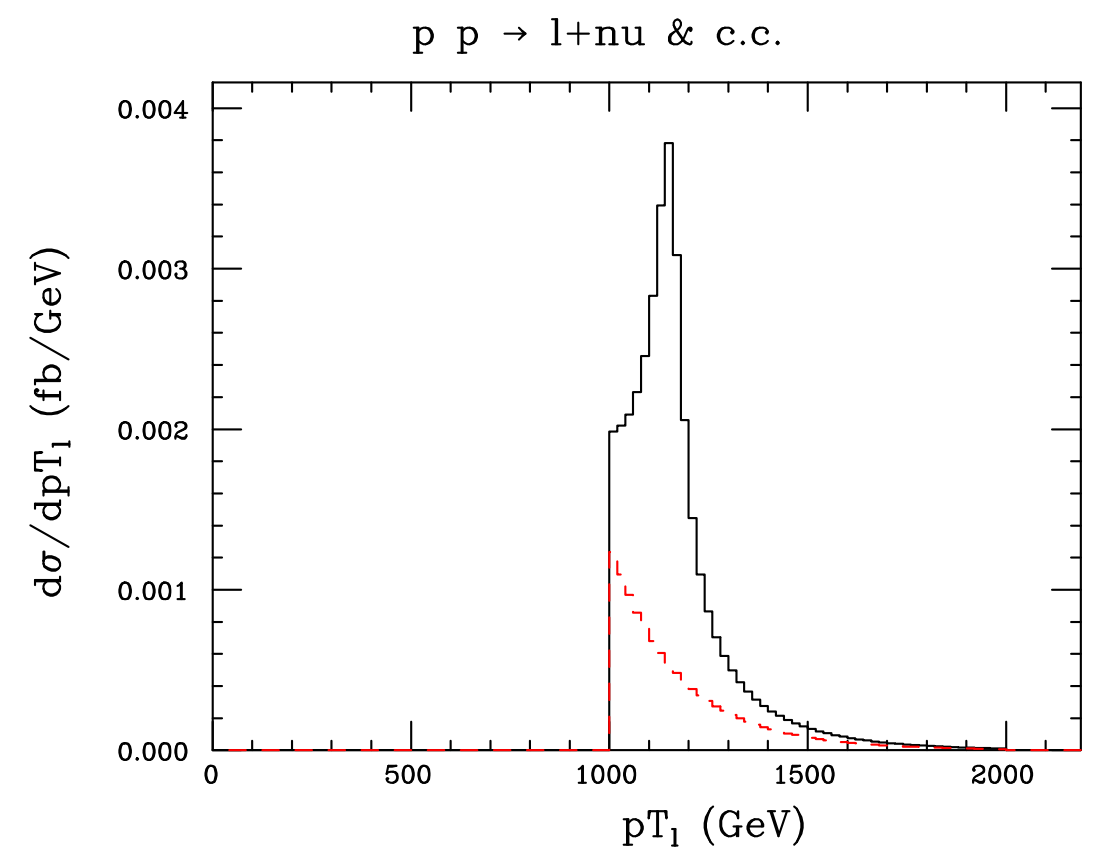

Figure 19: Differential distributions in transverse momentum $p_{l^{ \pm}}^{T}$ of the lepton for the cross section at the $14 \mathrm{TeV}$ LHC for CC DY in the 4DCHM for $f=1.2 \mathrm{TeV}$ and $g^{*}=1.8$ with $M_{W_{2(3)}}=2312.18(3056.1) \mathrm{GeV}$ and $\Gamma_{W_{2(3)}}=104(293) \mathrm{GeV}$ (solid) plus the SM (dashed). Cuts as in the previous plots. Bin width is here $20 \mathrm{GeV}$.

whereas the majority of the signal would be selected. The specular exercise would also be possible, as in the case of very narrow widths it could well be that it is a $Z^{\prime}$ signal the first to be seen. The benefit is evident here too, by investigating Fig. 19, Finally, notice that the $M_{T}$ and $p_{l}^{T}$ quantities are directly related in the CC case, unlike the NC one for $M_{l^{+} l^{-}}$and $p_{l}^{T}$, so that in the former case the lepton transverse momentum distribution displays a hard lower threshold, while this is not true in the latter case.

\section{Conclusions}

In summary, we have investigated the phenomenological implications of the 4DCHM for the case of DY processes at the LHC, in the case of both the NC and CC channels. By assuming only the 4DCHM configurations consistent with theoretical requirements of selfconsistency and current experimental limits, we have verified that only the $14 \mathrm{TeV}$ stage of the LHC has the potential to probe such a model (as the 7 and $8 \mathrm{TeV}$ data samples yield event rates below detectable level), assuming standard and Super-LHC luminosities, through both the aforementioned channels. The study of both the cross section and AFB distribution offers complementary handles to extract the underlying model spectrum in the gauge sector, although limited to the case of the lowest lying mass resonances, two in the case of the $\mathrm{NC}$ and one in the case of the CC.

Further, we have isolated a few salient features of the 4DCHM, with respect to popular models proposed in literature boasting the presence of $Z^{\prime}$ and/or $W^{\prime}$ bosons. Firstly, we have described how the opening of said decay channels impinges onto the line shape of the visible $Z^{\prime}$ and $W^{\prime}$ resonances in a manner that one can correlate the size of the 
width to the typical mass scale of the heavy fermions of the 4DCHM. Secondly, we have shown that almost degenerate $Z^{\prime}$ resonances appearing in the 4 DCHM spectrum can be resolved, so long that decays of the additional gauge bosons into pairs of heavy fermions are inaccessible. Thirdly, we have explained how to exploit the fact that $Z^{\prime}$ and $W^{\prime}$ masses are strongly correlated through the gauge structure of the 4DCHM in order to improve the extraction of the signals over the SM background, by cross-exchanging kinematic information between the $\mathrm{NC}$ and $\mathrm{CC}$ channel, and vice versa. In essence, we emphasise that it is the extraction of both the $Z_{2}$ and $Z_{3}$ resonances together with the $W_{2}$ one (either via cross section or asymmetry measurements), all rather close in mass, that would represent the hallmark signature of this model. Subsequently, an analysis of the new gauge boson line shapes would reveal, or otherwise, the presence of light additional fermions, shedding further light on the spectrum of this model.

This is the first phenomenological study of the 4DCHM for the case of the LHC, tackling the heavy gauge boson sector, limited to the case of DY production. However, the case of di-boson production is now under way too. Further studies are also ongoing regarding the heavy fermion and Higgs sectors. In fact, unlike more minimal versions of composite Higgs models, the 4DCHM suggests a realistic realisation of the common underslying dynamics of such scenarios which is fully testable at the LHC.

Instrumental to the accomplishement of the complete investigation of the $4 \mathrm{DCHM}$ at the CERN machine and as by-product of our study, we have produced and validated several computing modules, enabling the automated implementation and generation of the 4DCHM in the context of public tools such as LanHEP and CalcHEP. We have also generated a Mathematica code allowing one to perform said theoretical and experimental tests efficiently. Finally, the 4DCHM files are being uploaded onto the HEPMDB (https://hepmdb.soton.ac.uk/) for public use.

\section{Acknowledgments}

DB and SM are financed in part through the NExT Institute. The work of GMP has been supported by the German Research Foundation DFG through Grant No. STO876/21 and by BMBF Grant No. 05H09ODE, moreover he acknowledges financial support and hospitality by the University of Southampton. We would all like to thank M. Redi and A. Tesi for sharing with us the algorithm that computes the Higgs' VEV and the Higgs' mass and for continuous advice and encouragement. We finally thank also A. Semenov as well as A. Pukhov for the help given in solving some problems that we have found during the implementation of the model in LanHEP and/or CalcHEP. 


\section{A Appendix A: Analytical expressions}

\section{A.1 Masses of the gauge bosons}

At the leading order in $\xi=v^{2} / f^{2}$, where $v$ is the VEV of the Higgs, the analytical expressions of the neutral gauge boson masses are given by

$$
\begin{aligned}
& M_{\gamma}^{2}=0, \\
& M_{Z}^{2} \simeq \frac{f^{2}}{4} g_{*}^{2}\left(s_{\theta}^{2}+\frac{s_{\psi}^{2}}{2}\right) \xi, \\
& M_{Z_{1}}^{2}=f^{2} g_{*}^{2}, \\
& M_{Z_{2}}^{2} \simeq \frac{f^{2} g_{*}^{2}}{c_{\psi}^{2}}\left(1-\frac{s_{\psi}^{2} c_{\psi}^{4}}{4 c_{2 \psi}} \xi\right), \\
& M_{Z_{3}}^{2} \simeq \frac{f^{2} g_{*}^{2}}{c_{\theta}^{2}}\left(1-\frac{s_{\theta}^{2} c_{\theta}^{4}}{4 c_{2 \theta}} \xi\right), \\
& M_{Z_{4}}^{2}=2 f^{2} g_{*}^{2}, \\
& M_{Z_{5}}^{2} \simeq 2 f^{2} g_{*}^{2}\left(1+\frac{1}{16}\left(\frac{1}{c_{2 \theta}}+\frac{1}{2 c_{2 \psi}}\right) \xi\right)
\end{aligned}
$$

with $\tan \theta=\left(s_{\theta} / c_{\theta}\right)=\left(g_{0} / g_{*}\right)$ and $\tan \psi=\left(s_{\psi} / c_{\psi}\right)=\left(\sqrt{2} g_{0 Y} / g_{*}\right)$.

From these expressions we note that $Z_{1}$ and $Z_{4}$ have their masses completely determined by the composite sector and they do not receive any contribution from EWSB.

For the charged sector we have:

$$
\begin{aligned}
& M_{W}^{2} \simeq \frac{f^{2}}{4} g_{*}^{2} s_{\theta}^{2} \xi, \\
& M_{W_{1}}^{2}=f^{2} g_{*}^{2}, \\
& M_{W_{2}}^{2} \simeq \frac{f^{2} g_{*}^{2}}{c_{\theta}^{2}}\left(1-\frac{s_{\theta}^{2} c_{\theta}^{4}}{2 c_{2 \theta}} \xi\right), \\
& M_{W_{3}}^{2} \simeq 2 f^{2} g_{*}^{2}\left(1-\frac{s_{\theta}^{2}}{4 c_{2 \theta}} \xi\right) .
\end{aligned}
$$

In the same way as $Z_{1}$ and $Z_{4}$ do, also $W_{1}$ does not receive any mass correction from EWSB.

\section{A.2 Masses of the fermions}

The expressions of the top and bottom mass at the leading order in $\xi$ are given by

$$
\begin{aligned}
& m_{b}^{2} \simeq \xi \frac{m_{*}^{2}}{2} \frac{\tilde{\Delta}_{b_{L}}^{2} \tilde{\Delta}_{b_{R}}^{2} \tilde{Y}_{B}^{2}}{\left(1+F_{L}\right)} \\
& m_{t}^{2} \simeq \xi \frac{m_{*}^{2}}{2} \frac{\tilde{\Delta}_{t_{L}}^{2} \tilde{\Delta}_{t_{R}}^{2} \tilde{Y}_{T}^{2}}{\left(1+F_{L}\right)\left(1+F_{R}\right)},
\end{aligned}
$$

where we have defined $\tilde{\Delta}_{t_{L}, t_{R}, b_{L}, b_{R}}=\Delta_{t_{L}, t_{R}, b_{L}, b_{R}} / m_{*}, \tilde{Y}_{T, B}=Y_{T, B} / m_{*}, \tilde{M}_{Y_{T, B}}=M_{Y_{T, B}} / m_{*}$,

$$
F_{L}=\tilde{\Delta}_{t_{L}}^{2}\left(1+\tilde{M}_{Y_{T}}^{2}\right), \quad F_{R}=\tilde{\Delta}_{t_{R}}^{2}\left(1+\left(\tilde{M}_{Y_{T}}+\tilde{Y}_{T}\right)^{2}\right)
$$


and, for simplicity, we have taken $\Delta_{b_{L}}=\Delta_{b_{R}}=0$ except in the bottom mass expression.

At order $\xi=0$ the masses of the lightest fermionic resonances are given by

$$
\begin{aligned}
& m_{T_{1}}^{2} \simeq \frac{m_{*}^{2}}{2}\left(2+\tilde{M}_{Y_{T}}^{2}-\tilde{M}_{Y_{T}} \sqrt{4+\tilde{M}_{Y_{T}}^{2}}\right)=m_{\tilde{T}_{1}}^{2}, \quad\left|M_{Y_{T}}\right|>\left|M_{Y_{B}}\right| \\
& m_{B_{1}}^{2} \simeq \frac{m_{*}^{2}}{2}\left(2+\tilde{M}_{Y_{B}}^{2}-\tilde{M}_{Y_{B}} \sqrt{4+\tilde{M}_{Y_{B}}^{2}}\right)=m_{\tilde{B}_{1}}^{2},
\end{aligned}
$$

where again we have taken $\Delta_{b_{L}}=\Delta_{b_{R}}=0$.

\section{A.3 Couplings of the gauge bosons to the fermions}

We give here the analytical expressions of the couplings of both the neutral and charged gauge bosons to the three generations of leptons and the first two generations of quarks at the leading order in $\xi$.

Starting from the elementary sector, where the neutral gauge fields of $S U(2)_{L} \times U(1)_{Y}$ are coupled with the light fermionic currents, we get, after taking into account the mixing among the fields, the following expression for the neutral current Lagrangian:

$$
\mathcal{L}_{N C}=\sum_{f}\left[e \bar{\psi}^{f} \gamma_{\mu} Q^{f} \psi^{f} A^{\mu}+\sum_{i=0}^{5}\left(\bar{\psi}_{L}^{f} g_{Z_{i}}^{L}(f) \gamma_{\mu} \psi_{L}^{f}+\bar{\psi}_{R}^{f} g_{Z_{i}}^{R}(f) \gamma_{\mu} \psi_{R}^{f}\right) Z_{i}^{\mu}\right],
$$

where $\psi_{L, R}=\left[\left(1 \pm \gamma_{5}\right) / 2\right] \psi$ and the label $i=0$ corresponds to the neutral SM gauge boson $Z$. The photon field, $A_{\mu}$, is coupled to the electromagnetic current in the standard way, i.e., with

$$
e=\frac{g_{L} g_{Y}}{\sqrt{g_{L}^{2}+g_{Y}^{2}}}, \quad g_{L}=g_{0} c_{\theta}, \quad g_{Y}=g_{0 Y} c_{\psi}
$$

while the couplings of the $Z_{i}$ 's have the following expressions:

$$
g_{Z i}^{L}(f)=A_{Z_{i}} T_{L}^{3}(f)+B_{Z_{i}} Q^{f}, \quad g_{Z i}^{R}(f)=B_{Z_{i}} Q^{f},
$$

where $A_{Z_{i}}=\left(g_{0} \alpha_{i}-g_{0 Y} \beta_{i}\right), B_{Z_{i}}=g_{0 Y} \beta_{i}$, with $\alpha_{i}$ and $\beta_{i}$ being the diagonalisation matrix elements, namely:

$$
W_{3}=\sum_{i=0}^{5} \alpha_{i} Z_{i}, \quad Y=\sum_{i=0}^{5} \beta_{i} Z_{i} .
$$

Here $W_{3}$ and $Y$ are the elementary gauge field associated to $S U(2)_{L}$ and $U(1)_{Y}$, respectively. At the leading order in $\xi$ we get:

$$
\begin{aligned}
& A_{Z}=\sqrt{g_{L}^{2}+g_{Y}^{2}}\left[1+\left(\frac{g_{L}^{2}}{g_{L}^{2}+g_{Y}^{2}} a_{Z}+\frac{g_{Y}^{2}}{g_{L}^{2}+g_{Y}^{2}} b_{Z}\right) \xi\right], \quad B_{Z}=-\frac{g_{Y}^{2}}{\sqrt{g_{L}^{2}+g_{Y}^{2}}}\left(1+b_{Z} \xi\right)(28) \\
& A_{Z_{2}}=-g_{Y} \frac{s_{\psi}}{c_{\psi}}\left[1+\left(\frac{g_{L}}{g_{Y}} a_{Z_{2}}-b_{Z_{2}}\right) \xi\right], \quad B_{Z_{2}}=g_{Y} \frac{s_{\psi}}{c_{\psi}}\left[1-b_{Z_{2}} \xi\right] \\
& A_{Z_{3}}=-g_{L} \frac{s_{\theta}}{c_{\theta}}\left[1+\left(a_{Z_{3}}+\frac{g_{Y}}{g_{L}} b_{Z_{3}}\right) \xi\right], \quad B_{Z_{3}}=g_{Y} \frac{s_{\theta}}{c_{\theta}} b_{Z_{3}} \xi \\
& A_{Z_{5}}=\left(g_{L} a_{Z_{5}}-g_{Y} b_{Z_{5}}\right) \sqrt{\xi}, \quad B_{Z_{5}}=g_{Y} b_{Z_{5}} \sqrt{\xi}
\end{aligned}
$$


with

$$
\begin{aligned}
a_{Z} & =\quad\left(2 s_{\theta}^{2}+s_{\psi}^{2}\right)\left(4 c_{\theta}^{2}-1\right) / 32, \quad b_{Z}=\left(2 s_{\theta}^{2}+s_{\psi}^{2}\right)\left(4 c_{\psi}^{2}-1\right) / 32, \\
a_{Z_{2}} & =\frac{\sqrt{2} s_{\theta} s_{\psi} c_{\psi}^{6}}{4\left(c_{\psi}^{2}-c_{\theta}^{2}\right)\left(2 c_{\psi}^{2}-1\right)}, \quad b_{Z_{2}}=\frac{c_{\psi}^{4}\left(2-7 c_{\psi}^{2}+9 c_{\psi}^{4}-4 c_{\psi}^{6}\right)}{8 s_{\psi}^{2}\left(1-2 c_{\psi}^{2}\right)^{2}}, \\
a_{Z_{3}} & =\frac{-2 c_{\theta}^{4}+5 c_{\theta}^{6}-4 c_{\theta}^{8}}{4\left(1-2 c_{\theta}^{2}\right)^{2}}, \quad b_{Z_{3}}=\frac{\sqrt{2} s_{\theta} s_{\psi} c_{\theta}^{6}}{4\left(2 c_{\theta}^{2}-1\right)\left(c_{\theta}^{2}-c_{\psi}^{2}\right)}, \\
a_{Z_{5}} & =\frac{s_{\theta}}{2 \sqrt{2}\left(1-2 c_{\theta}^{2}\right)}, \quad b_{Z_{5}}=-\frac{s_{\psi}}{4\left(1-2 c_{\psi}^{2}\right)}
\end{aligned}
$$

In the same way we can work out the expressions for the charged sector that are:

$$
\mathcal{L}_{C C}=\left[g_{W}^{+} W^{+}+g_{W_{1}}^{+} W_{1}^{+}+g_{W_{2}}^{+} W_{2}^{+}+g_{W_{3}}^{+} W_{3}^{+}\right] J^{-}+\text {h.c. }
$$

with $J^{ \pm}=\left(J^{1} \pm i J^{2}\right) / 2, J_{\mu}^{i}=\bar{\psi} T_{L}^{i} \gamma_{\mu}\left[\left(1-\gamma_{5}\right) / 2\right] \psi$, and

$$
\begin{aligned}
g_{W}^{ \pm} & =-\frac{g_{*} s_{\theta}}{\sqrt{2}}\left(1+\frac{s_{\theta}}{4 c_{\theta}} a_{12} \xi\right) \\
g_{W_{1}}^{ \pm} & =0 \\
g_{W_{2}}^{ \pm} & =\frac{g_{*} s_{\theta}^{2}}{\sqrt{2} c_{\theta}}\left(1+\frac{1}{4}\left(a_{22}-\frac{c_{\theta}}{s_{\theta}} a_{12}\right) \xi\right) \\
g_{W_{3}}^{ \pm} & =\frac{g_{*} s_{\theta}^{2}}{2 \sqrt{2} c_{\theta}} a_{24} \sqrt{\xi}
\end{aligned}
$$

where

$$
a_{12}=-\frac{1}{4} c_{\theta}\left(1-4 c_{\theta}^{2}\right) s_{\theta}, \quad a_{22}=-\frac{c_{\theta}^{2}}{4\left(1-2 c_{\theta}^{2}\right)^{2}}, \quad a_{24}=-\frac{c_{\theta}}{\sqrt{2}\left(1-2 c_{\theta}^{2}\right)} .
$$

Because of the non-universality of the couplings of the gauge sectors to the three generations of quarks the expressions of the coupling of the neutral and charged gauge bosons to the top and the bottom quark are different with respect to the first two generations of quarks. As an example we show the couplings of the neutral gauge bosons to the top quark and, as the expressions turn out to be quite complicated even at the leading order in $\xi$, we only show the terms originating from the elementary-composite mixing before EWSB $(\xi=0)$. After taking into account the mixing among the gauge and fermionic fields the neutral current Lagrangian is the following:

$$
\mathcal{L}_{N C}^{t}=\frac{2}{3} e \bar{\psi}^{t} \gamma_{\mu} \psi^{t} A^{\mu}+\sum_{i=0}^{5}\left(g_{Z_{i}}^{L}(t) \bar{\psi}_{L}^{t} \gamma_{\mu} \psi_{L}^{t}+g_{Z_{i}}^{R}(t) \bar{\psi}_{R}^{t} \gamma^{\mu} \psi_{R}^{t}\right) Z_{i}^{\mu}
$$

where 


$$
\begin{array}{rlrl}
g_{Z_{0}}^{L}(t)=\frac{e}{s_{\omega} c_{\omega}}\left(\frac{1}{2}-\frac{2}{3} s_{\omega}^{2}\right)+\mathcal{O}(\xi), & g_{Z_{0}}^{R}(t)=\frac{e}{s_{\omega} c_{\omega}}\left(-\frac{2}{3} s_{\omega}^{2}\right)+\mathcal{O}(\xi), \\
g_{Z_{1}}^{L}(t) \sim \mathcal{O}(\xi), & g_{Z_{1}}^{R}(t) \sim \mathcal{O}(\xi) \\
g_{Z_{2}}^{L}(t)=\frac{e}{6 c_{\omega}} \frac{s_{\psi}}{c_{\psi}} \frac{1}{\left(1+F_{L}\right)}\left(1-\frac{c_{\psi}^{2}}{s_{\psi}^{2}} F_{L}\right)+\mathcal{O}(\xi), & g_{Z_{2}}^{R}(t)=\frac{2 e}{3 c_{\omega}} \frac{s_{\psi}}{c_{\psi}} \frac{1}{\left(1+F_{R}\right)}\left(1-\frac{c_{\psi}^{2}}{s_{\psi}^{2}} F_{R}\right)+\mathcal{O}(\xi), \\
g_{Z_{3}}^{L}(t)=-\frac{e}{2 s_{\omega}} \frac{s_{\theta}}{c_{\theta}} \frac{1}{\left(1+F_{L}\right)}\left(1-\frac{c_{\theta}^{2}}{s_{\theta}^{2}} F_{L}\right)+\mathcal{O}(\xi), & g_{Z_{3}}^{R}(t) \sim \mathcal{O}(\xi), \\
g_{Z_{4}}^{L}(t) \sim g_{Z_{4}}^{R}(t)=0, & g_{Z_{5}}^{L}(t) & \sim g_{Z_{5}}^{R}(t) \sim \mathcal{O}(\sqrt{\xi}),
\end{array}
$$

with

$$
\tan \omega=\frac{g_{Y}}{g_{L}}, \quad e=g_{L} s_{\omega}=g_{Y} c_{\omega}, \quad \frac{e}{s_{\omega} c_{\omega}}=\sqrt{g_{L}^{2}+g_{Y}^{2}} .
$$




\section{References}

[1] J. M. Campbell, J. Huston, and W. Stirling, Rept. Prog. Phys. 70, 89 (2007), arXiv:hep-ph/0611148.

[2] G. Cacciapaglia, C. Csaki, G. Marandella, and A. Strumia, Phys. Rev. D74, 033011 (2006), arXiv:hep-ph/0604111.

P. Langacker, Rev. Mod. Phys. 81, 1199 (2009), arXiv:hep-ph/0801.1345.

E. Salvioni, G. Villadoro, and F. Zwirner, JHEP 11, 068 (2009), arXiv:hep$\mathrm{ph} / 0909.1320$.

E. Accomando, A. Belyaev, L. Fedeli, S. F. King, and C. Shepherd-Themistocleous, Phys. Rev. D83, 075012 (2011), arXiv:1010.6058.

L. Basso, S. Moretti, and G. M. Pruna, JHEP 1108, 122 (2011), arXiv:1106.4762.

[3] P. Athron, S. King, D. Miller, S. Moretti, and R. Nevzorov, Phys. Rev. D84, 055006 (2011), arXiv:1102.4363.

P. Athron, S. King, D. Miller, S. Moretti, and R. Nevzorov, (2012), arXiv:1206.5028.

P. Athron, D. Stockinger, and A. Voigt, (2012), arXiv:1209.1470.

[4] L. Basso, B. O'Leary, W. Porod, and F. Staub, (2012), arXiv:1207.0507.

B. O'Leary, W. Porod, and F. Staub, JHEP 1205, 042 (2012), arXiv:1112.4600.

M. Hirsch, W. Porod, L. Reichert, and F. Staub, (2012), arXiv:1206.3516.

[5] M. Schmaltz and D. Tucker-Smith, Ann. Rev. Nucl. Part. Sci. 55, 229 (2005), arXiv:hep-ph/0502182.

M. Perelstein, Prog. Part. Nucl. Phys. 58, 247 (2007), arXiv:hep-ph/0512128.

H.-C. Cheng, (2007), arXiv:0710.3407.

[6] R. Contino, Y. Nomura, and A. Pomarol, Nucl. Phys. B671, 148 (2003), arXiv:hep$\mathrm{ph} / 0306259$.

[7] K. Agashe, R. Contino, and A. Pomarol, Nucl. Phys. B719, 165 (2005), arXiv:hep$\mathrm{ph} / 0412089$.

[8] R. Contino, (2010), arXiv:1005.4269.

[9] S. De Curtis, M. Redi, and A. Tesi, JHEP 1204, 042 (2012), arXiv:1110.1613.

[10] D. B. Kaplan and H. Georgi, Phys.Lett. B136, 183 (1984).

H. Georgi, D. B. Kaplan, and P. Galison, Phys.Lett. B143, 152 (1984).

H. Georgi and D. B. Kaplan, Phys.Lett. B145, 216 (1984).

M. J. Dugan, H. Georgi, and D. B. Kaplan, Nucl.Phys. B254, 299 (1985).

[11] ATLAS Collaboration, G. Aad et al., Phys. Lett. B716, 1 (2012), arXiv:1207.7214. 
[12] CMS Collaboration, S. Chatrchyan et al., Phys. Lett. B716, 30 (2012), arXiv:1207.7235.

[13] K. Agashe et al., Phys. Rev. D76, 115015 (2007), arXiv:0709.0007.

[14] K. Agashe, S. Gopalakrishna, T. Han, G.-Y. Huang, and A. Soni, Phys. Rev. D80, 075007 (2009), arXiv:0810.1497.

[15] G. Panico and A. Wulzer, JHEP 1109, 135 (2011), arXiv:1106.2719.

[16] R. Contino, T. Kramer, M. Son, and R. Sundrum, JHEP 0705, 074 (2007), arXiv:hep-ph/0612180.

[17] R. Contino, L. Da Rold, and A. Pomarol, Phys. Rev. D75, 055014 (2007), arXiv:hep$\mathrm{ph} / 0612048$.

[18] M. Redi and A. Tesi, (2012), arXiv:1205.0232.

[19] A. Semenov, (2010), arXiv:1005.1909.

[20] A. Pukhov et al., (1999), arXiv:hep-ph/9908288.

[21] A. Belyaev, N. D. Christensen, and A. Pukhov, (2012), arXiv:1207.6082.

[22] G. Belanger, N. D. Christensen, A. Pukhov, and A. Semenov, Comput. Phys. Commun. 182, 763 (2011), arXiv:1008.0181.

[23] M. Bondarenko et al., (2012), in arXiv:1203.1488.

[24] Mathematica Edition: Version 7.0 (Wolfram Research, Inc., Champaign, Illinois, 2008).

[25] CDF Collaboration, D0 Collaboration, T. Aaltonen et al., Phys. Rev. Lett. 109, 071804 (2012), arXiv:1207.6436.

[26] H. Murayama, I. Watanabe, and K. Hagiwara, HELAS: HELicity amplitude subroutines for Feynman diagram evaluations, 1992.

[27] T. Stelzer and W. Long, Comput. Phys. Commun. 81, 357 (1994), arXiv:hep$\mathrm{ph} / 9401258$.

[28] H. Kharraziha and S. Moretti, Comput. Phys. Commun. 127, 242 (2000), arXiv:hepph/9909313.

[29] R. Kleiss, W. J. Stirling, and S. Ellis, Comput. Phys. Commun. 40, 359 (1986).

[30] G. P. Lepage, J. Comput. Phys. 27, 192 (1978).

G. P. Lepage, VEGAS: AN ADAPTIVE MULTIDIMENSIONAL INTEGRATION PROGRAM, 1980. 
[31] N. E. Adam, V. Halyo, S. A. Yost, and W. Zhu, JHEP 0809, 133 (2008), arXiv:0808.0758.

N. E. Adam, V. Halyo, and S. A. Yost, JHEP 0805, 062 (2008), arXiv:0802.3251.

[32] G. Balossini et al., Acta Phys.Polon. B38, 3407 (2007).

[33] G. Balossini et al., JHEP 1001, 013 (2010), arXiv:0907.0276.

[34] CTEQ Collaboration, H. Lai et al., Eur. Phys. J. C12, 375 (2000), arXiv:hep$\mathrm{ph} / 9903282$.

[35] NNPDF Collaboration, R. D. Ball et al., Nucl.Phys. B855, 153 (2012), arXiv:1107.2652.

[36] ATLAS Collaboration, G. Aad et al., Phys. Lett. B701, 50 (2011), arXiv:1103.1391.

[37] CMS Collaboration, S. Chatrchyan et al., (2012), arXiv:1204.4764.

[38] ATLAS Collaboration, D. Hayden et al., EPJ Web Conf. 28, 12003 (2012), arXiv:1201.4721.

[39] CMS Collaboration, S. Chatrchyan et al., (2012), arXiv:1206.1849.

[40] CMS Collaboration, CMS-PAS-EXO-08-009 (2009).

[41] CMS Collaboration, CMS-PAS-EXO-09-012 (2009).

[42] ATLAS Collaboration, G. Aad et al., JHEP 1204, 069 (2012), arXiv:1202.5520.

[43] ATLAS Collaboration, G. Aad et al., Phys. Rev. Lett. 109, 032001 (2012), arXiv:1202.6540.

[44] D. Marzocca, M. Serone, and J. Shu, JHEP 1208, 013 (2012), arXiv:1205.0770.

[45] O. Matsedonskyi, G. Panico, and A. Wulzer, (2012), arXiv:1204.6333.

[46] F. Gianotti et al., Eur. Phys. J. C39, 293 (2005), arXiv:hep-ph/0204087.

[47] U. Baur, O. Brein, W. Hollik, C. Schappacher, and D. Wackeroth, Phys.Rev. D65, 033007 (2002), arXiv:hep-ph/0108274.

[48] J. C. Collins and D. E. Soper, Phys.Rev. D16, 2219 (1977). 